\title{
Twisted Flato-Fronsdal theorem for higher-spin algebras
}

\author{
Thomas Basile, ${ }^{a}$ Xavier Bekaert ${ }^{b}$ and Euihun Joung ${ }^{a}$ \\ ${ }^{a}$ Department of Physics and Research Institute of Basic Science, Kyung Hee University, \\ Seoul 02447, Korea \\ ${ }^{b}$ Institut Denis Poisson, Université de Tours, Université d'Orléans, CNRS, \\ Parc de Grandmont, 37200 Tours, France \\ E-mail: thomas.basile@khu.ac.kr, xavier.bekaert@lmpt.univ-tours.fr, \\ euihun. joung@khu.ac.kr
}

ABSTRACT: We explore the relation between the singleton and adjoint modules of higherspin algebras via $s o(2, d)$ characters. In order to relate the tensor product of the singleton and its dual to the adjoint module, we consider a heuristic formula involving symmetrization over the variables of the character. We show that our formula reproduces correctly the adjoint-module character for type-A (and its high-order extensions) and type-B higherspin gravity theories in any dimension. Implications and subtleties of this symmetrization prescription in other models are discussed.

KEYwords: Higher Spin Gravity, Higher Spin Symmetry

ARXIV EPRINT: 1802.03232 


\section{Contents}

1 Introduction 1

2 Four dimensions 3

3 General dimensions $\quad 11$

3.1 Type A 13

$\begin{array}{lll}3.2 & \text { Type B } & 15\end{array}$

3.3 Type $\mathrm{AB} \quad 20$

4 Lower dimensions $\quad 22$

4.1 Two dimensions 22

4.2 Three dimensions 26

5 Extensions and exceptions $\quad 33$

5.1 Type $\mathrm{A}_{\ell}$

$\begin{array}{lll}5.2 & \text { Type } \mathrm{B}_{\ell} & 34\end{array}$

5.3 Type $\mathrm{AB}_{\ell} \quad 36$

$\begin{array}{lll}5.4 & \text { Type } \mathrm{J} & 37\end{array}$

6 Discussion $\quad 41$

A Generalized Verma modules $\quad 42$

B Weyl character formula $\quad 43$

\section{Introduction}

In 1963, Dirac discovered two "remarkable representations" [1] of the isometry group of the four-dimensional anti de Sitter spacetime $\mathrm{AdS}_{4}$, which are the ultrashort modules of $\mathrm{SO}(2,3)$ nowadays known as the (Dirac) "singletons". Fifteen years later, Flato and Fronsdal showed that "one massless particle equals two Dirac singletons" [2], i.e. the tensor product of two singletons gives an infinite (direct) sum of massless particles of all integer spins. This result, often referred to as Flato-Fronsdal theorem, together with its generalizations (to supersymmetric and some higher-dimensional cases [3-6], to arbitrary dimensions $[7,8]$, to multilinetons $[9,10]$ as well as to arbitrary spin singletons [11]) has provided an important guiding principle for higher-spin gravity as it dictates consistent field contents of the theory prior to the actual construction of its dynamical equations.

Another cornerstone for higher-spin gravity theories is the higher-spin algebra playing the role of the global symmetry algebra associated to the gauge symmetry underlying the 
theory. Fradkin and Vasiliev first constructed a consistent higher-spin algebra [12], upon which the latter author obtained a set of nonlinear field equations describing interacting massless higher-spin fields propagating around $\mathrm{AdS}_{4}$ [13] (see e.g. [14, 15] for reviews of these equations and their higher-dimensional generalizations). Later on, it was realized that the higher-spin algebra can be viewed as the algebra of symmetries, namely the endomorphisms, of singletons [16]. This point of view allowed a wide range of generalizations, notably to dynamical equations for completely symmetric tensor gauge fields in higher dimensions [17].

To recapitulate, there are three key modules of the higher-spin algebra: the singleton (which plays a role analogous to the fundamental representation), the Hilbert space of the theory (the so-called "twisted-adjoint module") and finally the vector space of the higher-spin algebra itself (the "adjoint module"). The second module is the tensor product of the first one with itself, whereas the last module corresponds to the endomorphisms of the first one. In this sense, the adjoint module was identified in [18] with the tensor product of the singleton (denoted by Sng) with its dual (denoted by $\overline{\mathrm{Sng}}$ ). In [18], this naive relation between the tensor product module Sng $\otimes \overline{\text { Sng }}$ and the infinite sum of finitedimensional modules spanning the adjoint module was referred to as the "twisted FlatoFronsdal theorem". We will use here the same terminology for the refined relation that we will propose below.

A very convenient tool to handle various modules and their operations is the Lie algebra character. This mathematical object is closely related to the (one loop) partition function in physics (see e.g. [19-27] in the context of higher-spin holography) in the presence of chemical potentials for angular momenta. As partition functions contain most of physical information about the system under consideration, one can expect to be able to use characters in many mathematical analyses about the system. Indeed, the Flato-Fronsdal theorem was derived originally in a handy way using the $s o(2,3)$ character of Dirac singletons and those of massless spin- $s$ representations.

In this note, we reconsider the twisted Flato-Fronsdal theorem, that is, the relation

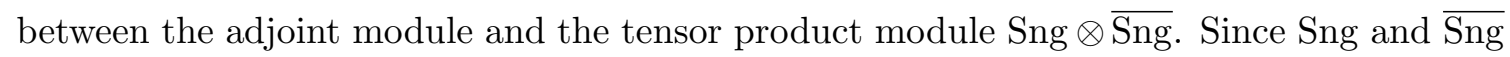
are respectively a lowest and a highest weight module of infinite dimension, the decomposition of their tensor product is subtle and requires a more careful treatment. In order to study this issue more concretely, we use the characters of the relevant modules and work with a prescription in which the characters can be manipulated in the usual manners. In this way, we find that the character of the tensor product module Sng $\otimes \overline{\text { Sng }}$ does not coincide with the character of the adjoint module. Analyzing in detail the four-dimensional massless higher-spin algebra, we find that the adjoint module differs from Sng $\otimes \overline{\text { Sng }}$ and obtain the precise relation between them by observing that the adjoint module character coincides in fact with the symmetrization of the Sng $\otimes \overline{\text { Sng }}$ character over the variables of the character. We examine this heuristic formula over other higher-spin algebras confirming its validity in the type- $\mathrm{A}_{\ell}$ and type-B theories in any dimensions, but mismatches by a few finite-dimensional modules in the type- $\mathrm{B}_{\ell \geqslant 2}$ and type- $\mathrm{J}$ cases. This confirms that the adjoint module is not given by the simple tensor product $\operatorname{Sng} \otimes \overline{\mathrm{Sng}}$, but requires proper amendments, about which we shall discuss throughout the paper. 
The organization of the paper is as follows. In the next section (section 2), we sketch the issue with the classical four-dimensional example. Then, we move to general dimensions in section 3. The cases of lower dimensions (two and three dimensions) are presented as useful toy models in section 4 . We extend our consideration to higher-order and higherspin singletons and find some exceptions to our conjecture in section 5. The last section contains a brief summary and discussion of our results. In appendix A, we collect some technical details on (generalized) Verma modules, while their Lie algebra characters (see e.g. [28-30] for the character formulae of all irreps of the conformal algebra) are discussed in appendix B.

\section{Four dimensions}

Let us first consider four-dimensional higher-spin gravity whose equations were initially constructed by Vasiliev in [13], and whose spectrum is concerned by Flato and Fronsdal's original result [2]. The four-dimensional Vasiliev equations contain an "interaction ambiguity" (first exhibited in [31] and further studied in e.g. [32-35]), given by a series of parameters. If the theory is required to have a definite parity, there remain only two allowed values for those parameters. These two choices are referred to as type A and type B, respectively. By now, it is understood that the type-A theory with the Neumann boundary condition $^{1}$ for the bulk scalar corresponds to the free scalar $\mathrm{CFT}_{3}[36]$, whereas the type-B theory with the Dirichlet condition corresponds to the free spinor $\mathrm{CFT}_{3}$ [37].

In the following, we shall review some details of the three modules of the higher-spin algebra - singleton, twisted-adjoint and adjoint modules. As they are also modules of the isometry subalgebra $s o(2,3)$, it will be useful to treat them using $s o(2,3)$ irreducible representations (irreps). For that, the basic object to consider is the lowest-weight module $\mathcal{V}(\Delta, s)$, whose character is given by

$$
\chi_{\mathcal{V}(\Delta, s)}^{s o(2,3)}(q, x)=\operatorname{Tr}_{\mathcal{V}(\Delta, s)}\left(q^{E} x^{J_{3}}\right)=\frac{q^{\Delta}}{(1-q)(1-q x)\left(1-q x^{-1}\right)} \chi_{s}^{s o(3)}(x),
$$

where the spin- $s$ character of $s o(3)$ is

$$
\chi_{s}^{s o(3)}(x):=\frac{x^{s+\frac{1}{2}}-x^{-s-\frac{1}{2}}}{x^{\frac{1}{2}}-x^{-\frac{1}{2}}} .
$$

Here $E$ and $J_{3}$ are the Cartan generators of $s o(2,3)$ (see appendix A where our conventions are summarized). For the purpose of the current section, it is sufficient to take the above formula for granted. Details about the derivation in any dimension will be provided in section 3. In terms of the variables $q=e^{-\beta}$ and $x=e^{i \alpha}$, this $s o(2,3)$ character reads

$$
\chi_{\mathcal{V}(\Delta, s)}^{s o(2,3)}(\beta, \alpha)=\frac{e^{-\left(\Delta-\frac{3}{2}\right) \beta}}{4 \sinh \frac{\beta}{2}(\cosh \beta-\cos \alpha)} \chi_{s}^{s o(3)}(\alpha), \quad \text { with } \quad \chi_{s}^{s o(3)}(\alpha)=\frac{\sin \left(s+\frac{1}{2}\right) \alpha}{\sin \frac{\alpha}{2}},
$$

and, the spin-s so(3) character coincides with the Dirichlet kernel.

\footnotetext{
${ }^{1}$ In the Poincaré patch of $\mathrm{AdS}_{4}$, the bulk scalar with Dirichlet condition approaches the boundary as $\phi(z, \vec{x}) \underset{z \rightarrow 0}{\sim} z \varphi(\vec{x})$ whereas the one with Neumann condition does as $\phi(z, \vec{x}) \underset{z \rightarrow 0}{\sim} z^{2} \varphi(\vec{x})$.
} 
Singleton module. The free massless scalar and spinor fields in three dimensions are nothing but the singleton representations that Dirac had found in [1]. Flato and Fronsdal named the latter and former as "Di" and "Rac", respectively. In terms of the lowest-weight module $\mathcal{V}(\Delta, s)$, the singletons Rac and Di correspond to the quotients,

$$
\operatorname{Rac}:=\mathcal{D}\left(\frac{1}{2}, 0\right)=\mathcal{V}\left(\frac{1}{2}, 0\right) / \mathcal{V}\left(\frac{5}{2}, 0\right), \quad \operatorname{Di}:=\mathcal{D}\left(1, \frac{1}{2}\right)=\mathcal{V}\left(1, \frac{1}{2}\right) / \mathcal{V}\left(2, \frac{1}{2}\right) .
$$

These representations are "ultrashort", even "minimal" in the sense (which can be made mathematically precise [38-40]) that they can be described as three-dimensional on-shell fields. Using the character $(2.3)$ of $\mathcal{V}(\Delta, s)$, it is simple to derive the characters of the singletons. They are

$$
\chi_{\operatorname{Rac}}^{s o(2,3)}(\beta, \alpha)=\frac{\cosh \frac{\beta}{2}}{\cosh \beta-\cos \alpha}, \quad \chi_{\mathrm{Di}}^{s o(2,3)}(\beta, \alpha)=\frac{\cos \frac{\alpha}{2}}{\cosh \beta-\cos \alpha} .
$$

It is also instructive to study the oscillator realization of the singletons. Using two sets of oscillators $\left(a, a^{\dagger}\right)$ and $\left(b, b^{\dagger}\right)$ with canonical commutation relations $\left[a, a^{\dagger}\right]=1=\left[b, b^{\dagger}\right]$, the generators of $s o(2,3)$ can be realized as [41-43]

$$
\begin{aligned}
E & =\frac{1}{2}\left(a^{\dagger} a+b^{\dagger} b+1\right), & J_{3} & =\frac{1}{2}\left(a^{\dagger} a-b^{\dagger} b\right), \\
J_{+} & =a^{\dagger} b, \quad L_{1}^{-}=\frac{1}{2}\left(a^{2}+b^{2}\right), & L_{2}^{-} & =-\frac{i}{2}\left(a^{2}-b^{2}\right), \quad L_{3}^{-}=a b,
\end{aligned}
$$

with $s o(3)=\operatorname{span}\left\{J_{+}, J_{-}, J_{3}\right\}$ and where $L_{a}^{-}(a=1,2,3)$ are the lowering operators with respect to $E$. The remaining generators are the Hermitian conjugates of the above (see appendix A for conventions). The Fock states,

$$
|m, n\rangle=\frac{\left(a^{\dagger}\right)^{m}\left(b^{\dagger}\right)^{n}}{\sqrt{m ! n !}}|0,0\rangle
$$

are eigenvectors of the Cartan subalgebra generators $E$ and $J_{3}$ :

$$
E|m, n\rangle=\frac{1}{2}(m+n+1)|m, n\rangle, \quad J_{3}|m, n\rangle=\frac{1}{2}(m-n)|m, n\rangle .
$$

The vacuum state $|0,0\rangle$ is the lowest-energy state of the Rac module, whereas the Di module has lowest-energy module spanned by the doublet $\{|1,0\rangle,|0,1\rangle\}$. Indeed, we have $\mathrm{Di}=\mathcal{D}\left(1, \frac{1}{2}\right)$, i.e. the vacuum carries a spin- $\frac{1}{2}$ representation of $s o(3)$ and its energy is one. The lowest-energy states of Rac and Di are annihilated by the lowering operators $L_{a}^{-}$. The full Rac and Di modules are then freely generated by applying the raising operators $L_{a}^{+}$. As a consequence Rac and Di are spanned by states $|m, n\rangle$ with even and odd $m+n$, respectively. Using these results, we can calculate the characters of the singletons as

$$
\chi_{\operatorname{Rac} / \mathrm{Di}}^{s o(2,3)}(\beta, \alpha)=\operatorname{Tr}_{\mathrm{Rac} / \mathrm{Di}}\left(e^{-\beta E+i \alpha J_{3}}\right)=\sum_{\text {even/odd } m+n} e^{-\beta \frac{m+n+1}{2}+i \alpha \frac{m-n}{2}} .
$$

To perform the sum, we can make the change of variables,

$$
m+n=2 s, \quad m-n=2(s-k),
$$


where $k=0,1, \ldots, 2 s$ and $s \in \mathbb{N}$ for Rac and $s \in \mathbb{N}+\frac{1}{2}$ for Di. Then, we get

$$
\chi_{\operatorname{Rac} / \mathrm{Di}}^{s o(2,3)}(\beta, \alpha)=\sum_{s \in \mathbb{N}+0 / \frac{1}{2}} e^{-\beta\left(s+\frac{1}{2}\right)} \sum_{k=0}^{2 s} e^{i \alpha(s-k)}=\sum_{s \in \mathbb{N}+0 / \frac{1}{2}} e^{-\beta\left(s+\frac{1}{2}\right)} \chi_{s}^{s o(3)}(\alpha) .
$$

The infinite sum in (2.11) leads to geometric series and one finally recovers the characters (2.5).

Twisted-adjoint module. All the other irreps $\mathcal{D}(\Delta, s)$ of $s o(2,3)$ in the unitary region $\Delta \geqslant s+1$ are much "longer" and they can be viewed as the Hilbert space of a fourdimensional on-shell field. In particular, the representations describing massless spin- $s$ particles on $\mathrm{AdS}_{4}$ lie at the unitary bound, and correspond to the quotients,

$$
\mathcal{D}(s+1, s)=\mathcal{V}(s+1, s) / \mathcal{V}(s+2, s-1),
$$

with the characters,

$$
\chi_{\mathcal{D}(s+1, s)}^{s o(2,3)}(\beta, \alpha)=\frac{e^{-\left(s-\frac{1}{2}\right) \beta} \sin \left(s+\frac{1}{2}\right) \alpha-e^{-\left(s+\frac{1}{2}\right) \beta} \sin \left(s-\frac{1}{2}\right) \alpha}{4 \sinh \frac{\beta}{2} \sin \frac{\alpha}{2}(\cosh \beta-\cos \alpha)} .
$$

Flato and Fronsdal have shown in [2] the following rule for the decomposition in irreducible so $(2,3)$-modules of the tensor product of two Rac or Di:

$$
\operatorname{Rac} \otimes \operatorname{Rac}=\bigoplus_{s=0}^{\infty} \mathcal{D}(s+1, s), \quad \operatorname{Di} \otimes \mathrm{Di}=\mathcal{D}(2,0) \oplus \bigoplus_{s=1}^{\infty} \mathcal{D}(s+1, s) .
$$

The right-hand-side of the above equations is nothing but the field content - namely, the twisted-adjoint module - of the type- $\mathrm{A}$ and type- $\mathrm{B}$ higher-spin gravity theories, respectively. This suggests that the $\mathrm{CFT}_{3}$ operators bilinear in the free massless scalar (Rac) or spinor (Di) fields - hence fall in the tensor product of two singletons representations - corresponds to the $\mathrm{AdS}_{4}$ massless gauge fields of higher-spin gravity together with one bulk scalar field (with "Neumann" or "Dirichlet" boundary conditions, respectively) $[36,37,44,45]$. This tensor product decomposition has been proven with the help of the $s o(2,3)$ characters by checking the following algebraic identities,

$$
\left(\chi_{\operatorname{Rac}}^{s o(2,3)}\right)^{2}=\sum_{s=0}^{\infty} \chi_{\mathcal{D}(s+1, s)}^{s o(2,3)}, \quad\left(\chi_{\mathrm{Di}}^{s o(2,3)}\right)^{2}=\chi_{\mathcal{D}(2,0)}+\sum_{s=1}^{\infty} \chi_{\mathcal{D}(s+1, s)}^{s o(2,3)} .
$$

In terms of oscillators, the tensor product of two singletons is realized by doubling the oscillators: $\left(a_{i}, a_{i}^{\dagger}\right)$ and $\left(b_{i}, b_{i}^{\dagger}\right)$ with $i=1,2$. Hence, the twisted-adjoint module is spanned by the states of the type,

$$
|m, n ; p, q\rangle=\frac{\left(a_{1}^{\dagger}\right)^{m}\left(b_{1}^{\dagger}\right)^{n}\left(a_{2}^{\dagger}\right)^{p}\left(b_{2}^{\dagger}\right)^{q}}{\sqrt{m ! n ! p ! q !}}|0,0 ; 0,0\rangle .
$$

Defining the action of an $s o(2,3)$ element $X$ on the singleton Fock state $|m, n\rangle$ as

$$
X|m, n\rangle=\sum_{p, q} R_{p, q}^{m, n}(X)|p, q\rangle,
$$


the action of $X$ on $|m, n ; p, q\rangle$ gives

$$
X|m, n ; p, q\rangle=\sum_{s, t}\left(R_{s, t}^{m, n}(X)|s, t ; p, q\rangle+R_{s, t}^{p, q}(X)|m, n ; s, t\rangle\right) .
$$

For the decomposition of the twisted-adjoint module into so(2,3)-irreducible ones, one can examine the lowest-weight states - that are annihilated by $L_{1}^{-}+i L_{2}^{-}$and $J_{+}$(then, consequently all $L_{a}^{-}$with $a=1,2,3$ annihilate the state) - in this doubled singleton Fock space (aka "doubleton"),

$$
\left(a_{1}^{2}+a_{2}^{2}\right)|\Psi\rangle=0=\left(a_{1}^{\dagger} b_{1}+a_{2}^{\dagger} b_{2}\right)|\Psi\rangle, \quad|\Psi\rangle=\sum_{m, n, p, q} c_{m, n, p, q}|m, n ; p, q\rangle .
$$

It is simple (see e.g. $[25,46,47])$ to show that any such a state $|\Psi\rangle$ is a linear combination of the lowest-weight states of $\mathcal{D}(s+1, s)$ (and $\mathcal{D}(2,0)$ for the case of $\mathrm{Di}$ ) hence confirming the rule (2.14).

Adjoint module. The adjoint module, namely the higher-spin algebra, is spanned by the higher-spin Killing tensors. For a given spin $s$, the Killing tensor is a finite-dimensional module of $s o(2,3)$. In terms of Young diagram, it corresponds to the rectangle made of two rows of length $s-1$,

$$
\begin{array}{|c|}
\hline s-1 \\
\hline s-1 \\
\hline
\end{array}
$$

whereas in terms of the lowest-weight module it corresponds to the non-unitary module $\mathcal{D}(1-s, s-1)$ defined by the following sequence of quotients,

$$
\begin{aligned}
\mathcal{D}(1-s, s-1) & =\mathcal{V}(1-s, s-1) / \mathcal{D}(2-s, s), \\
\mathcal{D}(2-s, s) & =\mathcal{V}(2-s, s) / \mathcal{D}(s+1, s),
\end{aligned}
$$

where $\mathcal{D}(s+1, s)$ is defined in (2.12). Here we used the Bernstein-Gel'fand-Gel'fand resolution detailed in [48]. Another point of view on this module makes use of the fact that it is finite-dimensional. The two real Lie algebras $s o(2,3)$ and $s o(5)$ are two distinct real forms of the same complex Lie algebra $s o_{\mathbb{C}}(5)$. The character of the finite-dimensional so(5)-module labeled by the dominant integral weight $(s-1, s-1)$ reads

$$
\chi_{(s-1, s-1)}^{s o(5)}\left(\alpha_{1}, \alpha_{2}\right)=\frac{\sin \left[\left(s-\frac{1}{2}\right) \alpha_{1}\right] \sin \left[\left(s+\frac{1}{2}\right) \alpha_{2}\right]-\sin \left[\left(s-\frac{1}{2}\right) \alpha_{2}\right] \sin \left[\left(s+\frac{1}{2}\right) \alpha_{1}\right]}{2 \sin \frac{\alpha_{1}}{2} \sin \frac{\alpha_{2}}{2}\left(\cos \alpha_{1}-\cos \alpha_{2}\right)} .
$$

Using the above information, we can obtain the corresponding so $(2,3)$ character, which is in fact simply related to the $s o(5)$ character $(2.22)$ as

$$
\chi_{\mathcal{D}(1-s, s-1)}^{s o(2,3)}(\beta, \alpha)=\chi_{(s-1, s-1)}^{s o(5)}(i \beta, \alpha) .
$$

Collecting all these results, we can calculate the so $(2,3)$ character of the adjoint module of the higher-spin algebra as

$$
\chi_{\text {Adj }}^{s o(2,3)}(\beta, \alpha)=\sum_{s=1}^{\infty} \chi_{\mathcal{D}(1-s, s-1)}^{s o(2,3)}(\beta, \alpha) .
$$


This infinite sum of characters involves a trigonometric series which is not convergent in the classical sense, but which is convergent in the sense of distribution theory. ${ }^{2}$ Accordingly, it can be evaluated using resummation techniques, ${ }^{3}$

$$
\sum_{n=1}^{\infty} \sin (n x)=\frac{1}{2} \cot (x / 2) .
$$

Using this formula, we obtain the character of the adjoint module as

$$
\chi_{\mathrm{Adj}}^{s o(2,3)}(\beta, \alpha)=\frac{\cosh ^{2} \frac{\beta}{2}+\cos ^{2} \frac{\alpha}{2}}{(\cosh \beta-\cos \alpha)^{2}} .
$$

Now the question is whether we can obtain the above character from the characters of the singletons (2.5). If this was possible in general, for an unknown higher-spin theory dual to a certain CFT with given spectrum, then we would be able to systematically identify the corresponding higher-spin algebra.

One of the simplest descriptions of the higher-spin algebra is viewing it as the algebra of endomorphisms of the singleton module,

$$
\operatorname{Adj}=\operatorname{End}(\text { Sng }),
$$

where "Sng" stands for either the Di or Rac module. We already know that the higher-spin algebra is identical both in type-A and type-B theories. Let us explore this point in the oscillator realization. Since the singleton module is the Fock space spanned by $|m, n\rangle$ (2.7), its endomorphism algebra can be generated by the operators,

$$
X^{m, n ; p, q}=\frac{\left(a^{\dagger}\right)^{m}\left(b^{\dagger}\right)^{n} a^{p} b^{q}}{\sqrt{m ! n ! p ! q !}}
$$

with even $m+n+p+q$. The above presentation of the higher-spin algebra is simply related to the more typical realization in terms of the oscillators $y_{\alpha}$ and $\bar{y}_{\dot{\alpha}},{ }^{4}$ by

$$
y_{1}=a+b^{\dagger}, \quad y_{2}=i\left(a^{\dagger}-b\right), \quad \bar{y}_{\dot{\alpha}}=\left(y_{\alpha}\right)^{\dagger} .
$$

The action of an $s o(2,3)$ element $X$ on this state is, by definition of the adjoint representation,

$$
X \triangleright X^{m, n ; p, q}=\left[X, X^{m, n ; p, q}\right] .
$$

However, this cannot be written easily in terms of the singleton representation $R_{m, n}^{p, q}(X)$. What is more naturally connected to the singleton representation is the basis,

$$
T^{m, n ; p, q}=|m, n\rangle\left\langle p, q\left|=\frac{\left(a^{\dagger}\right)^{m}\left(b^{\dagger}\right)^{n}}{\sqrt{m ! n !}}\right| 0,0\right\rangle\langle 0,0| \frac{a^{p} b^{q}}{\sqrt{p ! q !}},
$$

\footnotetext{
${ }^{2}$ See e.g. the section 6.13 of the book [49], devoted to the summability of Fourier series of periodic distributions.

${ }^{3}$ More precisely, this trigonometric series is Cesaro (thus Abel) resummable. For a proof of (2.25), see e.g. [50] (chapter XIII, section 60, Ex. 5).

${ }^{4}$ The higher-spin algebra is the algebra of even functions of $y_{\alpha}$ and $\bar{y}_{\dot{\alpha}}$ endowed with the Moyal star product.
} 
on which an $s o(2,3)$ element $X$ acts as

$$
X \triangleright T^{m, n ; p, q}=\sum_{s, t} R_{s, t}^{m, n}(X) T^{s, t ; p, q}-\overline{R^{p, q}\left(X^{\dagger}\right)} T^{m, n ; s, t} .
$$

Hence, in this basis, it becomes clear that the adjoint module is the tensor product of the singleton module - represented by $R_{s, t}^{m, n}(X)$ - and its dual module - represented by $-\overline{R_{s, t}^{p, q}\left(X^{\dagger}\right)}$. In order to relate $T^{m, n ; p, q}$ to the more standard basis $X^{m, n ; p, q}$, we need to realize the vacuum projector $|0,0\rangle\langle 0,0|$ as a function of oscillators,

$$
|0,0\rangle\langle 0,0|=\Pi_{\mathrm{vac}}\left(a, a^{\dagger}\right) \Pi_{\mathrm{vac}}\left(b, b^{\dagger}\right) .
$$

By imposing the conditions,

$$
\Pi_{\mathrm{vac}}^{\dagger}=\Pi_{\mathrm{vac}}, \quad \Pi_{\mathrm{vac}} a^{\dagger}=0, \quad \Pi_{\mathrm{vac}}^{2}=\Pi_{\mathrm{vac}},
$$

one can determine it as

$$
\Pi_{\mathrm{vac}}\left(a, a^{\dagger}\right)=\sum_{n=0}^{\infty} \frac{(-1)^{n}}{n !}\left(a^{\dagger}\right)^{n} a^{n} .
$$

Therefore, the $T^{m, n ; p, q}$ basis is related to the $X^{m, n ; p, q}$ basis as an infinite linear combination,

$$
T^{m, n ; p, q}=\sum_{s, t=0}^{\infty} \frac{(-1)^{s+t}}{\sqrt{C_{s}^{m+s} C_{s}^{p+s} C_{t}^{n+t} C_{t}^{q+t}}} X^{m+s, n+t ; p+s, q+t},
$$

where $C_{n}^{m}$ is the binomial coefficient. If we restrict the higher-spin algebra to all finite linear combinations of $X^{m, n ; p, q}$ - hence polynomials in the oscillators - then the basis $T^{m, n ; p, q}$ does not belong to the higher-spin algebra. In other words, the finite linear combinations of $X^{m, n ; p, q}$ and $T^{m, n ; p, q}$ give two distinct endomorphism algebras. This subtlety arises due to the fact that we are dealing with infinite-dimensional spaces.

Having this subtlety in mind, let us proceed further. From the viewpoint of the endomorphisms in the $T^{m, n ; p, q}$ basis, one would expect the adjoint module to be the tensor product of a singleton and its anti-singleton (as first pointed out in [18]):

$$
\operatorname{Adj} \stackrel{?}{=} \operatorname{Sng} \otimes \overline{\operatorname{Sng}},
$$

where we put the question mark at the equality because of an inconsistency we shall face soon below. The anti-singleton, denoted by "Sng", is a highest-weight module with maximal energy $-E_{0}$, whereas the singleton "Sng" is a lowest-weight module with minimal energy $E_{0}$ (see the section 3 for additional comments on the definition of anti-singletons). From the clear relation between Sng and $\overline{\mathrm{Sng}}$, we can relate the character of the antisingletons to that of the singletons as

$$
\chi_{\overline{\mathrm{Sng}}}^{s o(2,3)}(\beta, \alpha)=\chi_{\mathrm{Sng}}^{s o(2,3)}(-\beta,-\alpha)=\chi_{\mathrm{Sng}}^{\text {so(2,3) }}(\beta, \alpha) .
$$

The last equality holds because the singleton characters are even functions of $\beta$ and $\alpha$ (see $(2.5)$ ). If all the above discussions were free from subtleties, we should be able to 
reproduce the character of the adjoint module (2.26) as the product of the singleton and anti-singleton characters. However, the identity (2.38) already shows that it cannot be so, because the adjoint and twisted adjoint modules have different characters (since they are not isomorphic). More explicitly, we find the following discrepancies

$$
\begin{aligned}
\chi_{\mathrm{Adj}}^{s o(2,3)}(\beta, \alpha) & =\frac{\cosh ^{2} \frac{\beta}{2}+\cos ^{2} \frac{\alpha}{2}}{(\cosh \beta-\cos \alpha)^{2}} \\
\neq \chi_{\mathrm{Rac}}^{s o(2,3)}(\beta, \alpha) \chi_{\mathrm{Rac}}^{s o(2,3)}(\beta, \alpha) & =\frac{\cosh ^{2} \frac{\beta}{2}}{(\cosh \beta-\cos \alpha)^{2}} \\
\neq \chi_{\mathrm{Di}}^{s o(2,3)}(\beta, \alpha) \chi_{\overline{\mathrm{Di}}}^{s o(2,3)}(\beta, \alpha) & =\frac{\cos ^{2} \frac{\alpha}{2}}{(\cosh \beta-\cos \alpha)^{2}} .
\end{aligned}
$$

What went wrong? There are several potential sources of discrepancies. First, it might be due to the problem of change of basis between $X^{m, n ; p, q}$ and $T^{m, n ; p, q}$. Second, it might be a problem of characters: the lowest-weight modules and the highest-weight modules have different radius of convergence for $q$ or, equivalently, for $\beta$. The former one converges for $\beta>0$ while the latter one does so for $\beta<0$. Once the infinite series are evaluated in the convergent region of $\beta$, this region can be analytically continued to the outer region. However, there might be subtleties in handling the characters of lowest-weight modules and highest-weight modules simultaneously. To give away the bottom line already, various considerations (that are presented below) indicate that the relation (2.37) itself, namely the naive twisted Flato-Fronsdal theorem, should be modified.

In order to understand better this discrepancy, let us redo the character computations using the oscillator realization. The higher-spin algebra is spanned by the elements $X^{m, n ; p, q}$ with $m+n+p+q \in 2 \mathbb{N}$, as defined in (2.28), which also form a basis. In the present case, the $s o(2,3)$ subalgebra acts on the elements of the higher-spin algebra through the adjoint action. The generators $X^{m, n ; p, q}$ are also eigenvectors of $E$ and $J_{3}$ :

$$
\left[E, X^{m, n ; p, q}\right]=\frac{m+n-p-q}{2} X^{m, n ; p, q}, \quad\left[J_{3}, X^{m, n ; p, q}\right]=\frac{m-n-p+q}{2} X^{m, n ; p, q} .
$$

This implies that the character associated to the adjoint module is:

$$
\chi_{\text {Adj }}^{\text {so(2,3) }}(\beta, \alpha)=\operatorname{Tr}_{\text {Adj }}\left(e^{-\beta E+i \alpha J_{3}}\right)=\sum_{m+n+p+q \in 2 \mathbb{N}} e^{-\beta \frac{m+n-p-q}{2}+i \alpha \frac{m-n-p+q}{2}} .
$$

Note that the above series is not well-defined because of the infinite degeneracy for a given eigenvalue $E$ and $J_{3}$. However, we can still make some formal manipulations on it. Let us start by separating (2.41) into two parts:

$$
\chi_{\operatorname{Adj}}^{s o(2,3)}(\beta, \alpha)=\sum_{\substack{m+n \in 2 \mathbb{N} \\ p+q \in 2 \mathbb{N}}} e^{-\beta \frac{m+n-p-q}{2}+i \alpha \frac{m-n-p+q}{2}}+\sum_{\substack{m+n \in 2 \mathbb{N}+1 \\ p+q \in 2 \mathbb{N}+1}} e^{-\beta \frac{m+n-p-q}{2}+i \alpha \frac{m-n-p+q}{2}} .
$$

The first series factors as

$$
\begin{aligned}
& \sum_{m+n \in 2 \mathbb{N}} e^{-\frac{\beta}{2}(m+n+1)+i \frac{\alpha}{2}(m-n)} \sum_{p+q \in 2 \mathbb{N}} e^{+\frac{\beta}{2}(p+q+1)-i \frac{\alpha}{2}(p-q)} \\
& \quad=\chi_{\operatorname{Rac}}^{s o(2,3)}(\beta, \alpha) \times \chi_{\operatorname{Rac}}^{s o(2,3)}(-\beta,-\alpha)=\chi_{\operatorname{Rac}}^{s o(2,3)}(\beta, \alpha) \times \chi_{\overline{\operatorname{Rac}} s o(2,3)}^{s}(\beta, \alpha),
\end{aligned}
$$


whereas the second series factors as

$$
\begin{aligned}
& \sum_{m+n \in 2 \mathbb{N}+1} e^{-\frac{\beta}{2}(m+n+1)+i \frac{\alpha}{2}(m-n)} \sum_{p+q \in 2 \mathbb{N}+1} e^{+\frac{\beta}{2}(p+q+1)-i \frac{\alpha}{2}(p-q)} \\
& =\chi_{\mathrm{Di}}^{s o(2,3)}(\beta, \alpha) \times \chi_{\mathrm{Di}}^{s o(2,3)}(-\beta,-\alpha)=\chi_{\mathrm{Di}}^{s o(2,3)}(\beta, \alpha) \times \chi_{\overline{\mathrm{Di}}}^{s o(2,3)}(\beta, \alpha) .
\end{aligned}
$$

Therefore, we find

$$
\chi_{\mathrm{Adj}}^{s o(2,3)}=\chi_{\mathrm{Rac}}^{s o(2,3)} \times \chi_{\overline{\mathrm{Rac}}}^{s o(2,3)}+\chi_{\mathrm{Di}}^{s o(2,3)} \times \chi_{\overline{\mathrm{Di}}}^{s o(2,3)},
$$

which is in accordance with (2.26). Note again that the above manipulation is formal and can be understood only as a regularization procedure. The generators $T^{m, n ; p, q}$ are also eigenvectors, of identical eigenvalues, than the generators $X^{m, n ; p, q}$. However, the basis elements $T^{m, n ; p, q}$ of the space $\operatorname{Rac} \otimes \overline{\operatorname{Rac}}$ are such that $m+n \in 2 \mathbb{N}$ and $p+q \in 2 \mathbb{N}$, therefore the corresponding character is equal to the first sum (2.43) and one finds

$$
\chi_{\mathrm{Rac} \otimes \overline{\operatorname{Rac}}}^{s o(2,3)}=\chi_{\operatorname{Rac}}^{s o(2,3)} \times \chi_{\overline{\operatorname{Rac}}}^{s o(2,3)} .
$$

Similarly, the basis elements $T^{m, n ; p, q}$ of the space $\mathrm{Di} \otimes \overline{\mathrm{Di}}$ are such that $m+n \in 2 \mathbb{N}+1$ and $p+q \in 2 \mathbb{N}+1$, leading to

$$
\chi_{\mathrm{Di} \otimes \overline{\mathrm{Di}}}^{s o(2,3)}=\chi_{\mathrm{Di}}^{s o(2,3)} \times \chi_{\overline{\mathrm{Di}}}^{s o(2,3)} .
$$

These computations suggest a neat conclusion in four dimensions: the heuristic equality (2.37) should be replaced with

$$
\operatorname{Adj}=(\operatorname{Rac} \otimes \overline{\operatorname{Rac}}) \oplus(\operatorname{Di} \otimes \overline{\mathrm{Di}}),
$$

as suggested from the change of basis (2.36) if one properly takes into account the range of the indices.

Let us summarize what we have observed. First, we have seen that the adjoint module of the higher-spin algebra is actually larger than that of the Rac and anti-Rac tensorproduct module. This was manifest in the oscillator analysis and the complementary vector space was identified with the $\mathrm{Di} \otimes \overline{\mathrm{Di}}$ module. In fact, as we shall see in below, the tensor-product module $\operatorname{Rac} \otimes \overline{\operatorname{Rac}}$ fails to cover the entire adjoint module also in higher dimensions. However, the complementary space cannot be interpreted as $\mathrm{Di} \otimes \overline{\mathrm{Di}}$ except in four dimensions. This should be related to the fact that only in four dimensions Rac and Di have the same endomorphism algebra. In other words, the type-A and type-B higher-spin algebras coincide with each other only in four dimensions.

We can also regard the complementary space as a "permuted" Rac module, in the sense that

$$
\chi_{\mathrm{Di}}^{\text {so(2,3) }}\left(i \alpha_{1}, \alpha_{2}\right)=-\chi_{\operatorname{Rac}}^{s o(2,3)}\left(i \alpha_{2}, \alpha_{1}\right) .
$$

Then, the result (2.45) can be viewed as the symmetrization,

$$
\begin{aligned}
\chi_{\mathrm{Adj}}^{s o(2,3)}\left(i \alpha_{1}, \alpha_{2}\right) & =\chi_{\mathrm{Rac}}^{s o(2,3)}\left(i \alpha_{1}, \alpha_{2}\right) \chi_{\overline{\operatorname{Rac}}}^{s o(2,3)}\left(i \alpha_{1}, \alpha_{2}\right)+(1 \leftrightarrow 2) \\
& =\chi_{\mathrm{Di}}^{s o(2,3)}\left(i \alpha_{1}, \alpha_{2}\right) \chi_{\overline{\mathrm{Di}}}^{s o(2,3)}\left(i \alpha_{1}, \alpha_{2}\right)+(1 \leftrightarrow 2) .
\end{aligned}
$$


In fact, the character of the adjoint module is clearly symmetric under the exchange of $i \beta$ and $\alpha$ as it is the sum of the characters of $\mathcal{D}(1-s, s-1)$ given in (2.22) having this property. On the other hand, the product of the singleton and anti-singleton characters is generically asymmetric as we can see in (2.39). Hence, the simplest way to relate this asymmetric function to the symmetric one would be by the symmetrization of (2.50). The relation (2.50) at the level of the characters can be translated back to the modules as

$$
\begin{aligned}
\operatorname{Adj} & =(\operatorname{Rac} \otimes \overline{\operatorname{Rac}}) \oplus(\tau(\operatorname{Rac}) \otimes \tau(\overline{\operatorname{Rac}})) \\
& =(\operatorname{Di} \otimes \overline{\mathrm{Di}}) \oplus(\tau(\mathrm{Di}) \otimes \tau(\overline{\mathrm{Di}})),
\end{aligned}
$$

where $\tau$ is the weight-space map exchanging the two Cartan generators, and hence can be viewed as an element of the Weyl group of $s o(2,3)$ (quotiented by the normalizer subgroup of $\operatorname{Rac} \otimes \overline{\operatorname{Rac}}$ or $\mathrm{Di} \otimes \overline{\mathrm{Di}})$. In order to obtain the second equality in (2.51), we used the relations

$$
\begin{aligned}
\tau(\mathrm{Rac}) \otimes \tau(\overline{\mathrm{Rac}}) & =\mathrm{Di} \otimes \overline{\mathrm{Di}}, \\
\tau(\mathrm{Di}) \otimes \tau(\overline{\mathrm{Di}}) & =\operatorname{Rac} \otimes \overline{\mathrm{Rac}},
\end{aligned}
$$

which can also be used in order to relate (2.48) and (2.51). Since the "symmetrized" tensor product in (2.51) can be generalized to higher dimensions, we propose it as a refined version for the twisted Flato-Fronsdal theorem. ${ }^{5}$ Interestingly, the idea of symmetrization works in higher dimensions as well as for the higher order singletons, as we shall show in the following sections.

Before moving to general dimensions, let us comment on the unitarity of modules. The mere tensor product of two unitary modules (Sng and $\overline{\text { Sng }}$ ) should not result in a nonunitary module (the adjoint module) in general. Hence, at first glance, this indicates that a refinement is needed in the naive twisted Flato-Fronsdal theorem (2.37). But, the issue is in fact more subtle: the new additional term in the refined twisted Flato-Fronsdal theorem can be written either as $\mathrm{Di} \otimes \overline{\mathrm{Di}}$ in $(2.48)$ or as $\tau(\mathrm{Rac}) \otimes \tau(\overline{\mathrm{Rac}})$ in $(2.51)$. Since Di is unitary while $\tau(\mathrm{Rac})$ is not, the (non-)unitarity of the refinement term is not clear. This subtlety can be related to the possibility that the relation (2.48) or (2.51) may require a suitable completion of the corresponding vector spaces. Indeed, the change of basis (2.36) relating the two modules expresses the generator $T^{m, n ; p, q}$ as an infinite linear combination of the generators $X^{m, n ; p, q}$, and the norm of the former may diverge even though each summand has a finite norm. This subtle point will be left somewhat implicit in expressions like (2.48) and (2.51). This issue may be related to the regularization of the adjoint module character provided by the twisted Flato-Fronsdal theorem.

\section{General dimensions}

In this section, we shall provide more evidences of the "symmetrization" prescription for the relation between singleton and adjoint module character, by examining the type-A and type-B models in any dimension.

\footnotetext{
${ }^{5}$ Let us stress that the twisted Flato-Fronsdal theorem (2.51) essentially relies on the change of basis in the higher-spin algebra (more precisely, a suitable completion thereof). In other words, our proof does not actually relies on characters.
} 
For a smooth demonstration, let us provide here some details about the $s o(d)$ and $s o(2, d)$ characters. A unitary irreducible representation of $s o(d)$ is entirely determined by a highest weight $\ell=\left(\ell_{1}, \ldots, \ell_{r}\right)$ with $r=[d / 2]$ the integer part of $d / 2$ which is also the rank of $s o(d)$, and $\ell_{1} \geqslant \cdots \geqslant \ell_{r-1} \geqslant\left|\ell_{r}\right|$ (the last number $\ell_{r}$ can be negative only for $s o(2 r))$ are either all integers or all half-integers. Its character is given by

$$
\chi_{\ell}^{s o(d)}(\boldsymbol{x})=\operatorname{Tr}_{\ell}\left[x_{1}^{M^{12}} \cdots x_{r}^{M^{2 r-12 r}}\right]=\left\{\begin{array}{cc}
\frac{\operatorname{det}\left[x_{i}{ }^{k}{ }-x_{i}{ }^{-k_{j}}\right]}{\Delta^{(r)}(\boldsymbol{x}) \prod_{i=1}^{r}\left(x_{i}{ }^{\frac{1}{2}}-x_{i}{ }^{-\frac{1}{2}}\right)} & {[d=2 r+1]} \\
\frac{\operatorname{det}\left[x_{i}{ }^{k}{ }_{j}+x_{i}{ }^{-k_{j}}\right]+\operatorname{det}\left[x_{i}{ }^{k_{j}}-x_{i}{ }^{-k_{j}}\right]}{2 \Delta^{(r)}(\boldsymbol{x})} & {[d=2 r]}
\end{array},\right.
$$

with $\boldsymbol{x}=\left(x_{1}, \ldots, x_{r}\right)$ and $k_{i}=\ell_{i}+\frac{d}{2}-i$. Here $\Delta^{(r)}(\boldsymbol{x})$ is the Vandermonde determinant,

$$
\Delta^{(r)}(\boldsymbol{x})=\prod_{1 \leqslant i<j \leqslant r}\left(x_{i}+x_{i}^{-1}-x_{j}-x_{j}^{-1}\right) .
$$

Notice that the character formulae displayed in the previous section can be recovered after setting $x_{k}=e^{i \alpha_{k}}$ with $k=1, \ldots, r$ (and setting $r=1$ since $d=3$ there).

Turning now to the non-compact Lie algebra $s o(2, d)$, any of its irreducible lowestweight modules can be described in terms of (quotients of) lowest-weight generalized Verma modules $\mathcal{V}(\Delta, \ell)$ (see appendix A for conventions and technical details). The character of the latter module is given by

$$
\chi_{\mathcal{V}(\Delta, \ell)}^{s o(2, d)}(q, \boldsymbol{x})=\operatorname{Tr}_{\mathcal{V}(\Delta, \ell)}\left[q^{M^{0^{\prime} 0}} x_{1}^{M^{12}} \cdots x_{r}^{M^{2 r-12 r}}\right]=q^{\Delta} \mathcal{P}_{d}(q, \boldsymbol{x}) \chi_{\ell}^{s o(d)}(\boldsymbol{x}),
$$

where the function $\mathcal{P}_{d}(q, \boldsymbol{x})$ defined as

$$
\mathcal{P}_{d}(q, \boldsymbol{x})=\frac{1}{(1-q)^{d-2 r}} \prod_{i=1}^{r} \frac{1}{\left(1-q x_{i}\right)\left(1-q x_{i}^{-1}\right)}=\chi_{\mathcal{V}(0, \mathbf{0})}^{s o(2,)}(q, \boldsymbol{x}),
$$

is the character of the module associated with the trivial weight.

The contragredient representation carried by the dual module of a module $M$ has the opposite quantum numbers ${ }^{6}$ with respect to $M$. For a given lowest-weight module $M$, there exists a highest-weight module $\bar{M}$ with exactly the opposite quantum numbers. We shall refer to this highest-weight module as "anti- $M$ " and equate it with the dual module, disregarding potential subtleties of infinite dimensional vector space. Then, the characters of the anti-module $\bar{M}$ is simply related to that of the module $M$ as

$$
\chi_{\bar{M}}^{s o(2, d)}(q, \boldsymbol{x})=\chi_{M}^{s o(2, d)}\left(q^{-1}, \boldsymbol{x}^{-1}\right)
$$

where $\boldsymbol{x}^{-1}=\left(x_{1}^{-1}, x_{2}^{-1}, \ldots, x_{r}^{-1}\right)$.

\footnotetext{
${ }^{6}$ Recall that, given a representation $(V, \rho)$ of a semisimple Lie algebra $\mathfrak{g}$, the contragredient representation $\left(V^{*}, \rho^{*}\right)$ is defined as $\left(\rho^{*}(x) \cdot \phi\right)(v)=\phi(\rho \circ \tau(x) \cdot v)$ for $x \in \mathfrak{g}, v \in V, \phi \in V^{*}$ and $\tau$ the Chevalley involution. This automorphism of $\mathfrak{g}$ acts on the Cartan subalgebra generators $H_{i}$ and the ladders operators $E_{\alpha}$ as $\tau\left(H_{i}\right)=-H_{i}$ and $\tau\left(E_{\alpha}\right)=-E_{-\alpha}$.
} 
For future use, let us enlist a few properties of the function $\mathcal{P}_{d}$ and of the $s o(d)$ characters. First, $\mathcal{P}_{d}(q, \boldsymbol{x})$ satisfies

$$
\mathcal{P}_{d}\left(q^{-1}, \boldsymbol{x}^{-1}\right)=\mathcal{P}_{d}\left(q^{-1}, \boldsymbol{x}\right)=(-q)^{d} \mathcal{P}_{d}(q, \boldsymbol{x}),
$$

and can be expressed as a series of $s o(d)$ character as [11]

$$
\mathcal{P}_{d}(q, \boldsymbol{x})=\sum_{s, n=0}^{\infty} q^{s+2 n} \chi_{s}^{s o(d)}(\boldsymbol{x})
$$

where $\chi_{s}^{s o(d)}$ denotes the spin- $s$ character of $s o(d)$, corresponding to the highest weight $(s, 0, \ldots, 0)$. Finally, the $s o(2+d)$ character of the irrep $\left(\ell_{0}, \ell\right)$ can be written in terms of the $s o(d)$ character for the irrep $\boldsymbol{\ell}$ as

$$
\chi_{\left(\ell_{0}, \ell\right)}^{s o(2+d)}\left(x_{0}, \boldsymbol{x}\right)=\sum_{k=0}^{r} \mathcal{P}_{d}\left(x_{k}, \boldsymbol{x}_{k}\right) \times\left\{\begin{array}{cc}
\left(x_{k}^{-\ell_{0}}-x_{k}^{\ell_{0}+d}\right) \chi_{\ell}^{s o(d)}\left(\boldsymbol{x}_{k}\right) & {[d=2 r+1]} \\
{\left[x_{k}^{-\ell_{0}} \chi_{\boldsymbol{\ell}_{-}}^{s o(d)}\left(\boldsymbol{x}_{k}\right)+x_{k}^{\ell_{0}+d} \chi_{\boldsymbol{\ell}_{+}}^{s o(d)}\left(\boldsymbol{x}_{k}\right)\right]} & {[d=2 r]}
\end{array}\right.
$$

where $\boldsymbol{x}_{k}=\left(x_{0}, \ldots, x_{k-1}, x_{k+1}, \ldots, x_{r}\right)$ and $\boldsymbol{\ell}_{ \pm}=\left(\ell_{1}, \ldots, \ell_{r-1}, \pm \ell_{r}\right)$. This identity will play a key role in uncovering simple relations between the singleton and adjoint module characters, and can be derived from the Weyl character formula (see appendix B). It is worth noting that the identity (3.8) can be also viewed as a relation between the $s o(2+d)$ character and the $s o(2, d)$ character of $\mathcal{V}(\Delta, \ell)$ :

$$
\chi_{\left(\ell_{0}, \ell\right)}^{s o(2+d)}\left(x_{0}, \boldsymbol{x}\right)=\sum_{k=0}^{r}\left\{\begin{array}{cc}
\chi_{\mathcal{V}\left(-\ell_{0}, \ell\right)}^{s o(2, d)}\left(x_{k}, \boldsymbol{x}_{k}\right)-\chi_{\mathcal{V}\left(\ell_{0}+d, \ell\right)}^{s o(2, d)}\left(x_{k}, \boldsymbol{x}_{k}\right) & {[d=2 r+1]} \\
\chi_{\mathcal{V}\left(-\ell_{0}, \ell_{-}\right)}^{s o(2, d)}\left(x_{k}, \boldsymbol{x}_{k}\right)+\chi_{\mathcal{V}\left(\ell_{0}+d, \ell_{+}\right)}^{s o(2, d)}\left(x_{k}, \boldsymbol{x}_{k}\right) & {[d=2 r]}
\end{array} .\right.
$$

Another property of the $s o(d)$ characters that will prove useful in the subsequent sections is the following:

$$
\chi_{\left(\ell_{1}, \ldots, \ell_{j-1}, \ell_{j}-1, \ell_{j+1}+1, \ell_{j+2}, \ldots, \ell_{r}\right)}^{s o(d)}(\boldsymbol{x})=-\chi_{\left(\ell_{1}, \ldots, \ell_{j-1}, \ell_{j+1}, \ell_{j}, \ell_{j+2}, \ldots, \ell_{r}\right)}^{s o(d)}(\boldsymbol{x}),
$$

as it implies in particular that the character with the label

$$
\left(\ell_{1}, \ldots, \ell_{j-1}, \ell-1, \ell, \ell_{j+2}, \ldots, \ell_{r}\right)
$$

identically vanishes. Equipped with the above identities, let us consider the type-A and type-B higher-spin theories in any dimension, which are based on the scalar and spinor singletons respectively.

\subsection{Type A}

Let us begin with the type-A massless higher-spin gravity in $d+1$ dimensions. It is expected to be dual to the $\mathrm{U}(N)$ free scalar CFT in $d$ dimensions. The free conformal scalar field carries nothing but the spin-0 singleton representation of $s o(2, d)$ :

$$
\mathcal{D}\left(\frac{d-2}{2}, \mathbf{0}\right) \equiv \frac{\mathcal{V}\left(\frac{d-2}{2}, \mathbf{0}\right)}{\mathcal{D}\left(\frac{d+2}{2}, \mathbf{0}\right)},
$$




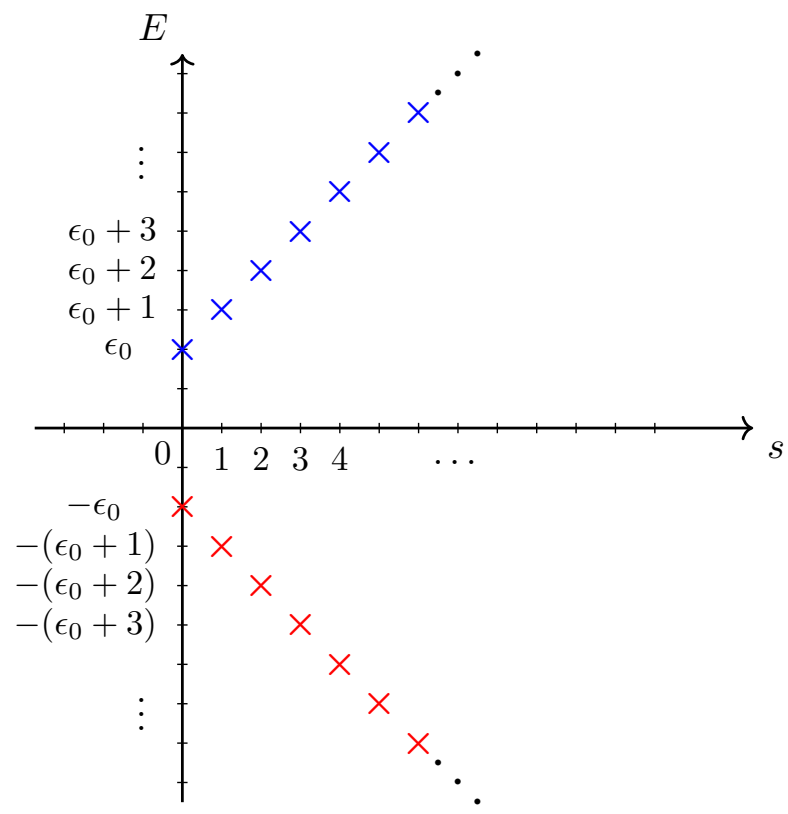

Figure 1. Weight diagram of the scalar singleton, or Rac (blue crosses) and of the scalar antisingleton, or anti-Rac (red crosses).

that, from now on, we shall refer to as "Rac", thereby extending the four-dimensional terminology to any $d$. Note that $\mathbf{0}$ stands for the trivial weight $(0, \ldots, 0)$. Using (3.3), one computes the character of the quotient in (3.12):

$$
\chi_{\operatorname{Rac}}^{s o(2, d)}(q, \boldsymbol{x})=q^{\frac{d-2}{2}}\left(1-q^{2}\right) \mathcal{P}_{d}(q, \boldsymbol{x}) .
$$

The energy eigenvalues of this representation is bounded from below, hence it is a lowestweight representation. One can define an analogous representation whose energy is now bounded from above. We refer to the corresponding module as anti-singleton $\overline{\text { Rac }}$ and its character is simply related to that of the singleton as

$$
\chi_{\overline{\operatorname{Rac}}}^{s o(2, d)}(q, \boldsymbol{x})=\chi_{\operatorname{Rac}}^{s o(2, d)}\left(q^{-1}, \boldsymbol{x}^{-1}\right)=\chi_{\operatorname{Rac}}^{s o(2, d)}\left(q^{-1}, \boldsymbol{x}\right) .
$$

Notice that the character of the Rac and $\overline{R a c}$ modules are simply related to each other, using (3.6), by a sign depending on the parity of $d$, namely,

$$
\chi_{\overline{\operatorname{Rac}}}^{s o(2, d)}(q, \boldsymbol{x})=(-1)^{d+1} \chi_{\operatorname{Rac}}^{s o(2, d)}(q, \boldsymbol{x}) .
$$

Using the property (3.7), the character of Rac and $\overline{\text { Rac }}$ can be also expressed as

$$
\chi_{\operatorname{Rac}}^{s o(2, d)}(q, \boldsymbol{x})=\sum_{s=0}^{\infty} q^{\epsilon_{0}+s} \chi_{s}^{s o(d)}(\boldsymbol{x}), \quad \chi_{\overline{\operatorname{Rac}}}^{s o(2, d)}(q, \boldsymbol{x})=\sum_{s=0}^{\infty} q^{-\epsilon_{0}-s} \chi_{s}^{s o(d)}(\boldsymbol{x}),
$$

where $\epsilon_{0}:=(d-2) / 2$. From the above formulae, the weight space of the Rac and $\overline{\operatorname{Rac}}$ representations respectively can be read off. Their weights are depicted in figure 1. 
Now, let us see how the character of the adjoint module can be related to the Rac module. The higher-spin algebra of type-A theory is the collection of the $s o(2+d)$ irrep $(s-1, s-1):=(s-1, s-1,0, \ldots, 0)$ for $s=1,2, \ldots, \infty$. Applying the identity (3.8) to these irreps, we obtain

$$
\chi_{(s-1, s-1)}^{s o(2+d)}\left(x_{0}, \boldsymbol{x}\right)=\sum_{k=0}^{r} \mathcal{P}_{d}\left(x_{k}, \boldsymbol{x}_{k}\right)\left(x_{k}^{1-s}+(-1)^{d} x_{k}^{s+d-1}\right) \chi_{s-1}^{s o(d)}\left(\boldsymbol{x}_{k}\right) .
$$

In the right-hand-side of the equation, the last two factors can be summed over $s$ by using the properties (3.6) and (3.7) as

$$
\sum_{s=1}^{\infty}\left(x_{k}^{1-s}+(-1)^{d} x_{k}^{s+d-1}\right) \chi_{s-1}^{s o(d)}\left(\boldsymbol{x}_{k}\right)=\left(x_{k}^{-1}-x_{k}\right)\left(x_{k}-x_{k}^{-1}\right) \mathcal{P}_{d}\left(x_{k}^{-1}, \boldsymbol{x}_{k}\right) .
$$

Notice that when summing the above expression, we used the identity (3.7) as if it was valid simultaneously in both domains of convergence $\left|x_{k}\right|<1$ and $\left|x_{k}\right|>1$ for all $k=0,1, \cdots, r$. Multiplying the above equation by $\mathcal{P}_{d}\left(x_{k}, \boldsymbol{x}_{k}\right)$ and symmetrizing over $k$, we finally obtain

$$
\chi_{\operatorname{Adj}}^{s o(2+d)}\left(x_{0}, \boldsymbol{x}\right)=\sum_{s=1}^{\infty} \chi_{(s-1, s-1)}^{s o(2+d)}\left(x_{0}, \boldsymbol{x}\right)=\sum_{k=0}^{r} \chi_{\operatorname{Rac}}^{s o(2, d)}\left(x_{k}, \boldsymbol{x}_{k}\right) \chi_{\overline{\operatorname{Rac}}}^{s o(2, d)}\left(x_{k}, \boldsymbol{x}_{k}\right),
$$

where one should remember that $\boldsymbol{x}_{k}=\left(x_{0}, \ldots, x_{k-1}, x_{k+1}, \ldots, x_{r}\right)$. Hence, the sum over the characters corresponding to all the $s o(2+d)$ two-row rectangular Young diagrams (i.e. the diagrams $(2.20)$ for $s=1,2, \ldots, \infty)$ is equal to the product of the characters of a Rac and an anti-Rac symmetrized over all variables.

\section{$3.2 \quad$ Type B}

The type-B massless higher-spin gravity in $d+1$ dimensions is conjectured to be dual to the free CFT with Dirac spinor in $d$ dimensions.

Even $\boldsymbol{d}+\mathbf{1}$ dimensions. For $d+1$ even (that is $d$ odd), this free Dirac spinor carries the spin- $\frac{1}{2}$ singleton representation,

$$
\text { Di }:=\mathcal{D}\left(\frac{d-1}{2}, \frac{\mathbf{1}}{\mathbf{2}}\right)=\frac{\mathcal{V}\left(\frac{d-1}{2}, \frac{\mathbf{1}}{\mathbf{2}}\right)}{\mathcal{V}\left(\frac{d+1}{2}, \frac{\mathbf{1}}{2}\right)}
$$

with $\frac{1}{2}=\left(\frac{1}{2}, \ldots, \frac{1}{2}\right)$. The character of Di has the form [11],

$$
\chi_{\mathrm{Di}}^{s o(2, d)}(q, \boldsymbol{x})=q^{\frac{d-1}{2}}(1-q) \mathcal{P}_{d}(q, \boldsymbol{x}) \chi_{\frac{1}{2}}^{s o(d)}(\boldsymbol{x}),
$$

and the character of anti-Di is simply related to the above as

$$
\chi_{\overline{\mathrm{Di}}}^{s o(2, d)}(q, \boldsymbol{x})=\chi_{\mathrm{Di}}^{s o(2, d)}\left(q^{-1}, \boldsymbol{x}^{-1}\right)=\chi_{\mathrm{Di}}^{s o(2, d)}\left(q^{-1}, \boldsymbol{x}\right),
$$

since $\chi_{\frac{1}{2}}^{s o(d)}\left(\boldsymbol{x}^{-1}\right)=\chi_{\frac{1}{2}}^{s o(d)}(\boldsymbol{x})$. From the identity (3.7), we derive another useful identity,

$$
(1-q) \mathcal{P}_{d}(q, \boldsymbol{x}) \chi_{\frac{1}{2}}^{s o(d)}(\boldsymbol{x})=\sum_{s=0}^{\infty} q^{s} \chi_{\left(s+\frac{1}{2}, \frac{1}{2}^{r-1}\right)}^{s o(d)}(\boldsymbol{x}),
$$


where the notation $c^{m}$ in a weight stands for a sequence of $m$ identical entries $c$ : for instance, $\left(s+\frac{1}{2}, \frac{1}{2}^{r-1}\right)=\left(s+\frac{1}{2}, \frac{1}{2}, \ldots, \frac{1}{2}\right)$. The identity (3.23) implies that, similarly to the Rac, the character of Di can be written as

$$
\left.\chi_{\mathrm{Di}}^{s o(2, d)}(q, \boldsymbol{x})=\sum_{s=0}^{\infty} q^{\frac{d-1}{2}+s} \chi_{\left(s+\frac{1}{2}, \frac{1}{2}\right.}^{s-1}\right)(\boldsymbol{x}) .
$$

Notice that the character of the Di singleton is actually identical to that of its anti-singleton $\overline{\mathrm{Di}}$ for $d=2 r+1$ :

$$
\chi_{\overline{\mathrm{Di}}}^{s o(2,2 r+1)}(q, \boldsymbol{x})=\chi_{\mathrm{Di}}^{s o(2,2 r+1)}(q, \boldsymbol{x}),
$$

similarly to the Rac case.

We want to relate this character to that of the adjoint module of type-B higher-spin algebra. In the section 3.1, we started from the adjoint module and showed that its character can be written in terms of the Rac and anti-Rac characters. In the type-B case, its higher-spin algebra was identified in [8], so we can proceed, in principle, in the same way. However, the utility of the twisted Flato-Fronsdal theorems is actually to identify the higher-spin algebra directly from the underlying singleton modules. Hence, for type-B theory let us proceed in the opposite way to the type-A case: we begin with the Di and anti-Di character and find the character of the adjoint module.

The starting point is the product of the Di and anti-Di characters,

$$
\chi_{\mathrm{Di}}^{s o(2, d)}(q, \boldsymbol{x}) \chi_{\overline{\mathrm{Di}}}^{s o(2, d)}(q, \boldsymbol{x})=\left(1-q+1-q^{-1}\right) \mathcal{P}_{d}(q, \boldsymbol{x}) \mathcal{P}_{d}\left(q^{-1}, \boldsymbol{x}\right) \chi_{\frac{1}{2}}^{s o(d)}(\boldsymbol{x}) \chi_{\frac{1}{2}}^{s o(d)}(\boldsymbol{x}) .
$$

Using (3.6) and (3.23), the above can be expressed as

$$
\begin{aligned}
& \chi_{\mathrm{Di}}^{s o(2, d)}(q, \boldsymbol{x}) \chi_{\frac{\mathrm{Di}}{s o(2, d)}(q, \boldsymbol{x})}^{\infty} \\
& \quad=\mathcal{P}_{d}(q, \boldsymbol{x}) \sum_{s=0}^{\infty}\left(q^{-s}-q^{s+d}\right) \chi_{\left(s+\frac{1}{2}, \frac{1^{2}}{2}\right.}^{s o(d)}(\boldsymbol{x}) \chi_{\frac{1}{2}}^{s o(d)}(\boldsymbol{x}) \\
& \quad=\mathcal{P}_{d}(q, \boldsymbol{x}) \sum_{s=0}^{\infty} \sum_{m=0}^{r-1}\left(q^{-s}-q^{s+d}\right)\left[\chi_{\left(s, 1^{m}\right)}^{s o(d)}(\boldsymbol{x})+\chi_{\left(s+1,1^{m}\right)}^{s o(d)}(\boldsymbol{x})\right] .
\end{aligned}
$$

In the last line, the product of two $s o(d)$ characters is decomposed in terms of other $s o(d)$ characters using the decomposition rule (see e.g. [51] for the general decomposition rule of the tensor product of two $s o(d)$-modules, recalled in [52]):

$$
\left(s+\frac{1}{2}, \frac{1}{2}^{r-1}\right) \otimes\left(\frac{1}{2}^{r}\right)=\bigoplus_{m=0}^{r-1}\left[\left(s, 1^{m}\right) \oplus\left(s+1,1^{m}\right)\right] .
$$

Note that the modules with improper weight labels do not contribute, namely the first term in the summand on the right-hand-side of the above decomposition is absent, when $m \neq 0$, for $s=0$. This can be also viewed from the fact that the characters associated with the improper labels that appear in (3.28) identically vanish, due to the identity (3.10). 
Finally, symmetrizing over the variables, we get

$$
\sum_{k=0}^{r} \chi_{\mathrm{Di}}^{s o(2, d)}\left(x_{k}, \boldsymbol{x}_{k}\right) \chi_{\overline{\mathrm{Di}}}^{s o(2, d)}\left(x_{k}, \boldsymbol{x}_{k}\right)=\sum_{s=0}^{\infty} \sum_{m=0}^{r-1}\left[\chi_{\left(s, s, 1^{m}\right)}^{s o(2+d)}\left(x_{0}, \boldsymbol{x}\right)+\chi_{\left(s, s+1,1^{m}\right)}^{s o(2+d)}\left(x_{0}, \boldsymbol{x}\right)\right] .
$$

Once again, the characters with improper labels identically vanish (i.e. the second term on the right-hand-side), hence we derive in the end,

$$
\sum_{k=0}^{r} \chi_{\mathrm{Di}}^{s o(2, d)}\left(x_{k}, \boldsymbol{x}_{k}\right) \times \chi_{\overline{\mathrm{Di}}}^{s o(2, d)}\left(x_{k}, \boldsymbol{x}_{k}\right)=\chi_{0}^{s o(2+d)}\left(x_{0}, \boldsymbol{x}\right)+\sum_{s=2}^{\infty} \sum_{m=0}^{r} \chi_{\left(s-1, s-1,1^{m}\right)}^{s o(2+d)}\left(x_{0}, \boldsymbol{x}\right),
$$

where $\chi_{0}^{s o(2+d)}=1$ corresponds to the identity of the higher-spin algebra. The content of the right-hand-side precisely coincides with the adjoint module of the type-B higher-spin algebra identified in [8].

Odd $\boldsymbol{d}+\mathbf{1}$ dimensions. For even values $d=2 r$ of the boundary dimension, one can consider a chiral (or anti-chiral) spinor singleton, i.e. whose spin is either $\frac{1}{2}+$ or $\frac{1}{2}$. The corresponding $s o(2, d)$ module is [48]

$$
\mathrm{Di}_{ \pm}:=\mathcal{D}\left(\frac{d-1}{2}, \frac{\mathbf{1}}{\mathbf{2}}_{ \pm}\right)=\frac{\mathcal{V}\left(\frac{d-1}{2}, \frac{1}{2}_{ \pm}\right)}{\mathcal{D}\left(\frac{d+1}{2}, \frac{1}{2}_{\mp}\right)}
$$

and its character reads

$$
\chi_{\mathrm{Di}_{ \pm}}^{s o(2,2 r)}(q, \boldsymbol{x})=q^{\frac{2 r-1}{2}}\left(\chi_{\frac{1}{\mathbf{2}_{ \pm}}}^{s o(2 r)}(\boldsymbol{x})-q \chi_{\frac{1}{\mathbf{1}_{\mp}}}^{s o(2 r)}(\boldsymbol{x})\right) \mathcal{P}_{2 r}(q, \boldsymbol{x}) .
$$

Using the expression (3.7) of $\mathcal{P}_{d}$, we can rewrite the character as

$$
\chi_{\mathrm{Di}_{ \pm}}^{s o(2,2 r)}(q, \boldsymbol{x})=\sum_{s=0}^{\infty} q^{\frac{2 r-1}{2}+s} \chi_{\left(s+\frac{1}{2}, \frac{1}{2}_{ \pm}^{r-1}\right)}^{s o(2 r)}(\boldsymbol{x}) .
$$

The characters of the anti-Di modules are given, by definition, as

$$
\chi_{\overline{\mathrm{Di}}_{ \pm}}^{s o(2,2 r)}(q, \boldsymbol{x})=\chi_{\mathrm{Di}_{ \pm}}^{s o(2,2 r)}\left(q^{-1}, \boldsymbol{x}^{-1}\right) .
$$

But now differently from the previous cases, the symmetry property of the $s o(2 r)$ character $\chi_{\left(s+\frac{1}{2}, \frac{1}{2}{ }_{ \pm}^{r-1}\right)}^{s o(2 r)}(\boldsymbol{x})$ depends on the parity of $r$ :

$$
\chi_{\left(s+\frac{1}{2}, \frac{1}{2}_{ \pm}^{r-1}\right)}^{s o(2 r)}\left(\boldsymbol{x}^{-1}\right)=\left\{\begin{array}{ll}
\chi_{\left(s+\frac{1}{2}, \frac{1}{2}_{ \pm}^{r-1}\right)}^{s o(2 r)}(\boldsymbol{x}) & {[\text { even } r]} \\
\chi_{\left(s+\frac{1}{2}, \frac{1}{2}_{\mp}^{r-1}\right)}^{s o(2 r)}(\boldsymbol{x}) & {[\text { odd } r]}
\end{array} .\right.
$$

As a consequence, the relation between the characters of the anti-Di and the Di modules also depend on the parity of $r$ :

$$
\chi_{\overline{\mathrm{Di}}_{ \pm}}^{s o(2,2 r)}(q, \boldsymbol{x})=-\left\{\begin{array}{ll}
\chi_{\mathrm{Di}_{\mp}}^{s o(2,2 r)}(q, \boldsymbol{x}) & {[\text { even } r]} \\
\chi_{\mathrm{Di}_{ \pm}}^{s o(2,2 r)}(q, \boldsymbol{x}) & {[\text { odd } r]}
\end{array} .\right.
$$


Due to the possible chirality of the Di singleton, only a subset of the mixed-symmetry fields, present in the even-dimensional twisted Flato-Fronsdal theorem (3.30), will appear. Let us start by recalling the generalized Flato-Fronsdal theorem (first derived in [8]), before deriving the corresponding twisted version. To do so, we will need the following decomposition rules of $s o(2 r)$,

$$
\frac{\mathbf{1}}{\mathbf{2}}_{ \pm} \otimes \frac{\mathbf{1}}{\mathbf{2}}=\bigoplus_{m=0}^{\left[\frac{r}{2}\right]} 1_{ \pm}^{r-2 m}, \quad \text { and } \quad \frac{\mathbf{1}}{\mathbf{2}} \otimes \frac{\mathbf{1}}{\mathbf{2}}=\bigoplus_{m=0}^{\left[\frac{r-1}{2}\right]} 1^{r-1-2 m}
$$

together with

$$
\left(s+\frac{1}{2}, \frac{1}{2}_{ \pm}^{r-1}\right) \otimes \frac{\mathbf{1}}{\mathbf{2}} \pm=\bigoplus_{m=0}^{\left[\frac{r-1}{2}\right]}\left(s+1,1_{ \pm}^{r-1-2 m}\right) \oplus \bigoplus_{m=0}^{\left[\frac{r-2}{2}\right]}\left(s, 1^{r-2-2 m}\right)
$$

and

$$
\left(s+\frac{1}{2}, \frac{1}{2}_{ \pm}^{r-1}\right) \otimes \frac{\mathbf{1}}{\mathbf{2}} \mp=\bigoplus_{\mp=0}^{\left[\frac{r-2}{2}\right]}\left(s+1,1^{r-2-2 m}\right) \oplus \bigoplus_{m=0}^{\left[\frac{r-1}{2}\right]}\left(s, 1_{ \pm}^{r-1-2 m}\right) .
$$

In the following, we will treat separately the case of odd and even rank $r$ :

- Even rank $r=2 k$. In this case, the tensor product of two singletons of the same chirality decomposes into a direct sum of hook-shaped massless fields whose first columns are of all even heights from 0 to $r$, together with a collection of massive $p$-forms with $p$ taking all even values from 0 to $r$. Explicitly,

$$
\left(\chi_{\mathrm{Di}_{ \pm}}^{s o(2, d)}\right)^{2}=\sum_{m=0}^{k} \chi_{\mathcal{D}\left(d-1,1_{ \pm}^{2 m}\right)}^{s o(2, d)}+\sum_{s=2}^{\infty} \sum_{m=0}^{k-1} \chi_{\mathcal{D}\left(s+d-2, s, 1_{ \pm}^{2 m+1}\right)}^{s o(2, d)} .
$$

In particular, this decomposition contains the massive scalar $\mathcal{D}(d-1, \mathbf{0})$ as well as massless fields whose first columns are of maximal height $r$ and of the same chirality as the Di singletons. The totally symmetric fields are however absent from this spectrum, they are instead contained in the tensor product of two singletons of opposite chiralities, together with hook-shaped massless fields and massive $p$-forms whose first column is of odd height:

$$
\chi_{\mathrm{Di}_{+}}^{s o(2, d)} \times \chi_{\mathrm{Di}_{-}}^{s o(2, d)}=\sum_{s=1}^{\infty} \chi_{\mathcal{D}(s+d-2, s)}^{s o(2, d)}+\sum_{s=2}^{\infty} \sum_{m=1}^{k-1} \chi_{\mathcal{D}\left(s+d-2, s, 1^{2 m}\right)}^{s o(2, d)}+\sum_{m=1}^{k-1} \chi_{\mathcal{D}\left(d-1,1^{2 m+1}\right)}^{s o(2, d)} .
$$

Using the $s o(d)$ tensor product rules recalled previously, as well as the decomposition (3.33), one can show that the tensor product of a spinor singleton of fixed chirality with its anti-singleton decomposes as

$$
\sum_{j=0}^{r} \chi_{\mathrm{Di}_{ \pm}}^{s o(2, d)}\left(x_{j}, \boldsymbol{x}_{j}\right) \times \chi_{\overline{\mathrm{Di}}_{ \pm}}^{s o(2, d)}\left(x_{j}, \boldsymbol{x}_{j}\right)=\chi_{0}^{s o(2+d)}\left(x_{0}, \boldsymbol{x}\right)+\sum_{s=2}^{\infty} \sum_{m=0}^{k-1} \chi_{\left(s-1, s-1,1^{2 m}\right)}^{s o(2+d)}\left(x_{0}, \boldsymbol{x}\right)
$$


whereas the tensor product of the $\mathrm{Di}_{+}$singleton with the $\overline{\mathrm{Di}}$ - anti-singleton yields

$$
\sum_{j=0}^{r} \chi_{\mathrm{Di}_{ \pm}}^{s o(2, d)}\left(x_{j}, \boldsymbol{x}_{j}\right) \times \chi_{\overline{\mathrm{Di}}_{\mp}}^{s o(2, d)}\left(x_{j}, \boldsymbol{x}_{j}\right)=\sum_{s=2}^{\infty} \sum_{m=0}^{k-1} \chi_{\left(s-1, s-1,1_{ \pm}^{2 m+1}\right)}^{s o(2+d)}\left(x_{0}, \boldsymbol{x}\right) .
$$

The modules appearing in the same/opposite-chirality twisted Flato-Fronsdal theorem $(3.42) /(3.43)$ correspond to the Killing tensors associated to the massless fields appearing in the opposite/same-chirality Flato-Fronsdal theorem $(3.41) /(3.40)$. This crossed correspondence may look problematic if we consider the (anti-)chiral projection, but it is in fact consistent since, in the non-minimal type-B theory, we have to take the tensor product of $\mathrm{Di}_{ \pm}$and its complex conjugate for the bulk spectrum. In $d=4 k$ dimensions, the complex conjugate flips the chirality, hence the bulk spectrum is (3.41), which is compatible with $(3.42)[8,26,53]$.

- Odd rank $r=2 k+1$. In this case, the tensor product of two singletons of the same chirality decomposes into a direct sum of hook-shaped massless fields whose first columns are of any odd height, together with a collection of massive $p$-forms with $p$ taking all odd values from 1 to $r$. Explicitly,

$$
\left(\chi_{\mathrm{Di}_{ \pm}}^{s o(2, d)}\right)^{2}=\sum_{s=1}^{\infty} \chi_{\mathcal{D}(s+d-2, s)}^{s o(2, d)}+\sum_{s=2}^{\infty} \sum_{m=1}^{k} \chi_{\mathcal{D}\left(s+d-2, s, 1_{ \pm}^{2 m}\right)}^{s o(2, d)}+\sum_{m=1}^{k} \chi_{\mathcal{D}\left(d-1,1_{ \pm}^{2 m+1}\right)}^{s o(2, d)}
$$

Notice that contrarily to the case of odd rank, this tensor product contains the tower of totally symmetric fields of arbitrary spin but does not contain the massive scalar $\mathcal{D}(d-1, \mathbf{0})$. The latter is instead part of the tensor product decomposition of two Di singletons of opposite chiralities, together with hook-shaped massless fields whose first columns are of any even height as well as massive $p$-forms with $p=2,4, \ldots, r-1$ :

$$
\chi_{\mathrm{Di}_{+}}^{s o(2, d)} \times \chi_{\mathrm{Di}_{-}}^{s o(2, d)}=\sum_{s=2}^{\infty} \sum_{m=1}^{k} \chi_{\mathcal{D}\left(s+d-2,\left(s, 1^{2 m-1}\right)\right)}^{s o(2, d)}+\sum_{m=0}^{k} \chi_{\mathcal{D}\left(d-1,1^{2 m}\right)}^{s o(2, d)} .
$$

A computation similar to the previous case shows that the tensor product of a spinor singleton of fixed chirality with its anti-singleton can be decomposed as follows:

$$
\sum_{j=0}^{r} \chi_{\mathrm{Di}_{ \pm}}^{s o(2, d)}\left(x_{j}, \boldsymbol{x}_{j}\right) \times \chi_{\overline{\mathrm{Di}}_{ \pm}}^{s o(2, d)}\left(x_{j}, \boldsymbol{x}_{j}\right)=\chi_{0}^{s o(2, d)}\left(x_{0}, \boldsymbol{x}\right)+\sum_{s=2}^{\infty} \sum_{m=0}^{k} \chi_{\left(s-1, s-1,1_{ \pm}^{2 m}\right)}^{s o(2+d)}\left(x_{0}, \boldsymbol{x}\right),
$$

i.e. as the direct sum of the Young diagram describing the Killing tensors associated to each massless field appearing in (3.45). Finally, the tensor product $\mathrm{Di}_{+} \otimes \overline{\mathrm{Di}_{-}}$, as well as the tensor product $\mathrm{Di}_{-} \otimes \overline{\mathrm{Di}_{+}}$, both contain the same $s o(2+d)$ diagrams, i.e. those associated with the Killing tensor of the massless fields appearing in (3.46), namely,

$$
\sum_{j=0}^{r} \chi_{\mathrm{Di}_{ \pm}}^{s o(2, d)}\left(x_{j}, \boldsymbol{x}_{j}\right) \times \chi_{\overline{\mathrm{Di}}_{\mp}}^{s o(2, d)}\left(x_{j}, \boldsymbol{x}_{j}\right)=\sum_{s=2}^{\infty} \sum_{m=0}^{k-1} \chi_{\left(s-1, s-1,1^{2 m+1}\right)}^{s o(2+d)}\left(x_{0}, \boldsymbol{x}\right) .
$$


If one instead consider a spinor singleton which is a Dirac fermion, i.e. contains both chiralities, then the corresponding Di module is given by the direct sum of the two chiral modules:

$$
\text { Di }:=\mathcal{D}\left(\frac{d-1}{2}, \frac{\mathbf{1}}{\mathbf{2}}\right)=\mathcal{D}\left(\frac{d-1}{2}, \frac{\mathbf{1}}{\mathbf{2}}_{+}\right) \oplus \mathcal{D}\left(\frac{d-1}{2}, \frac{\mathbf{1}}{\mathbf{2}}\right),
$$

whose character reads

$$
\chi_{\mathrm{Di}}^{s o(2, d)}(q, \boldsymbol{x})=q^{\frac{d-1}{2}}(1-q)\left(\chi_{\frac{1}{2}_{+}}^{s o(d)}(\boldsymbol{x})+\chi_{\frac{1}{2_{-}}}^{s o(d)}(\boldsymbol{x})\right) \mathcal{P}_{d}(q, \boldsymbol{x}) .
$$

Notice that in this case, the characters of the parity-invariant $\mathrm{Di}$ and $\overline{\mathrm{Di}}$ modules are also simply related by a dimension dependent sign, namely,

$$
\chi_{\overline{\mathrm{Di}}}^{s o(2, d)}(q, \boldsymbol{x})=(-1)^{d+1} \chi_{\mathrm{Di}}^{s o(2, d)}(q, \boldsymbol{x}) .
$$

The endomorphism algebra of this parity-invariant singleton admits a similar decomposition to the previously covered odd- $d$ case, except for the fact that most diagrams have a multiplicity 2 :

$$
\begin{aligned}
\sum_{k=0}^{r} \chi_{\mathrm{Di}}^{s o(2, d)}\left(x_{k}, \boldsymbol{x}_{k}\right) \times \chi_{\overline{\mathrm{Di}}}^{s o(2, d)}\left(x_{k}, \boldsymbol{x}_{k}\right) \\
=2 \chi_{0}^{s o(2+d)}\left(x_{0}, \boldsymbol{x}\right)+2 \sum_{s=2}^{\infty} \sum_{m=0}^{r-1} \chi_{\left(s-1, s-1, \mathbf{1}^{m}\right)}^{s o(2+d)}\left(x_{0}, \boldsymbol{x}\right)+ \\
\quad+\sum_{s=2}^{\infty}\left(\chi_{\left(s-1, s-1, \mathbf{1}_{+}^{r-1}\right)}^{s o(2+d)}\left(x_{0}, \boldsymbol{x}\right)+\chi_{\left(s-1, s-1, \mathbf{1}_{-}^{r-1}\right)}^{s o(2+d)}\left(x_{0}, \boldsymbol{x}\right)\right) .
\end{aligned}
$$

The appearance of those extra degeneracies with respect to the odd- $d$ case (3.30) is caused by the fact that we include both chiralities in (3.48), hence the representations for which the last $s o(d)$ weight vanishes (i.e. $\ell_{r}=0$ ) come twice.

\subsection{Type AB}

Although one of the appealing features of higher-spin holography is the fact that these dualities do not require supersymetry, the four dimensional higher-spin gravity admits a supersymmetric extension with arbitrary $\mathcal{N}$ : see [54] for a review (as well as the recent paper [55] where several one-loop tests of these extensions were performed, together with [26] for the 6-dimensional case). Supersymmetric higher-spin algebras ${ }^{7}$ were studied in four dimensions in [43, 46, 57], an analysis later extended to any dimension in [8], where it was also shown that the spectrum of these supersymmetric higher-spin theories is given by the tensor product of the direct sum of the Rac and Di singletons (possibly decorated with Chan-Paton factor, that we will not consider here).$^{8}$

In four dimensions, the $\mathcal{N}=1$ supersymmetric extension of the algebra $\operatorname{so}(2,3) \cong$ $s p(4, \mathbb{R})$ is the superalgebra $\operatorname{osp}(1 \mid 4)$, of which the sum $\mathrm{Di} \oplus \operatorname{Rac}$ is a supermultiplet. The

\footnotetext{
${ }^{7}$ In dimensions 3,4 and 6, the higher symmetries of super-Laplacians were studied in [56], thereby extending Eastwood's approach to the supersymmetric case.

${ }^{8}$ See also $[26,39,58-60]$ for the quasiconformal approach to higher-spin (super)algebras.
} 
tensor product of a Rac with a Di decomposes into an infinite tower of totally symmetric massless fields of all half-integer spin $s=\frac{1}{2}, \frac{3}{2}, \ldots$, and therefore the tensor product of the irreducible $\operatorname{osp}(1 \mid 4)$-module $\mathrm{Di} \oplus$ Rac with itself contains all totally symmetric fields of integer and half-integer spins (as well as the mixed-symmetry fields appearing in the tensor product of two Di singletons in higher dimensions). The $\mathcal{N}=1$ higher-spin superalgebra extending $\operatorname{osp}(1 \mid 4)$ can be realized in terms of the oscillators $a$ and $b$ introduced in the section 2 by relaxing the constraint of parity. By extending the computations of $s o(2,3)$ characters, one can check at the level of characters the isomorphism [18]:

$$
\operatorname{Adj}=\bigoplus_{s=1, \frac{3}{2}, 2, \frac{5}{2}, \ldots} \mathcal{D}(1-s, s-1)=(\operatorname{Di} \oplus \operatorname{Rac}) \otimes(\overline{\mathrm{Di}} \oplus \overline{\mathrm{Rac}})
$$

which is the supersymmetric extension of (2.48). Here, "Adj" stands for the adjoint module of the four-dimensional $\mathcal{N}=1$ higher-spin superalgebra.

In higher dimensions, the Di and Rac do not form a supermultiplet on their own, due to the fact that the $\mathrm{AdS}_{d+1}$ isometry algebra $s o(2, d)$ admits a supersymmetric extension (i.e. a Lie superalgebra which contains the latter in its bosonic subsector), only in dimensions $d+1=4,5,6$ and 7 , superalgebras which are respectively $\operatorname{osp}(\mathcal{N} \mid 4), \operatorname{sl}(\mathcal{N} \mid 4), F(4)$ and $\operatorname{osp}(\mathcal{N} \mid 8)$. Nevertheless, let us investigate the twisted Flato-Fronsdal theorem for this pair of modules. Using (3.33), the product of the character of a Rac singleton with that of the $\overline{\mathrm{Di}}$ anti-singleton can be written as

$$
\chi_{\operatorname{Rac}}^{s o(2, d)}(q, \boldsymbol{x}) \times \chi_{\overline{\mathrm{Di}}}^{s o(2, d)}(q, \boldsymbol{x})=q^{-\frac{1}{2}}\left(1-q^{2}\right) \mathcal{P}_{d}(q, \boldsymbol{x}) \sum_{s=0}^{\infty} q^{-s} \chi_{\left(s+\frac{1}{2}, \frac{1}{2}^{r-1}\right)}^{s o(\boldsymbol{x})},
$$

whereas for $\overline{\mathrm{Rac}}$ with Di:

$$
\left.\chi_{\overline{\mathrm{Rac}}}^{s o(2, d)}(q, \boldsymbol{x}) \times \chi_{\mathrm{Di}}^{s o(2, d)}(q, \boldsymbol{x})=q^{\frac{1}{2}}\left(1-q^{-2}\right) \mathcal{P}_{d}\left(q^{-1}, \boldsymbol{x}\right) \sum_{s=0}^{\infty} q^{s} \chi_{\left(s+\frac{1}{2}, \frac{1}{2}\right.}^{s o(d)}\right)(\boldsymbol{x}),
$$

Symmetrizing the $r+1$ variables of the above expression and using (3.8), we end up with the following sum of $s o(2+d)$ characters:

$$
\begin{aligned}
& \sum_{k=0}^{r}\left(\chi_{\mathrm{Rac}}^{s o(2, d)}\left(x_{k}, \boldsymbol{x}_{k}\right) \times \chi_{\overline{\mathrm{Di}}}^{s o(2, d)}\left(x_{k}, \boldsymbol{x}_{k}\right)+\chi_{\overline{\mathrm{Rac}}^{s o(2, d)}}\left(x_{k}, \boldsymbol{x}_{k}\right) \times \chi_{\mathrm{Di}}^{s o(2, d)}\left(x_{k}, \boldsymbol{x}_{k}\right)\right) \\
& =\sum_{s=0}^{\infty} \chi_{\left(s+\frac{1}{2}, s+\frac{1}{2}, \frac{1}{2}^{r-1}\right)}^{s o(d+2)}\left(x_{0}, \boldsymbol{x}\right)-\sum_{s=0}^{\infty} \chi_{\left(s-\frac{3}{2}, s+\frac{1}{2}, \frac{1}{2}\right.}^{s o(d+1)}\left(x_{0}, \boldsymbol{x}\right)
\end{aligned}
$$

Using the symmetry property (3.10), the characters appearing in the second sum can be expressed as the characters of bona fide $s o(2+d)$ Young diagrams, given that

$$
\chi_{\left(s-\frac{3}{2}, s+\frac{1}{2}, \frac{1}{2}^{r-1}\right)}^{s o(d+2)}=-\chi_{\left(s-\frac{1}{2}, s-{\frac{1}{2}, \frac{1}{2}^{r-1}}^{s o(d+2)},\right.}^{\chi_{\left(-\frac{1}{2},-\frac{1}{2}, \frac{1}{2}^{r-1}\right)}^{s o(d+2)}}=0
$$


hence we end up with

$$
\begin{aligned}
& \sum_{k=0}^{r} \chi_{\mathrm{Di} \oplus \operatorname{Rac}}^{s o(2, d)}\left(x_{k}, \boldsymbol{x}_{k}\right) \times \chi_{\overline{\mathrm{Di}} \oplus \overline{\operatorname{Rac}}}^{s o(2, d)}\left(x_{k}, \boldsymbol{x}_{k}\right) \\
& =\sum_{s=1}^{\infty} \sum_{m=1}^{r-1} \chi_{\left(s-1, s-1,1^{m}\right)}^{s o(2+d)}\left(x_{0}, \boldsymbol{x}\right)+\sum_{s=1}^{\infty}\left(\chi_{(s-1, s-1)}^{s o(d+2)}\left(x_{0}, \boldsymbol{x}\right)+2 \chi_{\left(s-\frac{1}{2}, s-\frac{1}{2}, \frac{1}{2}^{r-1}\right)}^{s o(d+2)}\left(x_{0}, \boldsymbol{x}\right)\right) .
\end{aligned}
$$

Notice that we are considering a parity-invariant spin- $\frac{1}{2}$ singleton here. Strictly speaking, the formula (3.57) holds for odd $d$. For $d=2 r$, we have $\mathrm{Di}=\mathrm{Di}_{+} \oplus \mathrm{Di}_{-}$, which leads to the appearance of a multiplicity 2 (that we leave implicit) for all diagrams appearing in the above identity, except those of maximal height (namely for $m=r-1$ in the first sum on the left hand side) which appear once with each chirality. Again, this result agrees with the gauge fields present in the type-AB higher-spin gravity.

\section{Lower dimensions}

\subsection{Two dimensions}

Although the $d=1$ case may appear ${ }^{9}$ somewhat degenerate from the point of view of the higher-spin interpretation of its representations, the characters of the conformal algebra $s o(2,1)$ (see e.g. [64-66] for details on the representations of this algebra) provide a useful toy model for seeing explicitly the subtleties related to their domain of convergences.

Relevant modules. The character of an $s o(2,1)$ Verma module $\mathcal{V}_{\Delta}$ of lowest-weight $\Delta$ is

$$
\chi_{\mathcal{V}_{\Delta}}^{s o(2,1)}(q)=\frac{q^{\Delta}}{1-q} \stackrel{|q|<1}{=} \sum_{n=0}^{\infty} q^{\Delta+n}
$$

The domain of convergence of the power series in the variable $q$ around the origin $q=0$, is the disk $|q|<1$. This meromorphic function admits an analytic continuation in the domain $|q|>1$ where it has a convergent power series expansion in the variable $q^{-1}$ around the point at infinity $q=\infty$ :

$$
\chi_{\mathcal{V}_{\Delta}}^{s o(2,1)}(q)=-\chi_{\mathcal{V}_{1-\Delta}}^{s o(2,1)}\left(q^{-1}\right)=-\frac{q^{\Delta-1}}{1-q^{-1}} \stackrel{|q|>1}{=}-\sum_{n=0}^{\infty} q^{\Delta-1-n} .
$$

However, the coefficients of this power series in $q^{-1}$ are negative integers, which prohibit the interpretation of this function as the character of a highest-weight module.

The lowest-weight case (4.1) should be compared with the character of the $s o(2,1)$ Verma module $\overline{\mathcal{V}_{\Delta}}$ of highest-weight $-\Delta$ :

$$
\chi_{\overline{\mathcal{V}_{\Delta}}}^{s o(2,1)}(q)=\chi_{{\mathcal{\nu _ { \Delta }}}^{s o(2,1)}}\left(q^{-1}\right)=\frac{q^{-\Delta}}{1-q^{-1}} \stackrel{|q|>1}{=} \sum_{n=0}^{\infty} q^{-\Delta-n} .
$$

\footnotetext{
${ }^{9}$ Actually, higher-spin extensions of two-dimensional Jackiw-Teitelboim gravity have been considered [61-63] in the context of $\mathrm{AdS}_{2} / \mathrm{CFT}_{1}$ holography.
} 
The domain of convergence of this power series around the point at infinity $q=\infty$ is the domain $|q|>1$. One should stress that it is this power series in $q^{-1}$ with positive integer coefficients that justifies the interpretation of this meromorphic function as the character of a highest-weight module. However, notice the identity,

$$
\chi_{\overline{\mathcal{V}_{\Delta}}}^{s o(2,1)}=-\chi_{\mathcal{V}_{1-\Delta}}^{s o(2,1)}
$$

which is valid everywhere (except at $q=0, \infty$ ).

The Verma module $\mathcal{V}_{\Delta}$ is unitary for $\Delta>0$. For $\Delta \leqslant 0$, the module is non-unitary and becomes reducible for non-positive half-integer $\Delta=-j$ : the non-unitary module $\mathcal{V}_{-j}$ contains an invariant submodule $\mathcal{V}_{j+1}$ which is unitary. The irreducible module $\mathcal{D}_{j}=\mathcal{V}_{-j} / \mathcal{V}_{j+1}$ is nothing but the $(2 j+1)$-dimensional spin- $j$ module, which is non-unitary for $s o(2,1)$ but becomes unitary for $s o(3)$. The character of the irreducible module $\mathcal{D}_{j}$ is

$$
\begin{aligned}
\chi_{\mathcal{D}_{j}}^{s o(2,1)}(q) & =\chi_{\mathcal{V}_{-j}}^{s o(2,1)}(q)-\chi_{\mathcal{V}_{j+1}}^{s o(2,1)}(q)=\sum_{n=-j}^{j} q^{n} \\
& =\frac{q^{j+\frac{1}{2}}-q^{-j-\frac{1}{2}}}{q^{\frac{1}{2}}-q^{-\frac{1}{2}}}=\frac{\sinh \left(j+\frac{1}{2}\right) \beta}{\sinh \frac{\beta}{2}} \equiv \chi_{j}^{s o(3)}(i \beta) .
\end{aligned}
$$

Since this $s o(2,1)$-module is finite-dimensional, it is both lowest-weight and highest-weight, which translates into the property $\chi_{\mathcal{D}_{j}}^{\text {so(2,1) }}\left(q^{-1}\right)=\chi_{\mathcal{D}_{j}}^{\text {so(2,1) }}(q)$. The Laurent series in the right of (4.5) contains negative powers, seen either as a power series in $q$ or as a power series in $q^{-1}$, but it converges for any $q \neq 0, \infty$.

Flato-Fronsdal theorem. The Clebsch-Gordan decomposition of these finite-dimensional so $(2,1)$-modules is the celebrated rule

$$
\chi_{\mathcal{D}_{j_{1}}}^{s o(2,1)}(q) \times \chi_{\mathcal{D}_{j_{2}}}^{s o(2,1)}(q)=\sum_{j=\left|j_{1}-j_{2}\right|}^{j_{1}+j_{2}} \chi_{\mathcal{D}_{j}}^{s o(2,1)}(q)
$$

as can be checked by an explicit computation of the product of characters of the form (4.5). One may also consider the tensor product of two lowest-weight Verma modules,

$$
\chi_{\mathcal{V}_{\Delta_{1}}}^{s o(2,1)}(q) \times \chi_{\mathcal{V}_{\Delta_{2}}}^{s o(2,1)}(q)=\frac{q^{\Delta_{1}+\Delta_{2}}}{(1-q)^{2}} \stackrel{|q|<1}{=} \sum_{n=0}^{\infty} \chi_{\mathcal{V}_{\Delta_{1}+\Delta_{2}+n}}^{s o(2,1)}(q)
$$

where we used the formula (4.1) and expanded $(1-q)^{-1}$ in power series around the origin (valid for $|q|<1$ ) to obtain the result,

$$
\mathcal{V}_{\Delta_{1}} \otimes \mathcal{V}_{\Delta_{2}}=\bigoplus_{n=0}^{\infty} \mathcal{V}_{\Delta_{1}+\Delta_{2}+n}
$$

which we will use later on.

The formula (4.9) is a sort of $d=1$ analogue of the Flato-Fronsdal theorem, in the sense that it is a decomposition of the tensor product of two lowest-weight infinite-dimensional 
modules. However, it becomes much more delicate to manipulate the tensor product of infinite-dimensional modules where one module is lowest-weight and the other one is highest-weight (as in the twisted Flato-Fronsdal theorem) because the resulting module is neither lowest nor highest weight. Therefore, its character involves Laurent series which should be treated with care.

Twisted Flato-Fronsdal theorem. Treating the characters as meromorphic functions and performing the power series expansion, one can write the equality as

$$
\begin{gathered}
\chi_{\mathcal{V}_{\Delta_{1}}}^{s o(2,1)}(q) \times \chi_{\overline{\mathcal{V}}_{\Delta_{2}}}^{s o(2,1)}(q)= \\
\stackrel{|q|<1}{=}-\chi_{\mathcal{V}_{\Delta_{1}}}^{s o(2,1)}(q) \times \chi_{\mathcal{V}_{1-\Delta_{2}}}^{s o(2,1)}(q)=\frac{-q^{1+\Delta_{1}-\Delta_{2}}}{(1-q)^{2}} \chi_{\mathcal{V}_{1+\Delta_{1}-\Delta_{2}+n}}^{s o(2,1)}(q) .
\end{gathered}
$$

Unfortunately, the last line has negative coefficients as a power series (in $q$ or in $q^{-1}$ ), which confirms that it lacks any sound group-theoretical intepretation as lowest or highest weight module.

In fact, the group-theoretical interpretation of the factors in the left-hand-side of (4.10), as characters of lowest vs highest weight modules, holds in two distinct domains $(|q|<1$ versus $|q|>1)$. The same remark holds for the infinite sum $\bigoplus_{j=0}^{\infty} \mathcal{D}_{j}$ of all irreducible finite-dimensional so $(2,1)$ modules, which could be considered as a $d=1$ analogue of the adjoint module: it is tempting (and will be justified below) to compute the formal sum $\sum_{j=0}^{\infty} \chi_{\mathcal{D}_{j}}^{s o(2,1)}(q)$ via the formula (4.5) as the difference of the two series in the right-handsides of

$$
\sum_{j=0}^{\infty} \chi_{\mathcal{V}_{-j}}^{s o(2,1)}(q) \stackrel{|q|<1}{=} \frac{-q}{(1-q)^{2}} \quad \text { and } \quad \sum_{j=0}^{\infty} \chi_{\mathcal{V}_{j+1}}^{s o(2,1)}(q) \stackrel{|q|>1}{=} \frac{q}{(1-q)^{2}},
$$

to obtain the meromorphic function

$$
\sum_{j=0}^{\infty} \chi_{\mathcal{D}_{j}}^{s o(2,1)}(q)=-\frac{1}{2 \sinh ^{2} \frac{\beta}{2}}=-\frac{2 q}{(1-q)^{2}},
$$

which has negative coefficients as a power series, whether in $q$ or $q^{-1}$. Again, this fact precludes any clear group-theoretical interpretation. Nevertheless, the equality (4.12) is valid. Indeed, the following trigonometric series is divergent but can be evaluated via Cesaro's resummation: ${ }^{10}$

$$
\sum_{n=0}^{\infty} \sin \left[\left(n+\frac{1}{2}\right) x\right]=\frac{1}{2 \sin \frac{x}{2}},
$$

from which one deduces the character formula,

$$
\sum_{j=0}^{\infty} \chi_{j}^{s o(3)}(\alpha)=\frac{1}{2 \sin ^{2} \frac{\alpha}{2}},
$$

\footnotetext{
${ }^{10}$ See e.g. [50] (chapter XIII, section 60, ex. 7).
} 
which holds as a distribution and whose Wick rotation is (4.12). Therefore, one can write the relation,

$$
\sum_{j=0}^{\infty} \chi_{\mathcal{D}_{j}}^{s o(2,1)}(q)=2 \chi_{\mathcal{V}_{\Delta}}^{s o(2,1)}(q) \times \chi_{\overline{\mathcal{V}_{\Delta}}}^{s o(2,1)}(q)
$$

as a $d=1$ analogue of the twisted Flato-Fronsdal theorem. ${ }^{11}$

In order to provide a concrete realization of the isomorphism,

$$
\mathcal{V}_{\Delta} \otimes \overline{\mathcal{V}_{\Delta}}=\bigoplus_{j=0}^{\infty} \mathcal{D}_{j}
$$

let us consider the elements $L_{+}^{m}|\Delta\rangle$ of the Verma module $\mathcal{V}_{\Delta}$ of $s o(2,1)$ generated by the lowest-weight vector $|\Delta\rangle$. Mimicking the discussion of the oscillator realization in section 2, one can introduce two concrete realizations of the generators of $\operatorname{End}\left(\mathcal{V}_{\Delta}\right)$, and compare the so $(2,1)$ decomposition in the two cases:

- Firstly, as elements of $\mathcal{V}_{\Delta} \otimes \overline{\mathcal{V}_{\Delta}}$,

$$
T^{m ; n}:=L_{+}^{m}|\Delta\rangle\langle\Delta| L_{-}^{n} .
$$

with $m, n \in \mathbb{N}$. The identities

$$
\begin{aligned}
& {\left[L_{-}, T^{m ; n}\right]=m(2 \Delta+m-1) T^{m-1 ; n}-T^{m ; n+1},} \\
& {\left[L_{+}, T^{m ; n}\right]=T^{m+1 ; n}-n(2 \Delta+n-1) T^{m ; n-1} .}
\end{aligned}
$$

allows to identify the lowest (or highest) weight states of the spin- $j$ submodule $\mathcal{D}_{j} \subset \operatorname{End}\left(\mathcal{V}_{\Delta}\right):$ they are the elements

$$
Y_{-j}^{j}:=\sum_{m=0}^{\infty} \frac{\Gamma(2 \Delta-1)}{m ! \Gamma(2 \Delta+m)} T^{m ; m+j}, \quad Y_{j}^{j}:=\sum_{m=0}^{\infty} \frac{\Gamma(2 \Delta-1)}{m ! \Gamma(2 \Delta+m)} T^{m+j ; m}
$$

of $\mathcal{V}_{\Delta} \otimes \overline{\mathcal{V}_{\Delta}}$

- Secondly, as elements in the universal enveloping algebra ${ }^{12} \mathcal{U}(s o(2,1))$. Let us recall that the Lie algebra $s o(2,1)$ is spanned by the generators $\left\{E, L_{+}, L_{-}\right\}$obeying the commutation relations

$$
\left[E, L_{ \pm}\right]= \pm L_{ \pm}, \quad\left[L_{-}, L_{+}\right]=2 E
$$

\footnotetext{
${ }^{11}$ The tensor product of two discrete series representations of respectively highest and lowest weight kind has been studied in [67] (see [66] for a recent review): its usual decomposition contains in fact principal as well as complementary series representations on top of discrete series ones. Therefore, naively the tensor product decomposition of [67] is not reproduced by our so $(2,1)$ character analysis. However, these two approaches are difficult to compare because continuous (principal and complementary) series representations have unbounded spectrum of $E$. Nevertheless, the decomposition (4.15) will be justified below.

${ }^{12}$ This is motivated by the fact that there exists a natural inclusion $\mathcal{U}(\mathfrak{g}) / \operatorname{Ann}(M) \hookrightarrow \operatorname{End}(M)$ for $\mathfrak{g}$ a Lie algebra and $M$ a g-modules with annihilator $\operatorname{Ann}(M)$ [68].
} 
Following closely the presentation of the universal enveloping algebra of $s o(2,1) \cong$ $\operatorname{sl}(2, \mathbb{R})[69,70]$ (see also $[71,72]$ for more details), we can consider the basis given by

$$
X_{m}^{j, k}:=c_{j, m} \mathcal{C}_{2}{ }^{k} \operatorname{ad}_{L_{-}}^{m} L_{+}^{j}, \quad \text { with } \quad 0 \leqslant m \leqslant 2 j, \quad k \in \mathbb{N},
$$

and where $\mathcal{C}_{2}:=E^{2}-\frac{1}{2}\left(L_{+} L_{-}+L_{-} L_{+}\right)=E(E-1)+L_{+} L_{-}$is the Casimir operator of $s o(2,1)$ generating the center of the universal enveloping algebra, while the coefficients $c_{j, m}$ are normalization factors. The decomposition of $\mathcal{U}(s o(2,1))$ in terms of finitedimensional modules under the adjoint action of $s o(2,1)$ can be read more easily, as

$$
\operatorname{ad}_{L_{+}} L_{+}^{j}=0, \quad \text { and } \quad \operatorname{ad}_{E} L_{+}^{j}=j L_{+}^{j}, \quad \forall j \in \mathbb{N},
$$

i.e. $L_{+}^{j}$ defines a highest-weight vector of weight $j$, and thus the various power of $\operatorname{ad}_{L_{-}}^{m}$ for $m=0, \ldots, 2 j$ define the elements of this spin- $j$ module. However, each of those modules have an infinite multiplicity in $\mathcal{U}(s o(2,1))$ since they appear dressed with arbitrary power of the quadratic Casimir operator of $s o(2,1)$ according to (4.21). Considering the quotient,

$$
g l[\lambda]:=\frac{\mathcal{U}(s o(2,1))}{\left\langle\mathcal{C}_{2}-\mu_{\lambda} \mathbb{1}\right\rangle}, \quad \text { with } \quad \mu_{\lambda}:=\frac{\lambda^{2}-1}{4},
$$

i.e. by modding out the ideal $\mathcal{I}_{\lambda}=\left(\mathcal{C}_{2}-\mu_{\lambda} \mathbb{1}\right) \mathcal{U}(s o(2,1))$ of the universal enveloping algebra, the vector space of the resulting algebra decomposes as the direct sum [73]

$$
g l[\lambda]=\bigoplus_{j=0}^{\infty} \mathcal{D}_{j} .
$$

In other word, by fixing the value of the Casimir operator, one lifts the (infinite) degeneracy of the finite-dimensional modules. A basis of $g l[\lambda]$ is therefore given by:

$$
V_{n}^{j}:=(-1)^{j+n} \frac{(j+n) !}{(2 j) !} \operatorname{ad}_{L_{-}}^{j-n} L_{+}^{j}, \quad \text { with } \quad|n| \leqslant j,
$$

where the generators $\left\{V_{n}^{j}\right\}_{-j \leqslant n \leqslant j}$ span the spin $j$ module $\mathcal{D}_{j}$ in the decomposition (4.24). One can also describe this decomposition in more covariant terms by making use of the generators $\tilde{L}^{A}:=\frac{1}{2} \epsilon^{A B C} L_{B C}$ of $s o(2,1)$. All elements of $\mathcal{U}(s o(2,1))$ can be written as linear combinations of elements of the form $P\left(\mathcal{C}_{2}\right) \tilde{L}_{\left\{A_{1}\right.} \cdots \tilde{L}_{\left.A_{j}\right\}}$ where $P\left(\mathcal{C}_{2}\right)$ is a polynomial in the quadratic Casimir $\mathcal{C}_{2}=-\tilde{L}^{A} \tilde{L}_{A}$ and the brackets over the indices indicates total symmetrization over all indices and traceless projection. Therefore, $\mathcal{U}(s o(2,1))$ branches in spin- $j$ submodules. Moreover, the quadratic Casimir operator takes the value $\mathcal{C}_{2}=\Delta(\Delta-1)$ on the Verma module $\mathcal{V}_{\Delta}$ with $\Delta=\frac{1 \pm \lambda}{2}$. Therefore, the elements $\tilde{L}_{\left\{A_{1}\right.} \cdots \tilde{L}_{\left.A_{j}\right\}}$ provide a covariant basis of $\operatorname{End}\left(\mathcal{V}_{\Delta}\right)$.

\subsection{Three dimensions}

The $d=2$ conformal algebra is a direct sum of two $d=1$ conformal algebras: $s o(2,2)=$ $s o(2,1) \oplus s o(2,1)$. Accordingly, the $s o(2,2)$ Verma module $\mathcal{V}(\Delta, s)$ is related to the that of $s o(2,1)$ as

$$
\mathcal{V}(\Delta, s)=\mathcal{V}_{\frac{\Delta+s}{2}} \otimes \mathcal{V}_{\frac{\Delta-s}{2}}
$$


Note that the spin $s$ can take negative values here since they are eigenvalues of $s o(2)$. Introducing the variables,

$$
z=q x=e^{-\beta+i \alpha}, \quad \bar{z}=q x^{-1}=e^{-\beta-i \alpha},
$$

for the $s o(2,2)$ weights, the character of $\mathcal{V}(\Delta, s)$ is given by

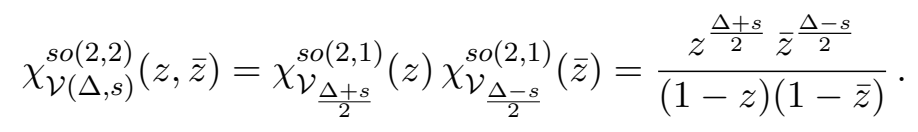

Relevant modules. The character of a scalar field of lowest energy $E_{0}=\Delta$ is

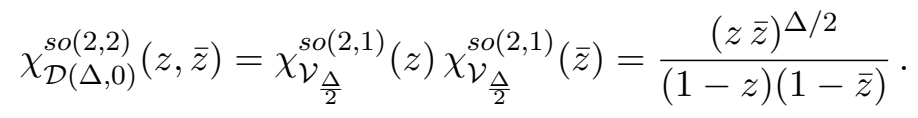

In the limit when the conformal weight of the scalar field goes to the unitarity bound, $\Delta \rightarrow \frac{d-2}{2}=0$, one finds

$$
\chi_{\mathcal{D}(\Delta \rightarrow 0,0)}^{s o(2,2)}(z, \bar{z})=\frac{1}{(1-z)(1-\bar{z})}=1+\frac{z}{1-z}+\frac{\bar{z}}{1-\bar{z}}+\chi_{\mathcal{D}(2,0)}^{s o(2,2)}(z, \bar{z}),
$$

which can be understood from the property of the $s o(2,1)$-module,

$$
\mathcal{V}_{0}=1 \oplus \mathcal{V}_{1}
$$

where 1 denotes the trivial representation of $s o(2,1)$. The last term in (4.30) reflects the appearance of a submodule, $\mathcal{D}(2,0) \subset \mathcal{V}(0,0)$. The appearance of such a submodule holds in any dimension: $\mathcal{D}\left(\frac{d+2}{2}, \mathbf{0}\right) \subset \mathcal{V}\left(\frac{d-2}{2}, \mathbf{0}\right)$, and the Rac has been defined uniformly for all dimensions as the quotient $\mathcal{V}\left(\frac{d-2}{2}, \mathbf{0}\right) / \mathcal{D}\left(\frac{d+2}{2}, \mathbf{0}\right)$, cf $(3.12)$. However, in $d=2$ the $\operatorname{Rac}=\mathcal{V}(0,0) / \mathcal{D}(2,0)$ is a reducible module: its character reads

$$
\chi_{\operatorname{Rac}}^{s o(2,2)}(z, \bar{z})=1+\frac{z}{1-z}+\frac{\bar{z}}{1-\bar{z}},
$$

where each of the three terms correspond to the characters of different irreducible modules. The first term is the character of the trivial module, $\mathcal{D}(0,0)$, which corresponds to a zeromode in field-theoretical terms. In fact, the Rac always describes a conformal scalar in dimension $d$ but the zero-mode has canonical conformal weight (i.e. $\frac{d-2}{2}=0$ ) only for $d=2$. The last two terms are the characters of the modules $\mathcal{D}(1,+1)$ and $\mathcal{D}(1,-1)$, which correspond to the left and right moving scalar fields living on the $d=2$ conformal boundary, respectively.

Let us introduce the notations,

$$
\mathcal{V}(\Delta, s)_{0}:=\mathcal{V}(\Delta,+s) \oplus \mathcal{V}(\Delta,-s), \quad \text { and } \quad \mathcal{D}(\Delta, s)_{0}:=\mathcal{D}(\Delta,+s) \oplus \mathcal{D}(\Delta,-s),
$$

for parity-invariant combinations. The Di module, describing the $d=2$ conformal spinor, is $\mathrm{Di}=\mathcal{D}\left(\frac{1}{2}, \frac{1}{2}\right)_{0}=\mathrm{Di}_{+} \oplus \mathrm{Di}_{-}$with $\mathrm{Di}_{ \pm}=\mathcal{D}\left(\frac{1}{2}, \pm \frac{1}{2}\right)$, cf (3.31) for $d=2$. For spin $s \geqslant 1$, the irreducible module $\mathcal{D}(s, \pm s)$ is given by

$$
\begin{aligned}
& \mathcal{D}(s,+s)=\mathcal{V}(s, s) / \mathcal{V}(s+1, s-1)=\left(\mathcal{V}_{s} \otimes \mathcal{V}_{0}\right) /\left(\mathcal{V}_{s} \otimes \mathcal{V}_{1}\right)=\mathcal{V}_{s} \otimes 1 \\
& \mathcal{D}(s,-s)=\mathcal{V}(s,-s) / \mathcal{V}(s+1,-s+1)=\left(\mathcal{V}_{0} \otimes \mathcal{V}_{s}\right) /\left(\mathcal{V}_{1} \otimes \mathcal{V}_{s}\right)=1 \otimes \mathcal{V}_{s}
\end{aligned}
$$




\begin{tabular}{|c|c|c|c|}
\hline Modules & AdS $_{3}$ & CFT $_{2}$ & Equivalent descriptions \\
\hline $\mathcal{D}(0,0)$ & Vacuum & Constant zero-mode & $1 \otimes 1$ \\
\hline $\mathcal{D}(0,0) \Subset \mathcal{D}(1,1)_{0}$ & Rac & Conformal scalar & $\mathcal{V}(0,0) / \mathcal{D}(2,0)$ \\
\hline $\mathcal{D}\left(\frac{1}{2}, \frac{1}{2}\right)_{0}$ & $\mathrm{Di}$ & Conformal spinor & $\left(\mathcal{V}_{\frac{1}{2}} \otimes 1\right) \oplus\left(1 \otimes \mathcal{V}_{\frac{1}{2}}\right)$ \\
\hline $\mathcal{D}(1,1)_{0}$ & $\mathrm{U}(1)^{\otimes 2}$ Chern-Simons & Chiral bosons & $\left(\mathcal{V}_{1} \otimes 1\right) \oplus\left(1 \otimes \mathcal{V}_{1}\right)$ \\
\hline $\mathcal{D}(1,1)_{0} \Subset \mathcal{D}(2,0)$ & Maxwell field & Conserved current & $\mathcal{V}(1,1)_{0} / \mathcal{D}(2,0)$ \\
\hline $\mathcal{D}(s, s)_{0}$ & Massless spin- $s$ field & Conserved spin- $s$ current & $\left(\mathcal{V}_{s} \otimes 1\right) \oplus\left(1 \otimes \mathcal{V}_{s}\right)$ \\
\hline $\mathcal{D}(1-s, s-1)$ & Killing tensor & Conformal Killing tensor & $\left(\mathcal{D}_{s-1} \otimes 1\right) \oplus\left(1 \otimes \mathcal{D}_{s-1}\right)$ \\
\hline
\end{tabular}

Table 1. List of relevant $s o(2,2)$ modules and their field-theoretical interpretations.

The corresponding character reads

$$
\chi_{\mathcal{D}(s,+s)}^{s o(2,2)}(z, \bar{z})=\frac{z^{s}}{1-z}, \quad \chi_{\mathcal{D}(s,-s)}^{s o(2,2)}(z, \bar{z})=\frac{\bar{z}^{s}}{1-\bar{z}} .
$$

For $s \geqslant 1$, the parity-invariant module $\mathcal{D}(s, s)_{0}=\mathcal{V}(s, s)_{0} / \mathcal{V}(s+1, s-1)_{0}$ describes a conserved spin- $s$ conformal current in $d=2$ dimensions or, equivalently, a massless spin$s \mathrm{AdS}_{3}$ field. Their holomorphic decomposition as a direct sum, $\mathcal{D}(s,+s) \oplus \mathcal{D}(s,-s)$, reflects the standard lore that massless fields do not have propagating degrees of freedom in three dimensions. In this sense one may consider all these fields as spin- $s$ singletons (as pointed out in e.g. $[8,74])$. For $s=1$, there is a subtlety in the interpretation: the parityinvariant combination of the two irreducible modules, $\mathcal{D}(1,1)_{0}=\mathcal{D}(1,+1) \oplus \mathcal{D}(1,-1)$, can be obtained as the quotient $\mathcal{V}(1,1)_{0} /(2 \mathcal{D}(2,0))$, whereas the quotient

$$
\operatorname{Max}:=\mathcal{V}(1,1)_{0} / \mathcal{D}(2,0),
$$

which would be the analogue of the higher-dimensional case of spin-1 massless field, is isomorphic to the reducible module $\mathcal{D}(1,1)_{0} \in \mathcal{D}(2,0) .{ }^{13}$ The former module corresponds to the $\mathrm{U}(1) \times \mathrm{U}(1)$ Chern-Simons theory whereas the latter module corresponds to the Maxwell theory. The latter is Hodge dual to a massless scalar in three dimensions without zero-mode. In group-theoretical terms, this equivalence translates into the isomorphisms

$$
\mathcal{V}(0,0)=\mathcal{D}(0,0) \oplus \operatorname{Max}=\operatorname{Rac} \oplus \mathcal{D}(2,0),
$$

since $\operatorname{Max}=\mathcal{D}(1,1)_{0} \notin \mathcal{D}(2,0)$ and $\operatorname{Rac}=\mathcal{D}(0,0) \Subset \mathcal{D}(1,1)_{0}$.

The list of the relevant parity-invariant $s o(2,2)$ modules and their field-theoretical interpretations are summarized in table 1 . Note that we also included the finite-dimensional irreducible modules $\mathcal{D}(1-s, s-1)=\left(\mathcal{D}_{s-1} \otimes 1\right) \oplus\left(1 \otimes \mathcal{D}_{s-1}\right)$ describing conformal Killing tensors of rank $s-1$.

Flato-Fronsdal theorem. Given the identities,

$$
\sum_{s=2}^{\infty} \chi_{\mathcal{D}(s, s)_{0}}^{s o(2,2)}(z, \bar{z})=\left(\frac{z}{1-z}\right)^{2}+\left(\frac{\bar{z}}{1-\bar{z}}\right)^{2},
$$

\footnotetext{
${ }^{13}$ The notation $U=V \Subset W$ indicates thats $U$ is the semidirect sum of the modules $V$ and $W$ thereby indicating that the $W$ is a submodule of $U$ and that $V=U / W$.
} 
and

$$
\sum_{s=1}^{\infty} \chi_{\mathcal{D}(s, s)_{0}}^{s o(2,2)}(z, \bar{z})=\left(\frac{z^{\frac{1}{2}}}{1-z}\right)^{2}+\left(\frac{\bar{z}^{\frac{1}{2}}}{1-\bar{z}}\right)^{2},
$$

it is tempting to write the Flato-Fronsdal theorem for $s o(2,2)$ as

$$
\left(\chi_{\mathcal{D}(1,1)_{0}}^{s o(2,2)}(z, \bar{z})\right)^{2}=2 \chi_{\mathcal{D}(2,0)}^{s o(2,2)}(z, \bar{z})+\sum_{s=2}^{\infty} \chi_{\mathcal{D}(s, s)_{0}}^{s o(2,2)}(z, \bar{z})
$$

and

$$
\left(\chi_{\mathrm{Di}}^{s o(2,2)}(z, \bar{z})\right)^{2}=2 \chi_{\mathcal{D}(1,0)}^{s o(2,2)}(z, \bar{z})+\sum_{s=1}^{\infty} \chi_{\mathcal{D}(s, s)_{0}}^{s o(2,2)}(z, \bar{z}) .
$$

The equation (4.41) is consistent with the generalized Flato-Fronsdal theorem in general dimensions [8], while the case (4.40) is consistent with the tensor product of spin- 1 singleton, namely the type-C case, in general dimensions [11]. In fact, the Flato-Fronsdal theorem in the scalar case is rather

$$
\begin{aligned}
\left(\chi_{\operatorname{Rac}}^{s o(2,2)}(z, \bar{z})\right)^{2}= & {\left[1+\chi_{\mathcal{D}(1,1)_{0}}^{s o(2,2)}(z, \bar{z})+\chi_{\mathcal{D}(2,0)}^{s o(2,2)}(z, \bar{z})\right]+\left[\chi_{\mathcal{D}(1,1)_{0}}^{s o(2,2)}(z, \bar{z})+\chi_{\mathcal{D}(2,0)}^{s o(2,2)}(z, \bar{z})\right] } \\
& +\sum_{s=2}^{\infty} \chi_{\mathcal{D}(s, s)_{0}}^{s o(2,2)}(z, \bar{z}),
\end{aligned}
$$

where the scalar field (i.e. the first term between squared brackets) is described by the reducible module $\mathcal{V}(0,0)$ and contains a non-normalizable zero-mode, and the spin- 1 field (i.e. the second term between squared brackets) corresponds to Maxwell theory. This version of Flato-Fronsdal theorem has been considered in [22] where the IR divergence caused by the zero-mode has been thrown away.

Let us consider now the tensor product of two singletons of spin $s$ and $s^{\prime}$. For the same chiralities, the $d=1$ formula (4.8) implies that the tensor product decomposes into the direct sum of all massless fields of spin $\sigma \geqslant s+s^{\prime}$ and of chirality \pm as

$$
\chi_{\mathcal{D}(s, \pm s)}^{s o(2,2)} \chi_{\mathcal{D}\left(s^{\prime}, \pm s^{\prime}\right)}^{s o(2,2)}=\sum_{\sigma=s+s^{\prime}}^{\infty} \chi_{\mathcal{D}(\sigma, \pm \sigma)}^{s o(2,2)} .
$$

For the opposite chiralities, the tensor product reduces to a single massive field of spin $s-s^{\prime}$ and of minimal energy $s+s^{\prime}$ :

$$
\chi_{\mathcal{D}(s,+s)}^{s o(2,2)} \chi_{\mathcal{D}\left(s^{\prime},-s^{\prime}\right)}^{s o(2,2)}=\chi_{\mathcal{D}\left(s+s^{\prime}, s-s^{\prime}\right)}^{s o} .
$$

Collecting the previous decompositions, we can write the tensor product of two parityinvariant spin- $s$ and spin- $s^{\prime}$ singletons, for $s \neq s^{\prime}$ as

$$
\chi_{\mathcal{D}(s, s)_{0}}^{s o(2,2)}(z, \bar{z}) \chi_{\mathcal{D}\left(s^{\prime}, s^{\prime}\right)_{0}}^{s o(2,2)}(z, \bar{z})=\chi_{\mathcal{D}\left(s+s^{\prime},\left|s-s^{\prime}\right|\right)_{0}}^{s o(2,2)}(z, \bar{z})+\sum_{\sigma=s+s^{\prime}}^{\infty} \chi_{\mathcal{D}(\sigma, \sigma)_{0}}^{s o(2,2)}(z, \bar{z}),
$$

and for $s=s^{\prime} \neq 0$ as

$$
\left(\chi_{\mathcal{D}(s, s)_{0}}^{s o(2,2)}(z, \bar{z})\right)^{2}=2 \chi_{\mathcal{D}(2 s, 0)}^{s o(2,2)}(z, \bar{z})+\sum_{\sigma=2 s}^{\infty} \chi_{\mathcal{D}(\sigma, \sigma)_{0}}^{s o(2,2)}(z, \bar{z}),
$$

which, in particular, reproduces the results for the Di in (4.41) and for the spin-1 singleton in (4.40). 
Twisted Flato-Fronsdal theorem. The finite-dimensional irreps of so(4) are characterised by weights $\left(s_{1}, s_{2}\right)$ where $s_{1}$ and $\left|s_{2}\right|$ are both non-negative integers (or both half-integers) but $s_{2}$ can be negative. It is isomorphic to the tensor product of two so(3) finite dimensional modules, $\mathcal{D}_{\frac{s_{1}+s_{2}}{2}} \otimes \mathcal{D}_{\frac{s_{1}-s_{2}}{2}}$. In terms of the characters, this is

$$
\chi_{\left(s_{1}, s_{2}\right)}^{s o(4)}(q, x)=\chi_{\frac{s_{1}+s_{2}}{2}}^{s o(3)}(z) \chi_{\frac{s_{1}-s_{2}}{2}}^{s o(3)}(\bar{z}),
$$

and in particular,

$$
\chi_{(s-1, s-1)}^{s o(4)}(q, x)=\chi_{s-1}^{s o(3)}(z), \quad \chi_{(s-1,1-s)}^{s o(4)}(q, x)=\chi_{s-1}^{s o(3)}(\bar{z}) .
$$

The characters of the chiral spin- $s$ anti-singletons read

$$
\chi_{\overline{\mathcal{D}(s,+s)}}^{s o(2,2)}(z)=\chi_{\mathcal{D}(s,+s)}^{s o(2,2)}\left(z^{-1}\right), \quad \text { and } \quad \chi_{\overline{\mathcal{D}(s,-s)}}^{s o(2,2)}(\bar{z})=\chi_{\mathcal{D}(s,-s)}^{s o(2,2)}\left(\bar{z}^{-1}\right) .
$$

The formula (4.15) implies the identities:

$$
\begin{aligned}
& 2 \chi_{\mathcal{D}(s,+s)}^{s o(2,2)}(z) \chi_{\mathcal{D}(s,+s)}^{s o(2,2)}(z)=\sum_{\sigma=1}^{\infty} \chi_{(\sigma-1, \sigma-1)}^{s o(4)}(z), \\
& 2 \chi_{\mathcal{D}(s,-s)}^{s o(2,2)}(\bar{z}) \chi_{\overline{\mathcal{D}(s,-s)}}^{s o(2,2)}(\bar{z})=\sum_{\sigma=1}^{\infty} \chi_{(\sigma-1,1-\sigma)}^{s o(4)}(\bar{z}),
\end{aligned}
$$

which can be seen as the twisted Flato-Fronsdal in the chiral (or antichiral) sector. Notice however that the massless fields having the Killing tensors $\mathcal{D}(\sigma-1, \pm(\sigma-1))$ with $\sigma=1, \ldots, 2 s-1$ are not present in the field content given by the Flato-Fronsdal theorem (4.46). In fact, these additional modules can be factorized as

$$
\sum_{\sigma=1}^{2 s-1} \chi_{(\sigma-1, \pm(\sigma-1))}^{s o(4)}=\left(\chi_{(s-1, \pm(s-1))}^{s o(4)}\right)^{2}=\chi_{(s-1, \pm(s-1))}^{s o(4)} \chi_{(s-1, \pm(s-1))}^{s o(4)},
$$

and can be interpreted as the endomorphism algebra of the finite-dimensional module $\mathcal{D}(s-1, s-1)=\mathcal{D}_{s-1} \otimes 1$ (or, respectively, $\mathcal{D}(s-1,1-s)=1 \otimes \mathcal{D}_{s-1}$ ). This reflects that the massless spin- $s$ module $\mathcal{D}(s, s)=\mathcal{V}_{s} \otimes 1$ appears as a submodule of $\mathcal{V}(1-s, 1-s)=\mathcal{V}_{1-s} \otimes 1$, whose irreducible part corresponds to $\mathcal{D}_{s-1}=\mathcal{V}_{1-s} / \mathcal{V}_{s}$. The appearance of the submodule should be related to the fact that the spin- $s$ singletons (for $s \geqslant 1$ ) possess gauge symmetries. We will see in the next section that a similar phenomenon takes place for higher-spin singletons in $d+1=5$ dimensions.

Let us now consider the twisted Flato-Fronsdal theorem for the type-A and type-B models. We note first that the Rac and Di characters satisfy

$$
\chi_{\overline{\mathrm{Rac}} / \overline{\mathrm{Di}}}^{s o(2,2)}(z, \bar{z})=\chi_{\mathrm{Rac} / \mathrm{Di}}^{s o(2,2)}\left(z^{-1}, \bar{z}^{-1}\right)=-\chi_{\mathrm{Rac} / \mathrm{Di}}^{s o(2,2)}(z, \bar{z}) .
$$

For the Di module, the above is due to the symmetry property of $\chi_{\mathcal{D}\left(\frac{1}{2}, \pm \frac{1}{2}\right)}^{s o(2,2)}$. For the Rac module, this is possible only when we include the zero-mode. By taking the product of 
singleton and anti-singleton characters, we obtain

$$
\begin{aligned}
& \chi_{\mathrm{Rac}}^{s o(2,2)}(z, \bar{z}) \chi_{\mathrm{Rac}}^{s o(2,2)}(z, \bar{z})=-\left(1+\frac{z}{1-z}+\frac{\bar{z}}{1-\bar{z}}\right)^{2}, \\
& \chi_{\mathrm{Di}}^{s o(2,2)}(z, \bar{z}) \chi_{\overline{\mathrm{Di}}}^{s o(2,2)}(z, \bar{z})=-\left(\frac{z^{\frac{1}{2}}}{1-z}+\frac{\bar{z}^{\frac{1}{2}}}{1-\bar{z}}\right)^{2} .
\end{aligned}
$$

Analogously to the higher dimensional cases, we take the symmetrization prescription. The exchange $q \leftrightarrow x$ translates into $(z, \bar{z}) \leftrightarrow(z, 1 / \bar{z})$ according to the definition (4.27). Explicit computation leads to

$$
\begin{aligned}
\chi_{\mathrm{Rac}}(z, \bar{z}) \chi_{\overline{\mathrm{Rac}}}(z, \bar{z})+\chi_{\mathrm{Rac}}\left(z, \bar{z}^{-1}\right) \chi_{\overline{\mathrm{Rac}}}\left(z, \bar{z}^{-1}\right) & =-1-\frac{2 z}{(1-z)^{2}}-\frac{2 \bar{z}}{(1-\bar{z})^{2}}, \\
\chi_{\mathrm{Di}}(z, \bar{z}) \chi_{\overline{\mathrm{Di}}}(z, \bar{z})+\chi_{\mathrm{Di}}\left(z, \bar{z}^{-1}\right) \chi_{\overline{\mathrm{Di}}}\left(z, \bar{z}^{-1}\right) & =-\frac{2 z}{(1-z)^{2}}-\frac{2 \bar{z}}{(1-\bar{z})^{2}},
\end{aligned}
$$

where we suppressed the superscript so $(2,2)$ for compactness of the expressions. Comparing these results with the $s o(4)$ characters, we find

$$
\begin{gathered}
\chi_{\mathrm{Rac}}^{s o(2,2)}(q, x) \chi_{\overline{\operatorname{Rac}}}^{s o(2,2)}(q, x)+\chi_{\mathrm{Rac}}^{s o(2,2)}(x, q) \chi_{\overline{\operatorname{Rac}}}^{s o(2,2)}(x, q) \\
=\chi_{(0,0)}^{s o(4)}(q, x)+\sum_{s=2}^{\infty} \chi_{(s-1, s-1)_{0}}^{s o(4)}(q, x),
\end{gathered}
$$

for the type-A model, and

$$
\begin{gathered}
\chi_{\mathrm{Di}}^{s o(2,2)}(q, x) \chi_{\overline{\mathrm{Di}}}^{s o(2,2)}(q, x)+\chi_{\mathrm{Di}}^{s o(2,2)}(x, q) \chi_{\overline{\mathrm{Di}}}^{s o(2,2)}(x, q) \\
=2 \chi_{(0,0)}^{s o(4)}(q, x)+\sum_{s=2}^{\infty} \chi_{(s-1, s-1)_{0}}^{s o(4)}(q, x),
\end{gathered}
$$

for the type-B model. Here, the so(4) module $(r, r)_{0}$ means the direct sum of the $(r, r)$ and $(r,-r)$ modules. Remark that the type-A model contains the trivial module $(0,0)$ once whereas the type-B model has it twice. They correspond to the Killing tensors of the Maxwell and $\mathrm{U}(1) \times \mathrm{U}(1)$ Chern-Simons theory, respectively.

In $\mathrm{AdS}_{3} / \mathrm{CFT}_{2}$, the higher-spin holography [75-77] involves more models than in higher dimensions: in fact, there is a one-parameter family of models which includes the type-A and type-B models as particular points in the parameter space. This parameter (corresponding to the 't Hooft coupling in the AdS/CFT context) is often denoted by $\lambda[70,73]$ (see also $[78,79]$ ) (or sometimes $\nu[80,81]$ ) and the chiral part of the underlying higher spin algebra is referred to as $h s[\lambda]$ and its asymptotic extension as $\mathcal{W}_{\infty}[\lambda]$ [82-85]. The former higher-spin algebra is the simple ${ }^{14}$ subalgebra of the Lie algebra (4.23):

$$
g l[\lambda]=\mathbb{R} \oplus h s[\lambda],
$$

\footnotetext{
${ }^{14}$ Except for $\lambda=N$ a positive integer, in which case it contains an infinite-dimensional ideal as described in (4.58).
} 
which is the endomorphism algebra of the modules $\mathcal{V}_{\frac{1 \pm \lambda}{2}}$ (the two modules of different signs have the same symmetry $g l[\lambda]$, as the latter depends on $\lambda$ only through its square $\left.\lambda^{2}\right)$. In the region $0 \leqslant \lambda<1$, both modules are unitary and irreducible. However, when $\lambda$ becomes a positive integer, say $N$, then $\mathcal{V}_{\frac{1+N}{2}}$ is a unitary irreducible submodule of the non-unitary reducible module $\mathcal{V}_{\frac{1-N}{2}}$. Moreover, the higher-spin algebra decomposes as the semidirect sum, ${ }^{15}$

$$
h s[N]=\operatorname{sl}(N) \Subset \mathcal{J}_{N},
$$

where $\mathcal{J}_{N}$ is an infinite-dimensional ideal of $h s[N]$ decomposing in irreducible modules of so $(2,1)$ as

$$
\mathcal{J}_{N}=\bigoplus_{j=N}^{\infty} \mathcal{D}_{j},
$$

while $s l(N)$ is a finite-dimensional higher-spin algebra which appears here as the symmetry of the irreducible module $\mathcal{D}_{\frac{N-1}{2}}=\mathcal{V}_{\frac{1-N}{2}} / \mathcal{V}_{\frac{1+N}{2}}$ and which decomposes as

$$
s l(N)=\bigoplus_{j=1}^{N-1} \mathcal{D}_{j} .
$$

This allows to shed some light on the comments below the twisted Flato-Fronsdal (4.50) for the spin- $s$ singleton in the chiral sector: the character (4.51) corresponds to the symmetry algebra $s l(2 s-1)$ of the Killing tensor $\mathcal{D}(s-1, s-1)=\mathcal{D}_{1-s} \otimes 1$ while the symmetry algebra of the $d=2$ spin- $s$ singleton $\mathcal{D}(s, s)=\mathcal{V}_{s} \otimes 1$ is isomorphic to the ideal $\mathcal{J}_{2 s-1}$.

Following the discussion at the end of section 4.1 on $g l[\lambda]$, one may say that the result (4.15) can be viewed as the twisted Flato-Fronsdal theorem relevant for the description of $h s[\lambda]$. In this sense, for a generic value of $\lambda$, one (or a combination) of the modules $\mathcal{V}_{\frac{1 \pm \lambda}{2}}$ ought to play the role of singleton. However, it does not seem possible to realize this picture in terms of a parity-invariant twisted Flato-Fronsdal theorem, except for the type-A and type-B models (cf (4.55)-(4.56)) which correspond respectively to $\lambda=1$ and $\lambda=0$ cases. Technically, it is because the character of the latter module does not have property similar to (4.52). In fact, it is known that the underlying CFT has a free field description only for $\lambda=0,1$.

\footnotetext{
${ }^{15}$ For a Lie algebra $\mathfrak{g}$ and the following semidirect sum of $\mathfrak{g}$-modules $U=V \notin W$ (where $W \subset U$ is the submodule and $V=U / W$ is the quotient module), the algebra $\mathcal{A}:=\mathcal{U}(\mathfrak{g}) / \operatorname{Ann}(U) \subset \operatorname{End}(U)$ preserves the submodule $W$ (i.e. $\mathcal{A} W \subset W$ ). Moreover, $\mathcal{A}$ decomposes as a semidirect sum,

$$
\mathcal{U}(\mathfrak{g}) / \operatorname{Ann}(U)=[\mathcal{U}(\mathfrak{g}) / \operatorname{Ann}(V)] \Subset \mathcal{I}
$$

where $\mathcal{I} \subset \mathcal{A}$ is the ideal spanned by the elements with image in $W$ (i.e. $\mathcal{I} U \subset W$ ). Notice that the latter property also holds in the simpler case when $U, V$ and $W$ are vector spaces (not necessarily with a $\mathfrak{g}$-module structure) in the sense that the subalgebra $\mathcal{A} \subset \operatorname{End}(U)$ of endomorphisms of $U$ preserving the subspace $W$ decomposes as a semidirect sum, $\mathcal{A}=\operatorname{End}(V) \oplus \mathcal{I}$, where $\mathcal{I}:=\mathcal{A} \cap \operatorname{Hom}(U, W)$.
} 


\section{$5 \quad$ Extensions and exceptions}

\subsection{Type $\mathbf{A}_{\ell}$}

Let us generalize the previous analysis to the type- $\mathrm{A}_{\ell}$ partially-massless higher-spin theory [9, 86-89]. This family of theories, parametrized by a positive integer $\ell$, involves not only infinitely many massless fields but also partially-massless fields with odd depth $t=1,3, \ldots, 2 \ell-1$. Its higher-spin algebra contains the corresponding Killing tensors, given by $s o(2+d)$ Young diagrams of the form $[9,90]$,

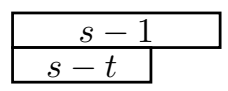

for odd $t=1,3, \ldots, 2 \ell-1$ and integer $s=t, t+1, \ldots$ The type- $\mathrm{A}_{\ell}$ partially-massless higherspin gravity in $d+1$ dimensions has been conjectured to be dual to the higher-derivative scalar CFT in $d$ dimensions with the polywave equation,

$$
\square^{\ell} \phi=0
$$

and the partially massless higher-spin algebra is the algebra of symmetries of the above equation. In other words, it is the endomorphism algebra of the solution space of (5.2), as showed in [91] for $\ell=2$ and generalized to arbitrary values of $\ell$ in [92] and [93]. This space carries an irreducible (but non-unitary for $\ell \geqslant 2$ ) representation,

$$
\operatorname{Rac}_{\ell}:=\mathcal{D}\left(\frac{d-2 \ell}{2}, \mathbf{0}\right)=\mathcal{V}\left(\frac{d-2 \ell}{2}, \mathbf{0}\right) / \mathcal{V}\left(\frac{d+2 \ell}{2}, \mathbf{0}\right),
$$

of the conformal algebra $s o(2, d)$. Its character reads

$$
\chi_{\operatorname{Rac}_{\ell}}^{s o(2, d)}(q, \boldsymbol{x})=q^{d / 2}\left(q^{-\ell}-q^{\ell}\right) \mathcal{P}_{d}(q, \boldsymbol{x}) .
$$

Using the property (3.7), this can be rewritten as

$$
\chi_{\operatorname{Rac}_{\ell}}^{s o(2, d)}(q, \boldsymbol{x})=\sum_{s=0}^{\infty} \sum_{k=0}^{\ell-1} q^{\frac{d-2 \ell}{2}+s+2 k} \chi_{s}^{s o(d)}(\boldsymbol{x})=\sum_{s=0}^{\infty} \sum_{t=1,3, \ldots}^{2 \ell-1} q^{\frac{d-2 \ell}{2}+s+t-1} \chi_{s}^{s o(d)}(\boldsymbol{x}) .
$$

The weight diagram of this representation can be immediately read off from the above formula, and is composed of $\ell$ lines ${ }^{16}$ similar to the one constituting the weight diagram of the original Dirac singleton (recovered in the case $\ell=1$ ) as depicted in figure 2 .

To derive, in the partially massless case, a twisted-Flato-Fronsdal theorem analogous to (3.19), we begin with the formula (3.8) applied to $(s-1, s-t)$ :

$$
\chi_{(s-1, s-t)}^{s o(2+d)}(\boldsymbol{x})=\sum_{k=0}^{r}\left(x_{k}^{1-s}+(-1)^{d} x_{k}^{s+d-1}\right) \chi_{s-t}^{s o(d)}\left(\boldsymbol{x}_{k}\right) \mathcal{P}_{d}\left(x_{k}, \boldsymbol{x}_{k}\right)
$$

\footnotetext{
${ }^{16}$ For this reason, Rac $\ell$ is sometimes referred to as "multipleton" [7], " $\ell$-lineton" [18] or "multilineton" $[10]$.
} 


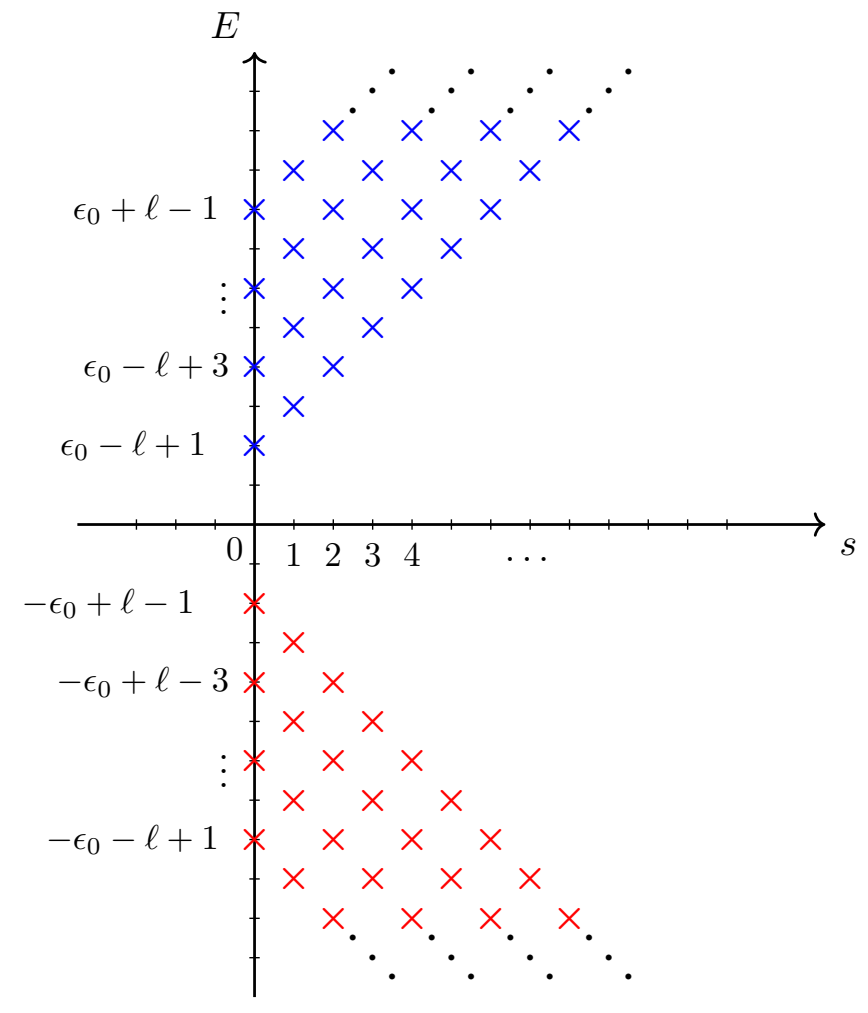

Figure 2. Weight diagram of the scalar, order $\ell$, singleton (blue crosses) and of the scalar, order $\ell$, anti-singleton (red crosses).

The summand of the above series satisfy

$$
\sum_{t=1,3, \ldots}^{2 \ell-1} \sum_{s=t}^{\infty}\left(x_{k}^{1-s}+(-1)^{d} x_{k}^{s+d-1}\right) \chi_{s-t}^{s o(d)}\left(\boldsymbol{x}_{k}\right)=\left(x_{k}^{-\ell}-x_{k}^{\ell}\right)\left(x_{k}^{\ell}-x_{k}^{-\ell}\right) \mathcal{P}_{d}\left(x_{k}^{-1}, \boldsymbol{x}_{k}\right),
$$

where both of the properties (3.6) and (3.7) are used for the derivation with the same subtleties related to convergence as in the previous sections. Collecting these results, we finally obtain the twisted-Flato-Fronsdal theorem for type- $\mathrm{A}_{\ell}$ partially massless higher-spin theory:

$$
\sum_{k=0}^{r} \chi_{\operatorname{Rac}_{\ell}}^{s o(2, d)}\left(x_{k}, \boldsymbol{x}_{k}\right) \chi_{\operatorname{Rac}_{\ell}}^{s o(2, d)}\left(x_{k}, \boldsymbol{x}_{k}\right)=\sum_{t=1,3, \ldots}^{2 \ell-1} \sum_{s=t}^{\infty} \chi_{(s-1, s-t)}^{s o(2+d)}\left(x_{0}, \boldsymbol{x}\right) .
$$

This result agrees with the collection of Killing tensors (5.1) and, thus, with the FlatoFronsdal theorem for type- $\mathrm{A}_{\ell}$ theory.

\section{$5.2 \quad$ Type $\mathrm{B}_{\ell}$}

Similarly to the type- $\mathrm{A}_{\ell}$ case, one can consider the partially-massless extension of the type$\mathrm{B}$ higher-spin algebra as the symmetry algebra of the higher-order spinor singleton that we will denote $\mathrm{Di}_{\ell}$, corresponding to the module:

$$
\mathcal{D}\left(\frac{d+1-2 \ell}{2}, \frac{\mathbf{1}}{\mathbf{2}}\right)=\frac{\mathcal{V}\left(\frac{d+1-2 \ell}{2}, \frac{\mathbf{1}}{\mathbf{2}}\right)}{\mathcal{D}\left(\frac{d-1+2 \ell}{2}, \frac{\mathbf{1}}{\mathbf{2}}\right)}
$$


with the character:

$$
\chi_{\mathrm{Di}_{\ell}}^{s o(2, d)}(q, \boldsymbol{x})=q^{\frac{d+1-2 \ell}{2}}\left(1-q^{2 \ell-1}\right) \chi_{\frac{1}{2}}^{s o(d)}(\boldsymbol{x}) \mathcal{P}_{d}(q, \boldsymbol{x}) .
$$

This corresponds to a spin- $\frac{1}{2}$ conformal field $\psi$, with conformal weight $\frac{d+1-2 \ell}{2}$ (non-unitary for $\ell \geqslant 2$ and corresponding to the Di for $\ell=1$ ) subject to the higher-order Dirac equation:

$$
\not \partial^{2 \ell-1} \psi=0 \text {. }
$$

The spectrum of possible bilinears in this fundamental field making up the currents of the type- $\mathrm{B}_{\ell}$ boundary theory was studied in $[10,94]$ and contains totally symmetric as well as "hook-shaped" partially-conserved currents of all spins (see [95-98] for more details on generic mixed-symmetry partially massless fields and [99] for the ones relevant here).

Notice that in odd $d+1$ bulk dimensions, these higher-order singletons can also be chiral as their unitary counter-part, i.e. one can consider the modules:

$$
\operatorname{Di}_{\ell \pm}:=\mathcal{D}\left(\frac{d+1-2 \ell}{2}, \frac{\mathbf{1}}{\mathbf{2}_{ \pm}}\right)=\frac{\mathcal{V}\left(\frac{d+1-2 \ell}{2}, \frac{\mathbf{1}}{\mathbf{2}}\right)}{\mathcal{D}\left(\frac{d-1+2 \ell}{2}, \frac{1}{2}_{\mp}\right)},
$$

whose character read:

$$
\chi_{\mathrm{Di}_{\ell \pm}}^{s o(2, d)}(q, \boldsymbol{x}):=q^{\frac{d+1-2 \ell}{2}}\left(\chi_{\frac{1}{2}_{ \pm}}^{s o(d)}(\boldsymbol{x})-q^{2 \ell-1} \chi_{\frac{1}{\mathbf{2}_{\mp}}}^{s o(d)}(\boldsymbol{x})\right) \mathcal{P}_{d}(q, \boldsymbol{x}) .
$$

We will however only consider the parity-invariant singleton, i.e. $\mathrm{Di}_{\ell}:=\mathrm{Di}_{\ell+} \oplus \mathrm{Di}_{\ell_{-}}$, so as to be able to treat both the odd and even dimensional cases on an equal footing. The only subtlety to keep in mind when reading the decomposition hereafter is that for $d=2 r$, all diagrams of maximal height (namely $r+1$ for $s o(2+d)$ ) come with both chiralities, whereas all other diagrams come with a multiplicity 2 .

Using the fact that the character of this higher-order singleton can be expanded as

$$
\left.\chi_{\mathrm{Di}_{\ell}}^{s o(2, d)}(q, \boldsymbol{x})=\sum_{k=0}^{2(\ell-1)} \sum_{s=0}^{\infty} q^{\frac{d+1-2 \ell}{2}+s+k} \chi_{\left(s+\frac{1}{2}, \frac{1}{2}\right.}^{s o(d)}\right)(\boldsymbol{x}),
$$

and after a calculation similar to that of the previous section, one can show that the tensor product of the $\mathrm{Di}_{\ell}$ singleton and its anti-singleton can be decomposed as

$$
\begin{aligned}
\sum_{k=0}^{r} \chi_{\mathrm{Di}_{\ell}}^{s o(2, d)}\left(x_{k}, \boldsymbol{x}_{k}\right) \times \chi_{\overline{\mathrm{Di}}_{\ell}}^{s o(2, d)}\left(x_{k}, \boldsymbol{x}_{k}\right) \\
=\sum_{t=1}^{2 \ell-1}\left[\chi_{t-1}^{s o(2+d)}\left(x_{0}, \boldsymbol{x}\right)+\sum_{s=t+1}^{\infty} \sum_{m=0}^{r-1} \chi_{\left(s-1, s-t, 1^{m}\right)}^{s o(2+d)}\left(x_{0}, \boldsymbol{x}\right)\right] \\
\quad+\sum_{t=1}^{2 \ell-2} \sum_{s=t+1}^{\infty} \sum_{m=0}^{r-1} \chi_{\left(s-1, s-t, 1^{m}\right)}^{s o(2+d)}\left(x_{0}, \boldsymbol{x}\right) .
\end{aligned}
$$

Notice that the last triple sum in the above decomposition is absent for $\ell=1$, in which case the second line reproduces the spectrum of the type-B higher-spin algebra discussed in the previous subsection (whereas the last line identically vanishes). 
Notice also that the spectrum of the higher-spin theories so far considered — which is obtained by decomposing the tensor product of the relevant singletons - is closely related to the $s o(2+d)$ decomposition of the underlying higher-spin algebra: the later is composed of the Killing tensors of the all the (partially) massless fields appearing in the former. We were able to recover these decompositions from the tensor product of the relevant singleton and its anti-singletons for the type- $\mathrm{A}_{\ell}$ (with $\ell \geqslant 1$ ) and type-B higherspin algebras, however it seems to fail in the case of the type- $\mathrm{B}_{\ell}$ algebra (with $\ell>1$ ). Indeed, the spectrum of partially-massless fields appearing in the tensor product of two $\mathrm{Di}_{\ell}$ singletons reads $[10]$

$$
\begin{aligned}
\mathrm{Di}_{\ell}^{\otimes 2} \supset & \bigoplus_{t=1}^{2 \ell-1}\left[\mathcal{D}(d-1, t) \oplus \bigoplus_{s=t+1}^{\infty} \bigoplus_{m=0}^{r-1} \mathcal{D}\left(s+d-t-1, s, 1^{m}\right)\right] \\
& +\bigoplus_{t=1}^{2 \ell-2}\left[\mathcal{D}(d-1, t) \oplus \bigoplus_{s=t+1}^{\infty} \bigoplus_{m=0}^{r-1} \mathcal{D}\left(s+d-t-1, s, 1^{m}\right)\right]
\end{aligned}
$$

and in particular contains two infinite towers of totally symmetric partially massless fields, of all depths $t$ ranging from 1 to (respectively) either $2 \ell-1$ or $2 \ell-2$, and of all integer spins $s \geqslant t$. It therefore seems that the set of Killing tensors corresponding to totally symmetric partially-massless fields of spin $s=t$ are missing for $t=1,2, \ldots, 2 \ell-2$, i.e. one would expect that the sum

$$
\sum_{t=1}^{2 \ell-2} \chi_{t-1}^{s o(2+d)}\left(x_{0}, \boldsymbol{x}\right)
$$

should be added to (5.15) in order make up the spectrum of the type- $\mathrm{B}_{\ell}$ higher-spin algebras. This is the first discrepancy that we find in our proposal.

\subsection{Type $\mathrm{AB}_{\ell}$}

The type- $\mathrm{AB}_{\ell}$ theory includes the cross terms of the Rac and Di $\ell$-linetons, on top of the contents of the type- $\mathrm{A}_{\ell}$ and type- $\mathrm{B}_{\ell}$ theories. Since we have examined the latter cases in the previous section, here we focus on the cross terms.

Using (3.33), the product of the character of a Rac singleton with that of the $\overline{\mathrm{Di}}$ anti-singleton can be written as

$$
\left.\chi_{\operatorname{Rac}_{\ell}}^{s o(2, d)}(q, \boldsymbol{x}) \times \chi_{\overline{\mathrm{Di}} \ell_{\ell}}^{s o(2, d)}(q, \boldsymbol{x})=q^{-\frac{1}{2}}\left(1-q^{2 \ell}\right) \mathcal{P}_{d}(q, \boldsymbol{x}) \sum_{s=0}^{\infty} \sum_{k=0}^{2(\ell-1)} q^{-s-k} \chi_{\left(s+\frac{1}{2}, \frac{1}{2}\right.}^{s-1}\right)(\boldsymbol{x}),
$$

whereas for $\overline{\operatorname{Rac}}$ with Di as

$$
\left.\chi_{\overline{\operatorname{Rac}}_{\ell}}^{s o(2, d)}(q, \boldsymbol{x}) \times \chi_{\mathrm{Di}_{\ell}}^{s o(2, d)}(q, \boldsymbol{x})=q^{\frac{1}{2}}\left(1-q^{-2 \ell}\right) \mathcal{P}_{d}\left(q^{-1}, \boldsymbol{x}\right) \sum_{s=0}^{\infty} \sum_{k=0}^{2(\ell-1)} q^{s+k} \chi_{\left(s+\frac{1}{2}, \frac{1}{2}\right.}^{s-1}\right)(\boldsymbol{x}) .
$$


Their sum can be simplified to

$$
\begin{aligned}
\chi_{\mathrm{Rac}_{\ell}}^{s o(2, d)}(q, \boldsymbol{x}) \times \chi_{\overline{\mathrm{Di}}_{\ell}}^{s o(2, d)}(q, \boldsymbol{x})+\chi_{\overline{\mathrm{Rac}}_{\ell}}^{s o(2, d)}(q, \boldsymbol{x}) \times \chi_{\mathrm{Di}_{\ell}}^{s o(2, d)}(q, \boldsymbol{x}) \\
=\sum_{t=1}^{2 \ell-1} \sum_{s=t}^{\infty}\left[\left(q^{-s+\frac{1}{2}}+(-1)^{d} q^{s+d-\frac{1}{2}}\right) \chi_{\left(s-t+\frac{1}{2}, \frac{1}{2}\right.}^{s-1}\right)(\boldsymbol{x}) \mathcal{P}_{d}(q, \boldsymbol{x}) \\
\left.\left.-\left(q^{-s+\frac{1}{2}+t}+(-1)^{d} q^{s+d-\frac{1}{2}-t}\right) \chi_{\left(s+\frac{1}{2}, \frac{1}{2}\right.}^{s o(d)}\right)(\boldsymbol{x}) \mathcal{P}_{d}(q, \boldsymbol{x})\right] .
\end{aligned}
$$

Symmetrizing the $r+1$ variables of the above expression and using (3.8), we end up with the following sum of $s o(2+d)$ characters

$$
\begin{array}{r}
\sum_{k=0}^{r}\left(\chi_{\mathrm{Rac}_{\ell}}^{s o(2, d)}\left(x_{k}, \boldsymbol{x}_{k}\right) \times \chi_{\overline{\mathrm{Di}}_{\ell}}^{s o(2, d)}\left(x_{k}, \boldsymbol{x}_{k}\right)+\chi_{\overline{\mathrm{Rac}}_{\ell}}^{s o(2, d)}\left(x_{k}, \boldsymbol{x}_{k}\right) \times \chi_{\mathrm{Di}_{\ell}}^{s o(2, d)}\left(x_{k}, \boldsymbol{x}_{k}\right)\right) \\
\left.\left.=\sum_{t=1}^{2 \ell-1} \sum_{s=t}^{\infty} \chi_{\left(s-\frac{1}{2}, s-t+\frac{1}{2}, \frac{1}{2}\right.}^{s-1}\right)\left(x_{0}, \boldsymbol{x}\right)-\sum_{t=1}^{2 \ell-1} \sum_{s=0}^{\infty} \chi_{\left(s-t-\frac{1}{2}, s+\frac{1}{2}, \frac{1}{2}\right.}^{s-1}\right)\left(x_{0}, \boldsymbol{x}\right) .
\end{array}
$$

Using the symmetry property (3.10), the characters appearing in the second sum can be expressed as characters of bona fide $s o(2+d)$ Young diagrams, given that

$$
\chi_{\left(s-t-\frac{1}{2}, s+\frac{1}{2}, \frac{1}{2}\right.}^{s o(d+1)}=-\chi_{\left(s-\frac{1}{2}, s-t+\frac{1}{2}^{2}, \frac{1}{2}^{r-1}\right)}^{s o(d+2)} .
$$

Moreover, due to the same property, the sum of the characters for which $s<t$ identically vanish. Hence, we end up with

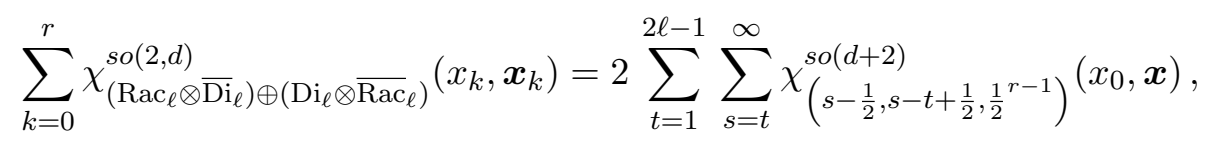

which is consistent with the corresponding Flato-Fronsdal theorem, giving the decomposition of the tensor product $\operatorname{Rac}_{\ell} \otimes \operatorname{Di}_{\ell}[10]$.

\section{$5.4 \quad$ Type J}

In even boundary dimensions $d=2 r$, we have infinitely many singleton representations $[100,101]$ corresponding to

$$
\mathcal{S}_{j \pm}=\mathcal{D}\left(j+r-1, \mathbf{j}_{ \pm}\right) \quad \text { with } \quad \mathbf{j}_{ \pm}:=(j, \ldots, j, \pm j) .
$$

Their characters can be written in terms of the ones of the lowest-weight module $\mathcal{V}(\Delta, \ell)$ as

$$
\begin{aligned}
\chi_{\mathcal{S}_{j \pm}}(q, \boldsymbol{x}) & =\sum_{m=0}^{r}(-1)^{m} \chi_{\left.\mathcal{V}\left(j+r-1+m, j^{r-m},(j-1)_{ \pm}^{m}\right)\right)}(q, \boldsymbol{x}) \\
& =q^{j+r-1} \mathcal{P}_{2 r}(q, \boldsymbol{x}) \sum_{m=0}^{r}(-q)^{m} \chi_{\left(j^{r-m},(j-1)_{ \pm}^{m}\right)}^{s o(2 r)}(\boldsymbol{x})
\end{aligned}
$$


Similarly to the other singletons that we have seen above, these higher-spin singletons for even $d$ satisfy the property,

$$
\chi_{\mathcal{S}_{j \pm}}(q, \boldsymbol{x})=\sum_{s=0}^{\infty} q^{j+r+s-1} \chi_{\left(s+j, j_{ \pm}^{r-1}\right)}^{s o(d)}(\boldsymbol{x}),
$$

from which the term "singleton" originated [102] (cf the historical comment in [103]). The anti-singleton is the highest-weight counterpart of the singleton, and its character is

$$
\chi_{\mathcal{S}_{j \pm}}(q, \boldsymbol{x})=\chi_{\mathcal{S}_{j \pm}}\left(q^{-1}, \boldsymbol{x}^{-1}\right)=\left\{\begin{array}{ll}
\chi_{\mathcal{S}_{j \pm}}\left(q^{-1}, \boldsymbol{x}\right) & {[\text { even } r]} \\
\chi_{\mathcal{S}_{j \mp}}\left(q^{-1}, \boldsymbol{x}\right) & {[\text { odd } r]}
\end{array} .\right.
$$

Remark here that the singleton character $\chi_{\mathcal{S}_{j \pm}}(q, \boldsymbol{x})$ does not have a simple property under $q \rightarrow q^{-1}$, even in the parity-invariant case, contrarily to the previously treated $\operatorname{Rac}_{\ell}$ and $\mathrm{Di}_{\ell}$ singletons. We now consider the product of these two characters:

$$
\chi_{\mathcal{S}_{j \sigma}}(q, \boldsymbol{x}) \chi_{\overline{\mathcal{S}_{j \tau}}}(q, \boldsymbol{x})=\mathcal{P}_{2 r}(q, \boldsymbol{x}) \sum_{s=0}^{\infty} \sum_{m=0}^{r} q^{m-s}(-1)^{m} \chi_{\left(j^{r-m},(j-1)_{\sigma}^{m}\right)}^{s o(2 r)}(\boldsymbol{x}) \chi_{\left(s+j, j_{\tau}-1\right)}^{s o(2 r)}(\boldsymbol{x}),
$$

where $\sigma$ and $\tau$ stands for the signs \pm . To proceed, we need to decompose the representation,

$$
\left(j^{r-m},(j-1)_{\sigma}^{m}\right) \otimes\left(s+j, j_{\tau}^{r-1}\right),
$$

into $s o(2 r)$ irreps. Unfortunately this task is quite cumbersome for generic value of $j, s, m, r$, hence we focus on the particular case $d=4$ in the rest of this section.

Type-J in five dimensions. For $d=4$, the Flato-Fronsdal theorem has been derived in [11] as

$$
\begin{aligned}
& \chi_{\mathcal{S}_{j \pm}} \times \chi_{\mathcal{S}_{j \pm}}=\sum_{k=0}^{2 j} \chi_{\mathcal{D}(2 j+2, k, \pm k)}^{s o(2,4)}(q, \boldsymbol{x})+\sum_{s=2 j+1}^{\infty} \chi_{\mathcal{D}(s+2, s, \pm 2 j)}^{s o(2,4)}(q, \boldsymbol{x}), \\
& \chi_{\mathcal{S}_{j \pm}} \times \chi_{\mathcal{S}_{j \mp}}=\sum_{s=2 j}^{\infty} \chi_{\mathcal{D}(s+2, s)}^{s o(2,4)}(q, \boldsymbol{x}) .
\end{aligned}
$$

Let us consider the corresponding twisted Flato-Fronsdal theorem. The tensor product (5.29) can be computed more easily thanks to the low dimensional isomorphism $s o(4) \cong$ $s o(3) \oplus s o(3)$. Consequently, an $s o(4)$ irrep with highest weight $\left(\ell_{1}, \ell_{2}\right)$ is equivalent to the direct sum of two $s o(3)$ irreps with highest-weights $j_{ \pm}:=\frac{\ell_{1} \pm \ell_{2}}{2}$, and the tensor product of two arbitrary representations of $s o(4)$ reduces to the tensor products of their two respective so(3) factors, for which we can use the well-known Clebsch-Gordan decomposition (4.7). Applying it to the formula (5.28) with $\sigma=+$ and $\tau=-$, we obtain

$$
\begin{aligned}
\chi_{\mathcal{S}_{j+}}(q, \boldsymbol{x}) \chi_{\overline{\mathcal{S}_{j-}}}(q, \boldsymbol{x})= & {\left[\sum_{s=0}^{2 j-2} q^{2-s} \chi_{(2 j-1,-s-1)}^{s o(4)}(\boldsymbol{x})-\sum_{s=0}^{2 j-1} q^{1-s} \chi_{(2 j-1,-s)}^{s o(4)}(\boldsymbol{x})\right.} \\
& \left.+\sum_{s=2 j}^{\infty} q^{-s} \chi_{(s,-2 j)}^{s o(4)}(\boldsymbol{x})-\sum_{s=2 j}^{\infty} q^{2-s} \chi_{(s,-2 j)}^{s o(4)}(\boldsymbol{x})\right] \mathcal{P}_{4}(q, \boldsymbol{x}) .
\end{aligned}
$$


The same for $\sigma=-, \tau=+$ with variable $q^{-1}$ gives

$$
\begin{aligned}
\chi_{\mathcal{S}_{j+}}(q, \boldsymbol{x}) \chi_{\overline{\mathcal{S}_{j-}}}(q, \boldsymbol{x})= & {\left[\sum_{s=0}^{2 j-2} q^{s+2} \chi_{(2 j-1, s+1)}^{s o(4)}(\boldsymbol{x})-\sum_{s=0}^{2 j-1} q^{s+3} \chi_{(2 j-1, s)}^{s o(4)}(\boldsymbol{x})\right.} \\
& \left.+\sum_{s=2 j}^{\infty} q^{s+4} \chi_{(s, 2 j)}^{s o(4)}(\boldsymbol{x})-\sum_{s=2 j}^{\infty} q^{s+2} \chi_{(s, 2 j)}^{s o(4)}(\boldsymbol{x})\right] \mathcal{P}_{4}(q, \boldsymbol{x}) .
\end{aligned}
$$

Symmetrizing the half sum of (5.32) and (5.33), we find

$$
\sum_{k=0}^{2} \chi_{\mathcal{S}_{j \pm}}\left(x_{k}, \boldsymbol{x}_{k}\right) \chi_{\overline{\mathcal{S}_{j \mp}}}\left(x_{k}, \boldsymbol{x}_{k}\right)=\sum_{s=2 j+1}^{\infty} \chi_{(s-1, s-1, \pm 2 j)}^{s o(6)}\left(x_{0}, \boldsymbol{x}\right)+\sum_{s=0}^{2 j-2} \chi_{(2 j-2, s, \pm s)}^{s o(6)}\left(x_{0}, \boldsymbol{x}\right),
$$

where we have included also the product $\mathcal{S}_{j-}$ and $\overline{\mathcal{S}_{j+}}$ using the relation (5.27). Let us comment about the two series in the right-hand-side of the equality. Since the $s o(6)$ irrep $(s-1, s-1,2 j)$ is carried by the Killing tensor of the spin- $(s, 2 j)$ gauge field, the first infinite series matches well the content of gauge fields in the Flato-Fronsdal theorem (5.31). However, this spectrum does not include the gauge fields corresponding to the second finite series. In fact, the second series can be written as a perfect square:

$$
\sum_{s=0}^{2 j-2} \chi_{(2 j-2, s, \pm s)}^{s o(6)}=\left(\chi_{(j-1, j-1, \pm(j-1))}^{s o(6)}\right)^{2}=\chi_{(j-1, j-1, \pm(j-1))}^{s o(6)} \chi_{(j-1, j-1, \mp(j-1))}^{s o(6)}
$$

where the $s o(6)$ Young diagrams $(j-1, j-1, \pm(j-1))$ are the Killing tensor of the spin- $\pm j$ singleton. This factorization is analogous to the $d=2$ case (4.51). It is interesting to note the identity,

$$
\chi_{(j-1, j-1, \pm(j-1))}^{s o(6)}(q, \boldsymbol{x})=\chi_{\mathcal{S}_{j \pm}}(q, \boldsymbol{x})+\chi_{\overline{\mathcal{S}_{j \mp}}}(q, \boldsymbol{x}),
$$

which is again somewhat analogous to the two-dimensional one (4.5).

Similarly, the product of the character of a spin- $j$ singleton of positive/negative chirality with the character of its own anti-singleton can be decomposed into two different forms: firstly,

$$
\begin{aligned}
& \chi_{\mathcal{S}_{j \pm}}(q, \boldsymbol{x}) \chi_{\overline{\mathcal{S}_{j \pm}}}(q, \boldsymbol{x})= \\
& \quad=\left[\sum_{s=0}^{\infty} q^{-s} \chi_{s}^{s o(4)}(\boldsymbol{x})-\sum_{s=0}^{\infty} q^{1-s} \chi_{(s+1)}^{s o(4)}(\boldsymbol{x})+\sum_{s=1}^{2 j-1} q^{2} \chi_{(s, \pm s)}^{s o(4)}(\boldsymbol{x})\right] \mathcal{P}_{4}(q, \boldsymbol{x}),
\end{aligned}
$$

and secondly,

$$
\begin{aligned}
\chi_{\mathcal{S}_{j \pm}} & (q, \boldsymbol{x}) \chi_{\overline{\mathcal{S}_{j \pm}}}(q, \boldsymbol{x})=\chi_{\mathcal{S}_{j \pm}}\left(q^{-1}, \boldsymbol{x}^{-1}\right) \chi_{\overline{\mathcal{S}_{j \pm}}}\left(q^{-1}, \boldsymbol{x}^{-1}\right) \\
\quad= & {\left[\sum_{s=0}^{\infty} q^{s+4} \chi_{s}^{s o(4)}(\boldsymbol{x})-\sum_{s=0}^{\infty} q^{s+3} \chi_{(s+1)}^{s o(4)}(\boldsymbol{x})+\sum_{s=1}^{2 j-1} q^{2} \chi_{(s, \pm s)}^{s o(4)}(\boldsymbol{x})\right] \mathcal{P}_{4}(q, \boldsymbol{x}) . }
\end{aligned}
$$


After symmetrization, the half sum of these two decompositions yields

$$
\begin{aligned}
\sum_{k=0}^{2} \chi_{\mathcal{S}_{j \pm}}\left(x_{k}, \boldsymbol{x}_{k}\right) \chi_{\overline{\mathcal{S}_{j \pm}}}\left(x_{k}, \boldsymbol{x}_{k}\right)= & \sum_{s=1}^{2 j-1} \chi_{(s-1, s-1)}^{s o(6)}\left(x_{0}, \boldsymbol{x}\right)+\sum_{s=2 j}^{\infty} \chi_{(s-1, s-1)}^{s o(6)}\left(x_{0}, \boldsymbol{x}\right) \\
& +\sum_{s=1}^{2 j-1} \sum_{k=0}^{2} \chi_{(s, \pm s)}^{s o(4)}\left(\boldsymbol{x}_{k}\right) x_{k}^{2} \mathcal{P}_{4}\left(x_{k}, \boldsymbol{x}_{k}\right)
\end{aligned}
$$

In the first line, the two-row Young diagrams $(s-1, s-1)$ for $s \geqslant 2 j$ correspond to the Killing tensors of the totally symmetric massless fields that appear in the tensor product of two spin- $j$ singletons of opposite chirality. The additional two-row diagrams for $1 \leqslant s \leqslant 2 j-1$ can be interpreted as the result of the tensor product of the Killing tensors of the spin- $\pm j$ singleton and its dual:

$$
\chi_{(j-1, j-1, \pm(j-1))}^{s o(6)}\left(x_{0}, \boldsymbol{x}\right) \chi_{\frac{s o(6)}{(j-1, j-1, \pm(j-1))}}\left(x_{0}, \boldsymbol{x}\right)=\sum_{k=0}^{2 j-2} \chi_{(k, k)}^{s o(6)}\left(x_{0}, \boldsymbol{x}\right) .
$$

If the second line of (5.39) were absent, the above result matches well the symmetry of the $d=4$ spin- $j$ chiral singleton: the modules $(s-1, s-1)$ with $s \geqslant 2 j$ correspond to the ideal part of the symmetry, while the rest with $1 \leqslant s \leqslant 2 j-1$ correspond to the quotient part $[40,104,105]$. Since the character is not sensitive to the indecomposability, it is natural that we get both the ideal and quotient algebras here. However, the second line of (5.39) does include additional terms. We do not have clear interpretation of these terms.

Let us conclude this section with the twisted Flato-Fronsdal of the parity-invariant spin- $j$ singleton, having character $\chi_{\mathcal{S}_{j}}=\chi_{\mathcal{S}_{j_{+}}}+\chi_{\mathcal{S}_{j_{-}}}$. By collecting the previous results (5.34) and (5.39), we obtain

$$
\begin{aligned}
& \sum_{k=0}^{2} \chi_{\mathcal{S}_{j}}\left(x_{k}, \boldsymbol{x}_{k}\right) \chi_{\overline{\mathcal{S}_{j}}}\left(x_{k}, \boldsymbol{x}_{k}\right) \\
& =2 \sum_{s=2 j}^{\infty} \chi_{(s-1, s-1)}^{s o(6)}\left(x_{0}, \boldsymbol{x}\right)+\sum_{s=2 j+1}^{\infty} \chi_{(s-1, s-1,2 j)_{0}}^{s o(6)}\left(x_{0}, \boldsymbol{x}\right) \\
& \quad+\left(\chi_{(j-1, j-1, j-1)_{0}}^{s o(6)}\left(x_{0}, \boldsymbol{x}\right)\right)^{2}+\sum_{s=1}^{2 j-1} \chi_{(s-1, s-1)}^{s o(6)}\left(x_{0}, \boldsymbol{x}\right)
\end{aligned}
$$

Here the subscript 0 of the so(6) modules signals that they are the direct sum of the two chiral representations. Remark that the terms in the second line and the first term in the third line correspond to the symmetry algebra of the parity-invariant spin- $j$ singleton. The last term of the third line is from the additional terms in (5.39). By adding up two contributions from $\chi_{\mathcal{S}_{j+}} \chi_{\overline{\mathcal{S}_{j+}}}$ and $\chi_{\mathcal{S}_{j-}} \chi_{\overline{\mathcal{S}_{j-}}}$, such terms form the so(6) character written above. These modules are in fact a part of the generators of the quotient higher-spin algebra. However, the quotient algebra was already taken into account by the first term in the third line. Therefore, these modules are additional and do not match with the symmetry algebra of the spin- $j$ singleton. 


\section{Discussion}

In this paper, we have explored the relation between the $s o(2, d)$ characters of the singletons and the adjoint module of higher-spin algebras. Starting from the idea that the higher-spin algebra is the endomorphism algebra of the singleton module, we attempted to derive the character for the adjoint module as a product of the singleton character and its dual. We first noticed that a simple product of the characters cannot reproduce the adjoint module one because the latter is symmetric under the exchange of its arguments while the former lacks this symmetry. This lead to our symmetrization prescription of the character product.

In section 2, we used the oscillator realization of the singleton and higher-spin algebra in four dimensions to relate the extra term (arising from the symmetrization prescription) in the character to an extra piece (with respect to the naive tensor product) in the twisted Flato-Fronsdal theorem (2.48). In section 3, we showed that the symmetrization prescription correctly reproduces the adjoint module character for the type-A and type- $\mathrm{B}$ models in any dimension. This is based on several interesting identities of the $s o(2, d)$ and $s o(2+d)$ characters, which have their root in the Weyl character formula. In section 5 , the symmetrization prescription was shown to work for the higher-order singleton case of type- $\mathrm{A}_{\ell}$. However, in the type- $\mathrm{B}_{\ell}$ theory, we found that the symmetrized product misses a few Killing tensor modules (5.17). Moreover, for the higher-spin singletons, aka the type$\mathrm{J}$ model, the symmetrized product contains more Killing tensor modules than necessary, cf (5.41). In both counterexamples, the mismatch is by a finite number of modules.

The symmetrization prescription of the character arguments can be viewed as an action of certain Weyl group elements. Remember that the Weyl group of a semisimple Lie algebra maps a Cartan subalgebra to itself. Since the variables that we symmetrize for the twisted Flato-Fronsdal theorem are associated to the Cartan generators, the symmetrization prescription can be induced by the action of the Weyl group quotiented by its normalizer subgroup of the singleton and anti-singleton tensor product. Referring to such quotient group as $\mathcal{W}^{\prime}$, we can restate our prescription in terms of the modules themselves as

$$
\operatorname{Adj}=\bigoplus_{w \in \mathcal{W}^{\prime}} w(\mathrm{Sng}) \otimes w(\overline{\mathrm{Sng}}) .
$$

In the case $d=3$, this prescription reproduces the twisted Flato-Fronsdal theorem (2.51). In the singleton module - and in all other lowest-weight modules - the energy generator $E$ plays a distinguished role with respect to the other Cartan generators, which belong to the rotation subalgebra $s o(d)$. The action of the aforementioned Weyl group elements symmetrize $E$ with those other Cartan generators. Here, it is interesting to note that such an action will map the singleton module to a non-unitary module.

In contrast, the Killing tensors are already symmetric under this action, hence should not be "over-symmerized". Maybe the application of the symmetrization prescription to the type-J singleton mistreats this subtle point and is the reason for the appearance of the anomalous finite-dimensional module (the last term in (5.41)).

To recapitulate, the heuristic prescription of symmetrization of the character arguments works surprisingly well for type-A and type-B models, as well as type- $\mathrm{A}_{\ell}$, but we 
also found some finite-dimensional discrepancy when the underlying singleton module is a non-standard one and has more complicated structure. This clearly suggests that our prescription should have a more refined meaning and asks for further investigations. One direction worth exploring would be to analyze the type-A (or beyond: type-B, type-C, etc) higher-spin algebra as the quotient of the universal enveloping algebra of $s o(2, d)$ by the Joseph ideal (or, respectively, more complicated primitive ideals). By comparing the basis of such quotient space with the lowest-weight module structure, we should be able to identify the origin of the symmetrization and understand the finite-dimensional mismatch in the cases beyond type-B. However, such a work is beyond the scope of the current investigation and will be explored elsewhere.

\section{Acknowledgments}

T.B. is grateful to N. Boulanger and C. Iazeolla for useful discussions on group theoretical issues discussed in the present work, as well as to D. Ponomarev for exchanges on anti-singletons. We are also grateful to an anonymous referee for insightful comments and suggestions. The research of T.B. and E.J. was supported by the National Research Foundation (Korea) through the grant 2014R1A6A3A04056670. The research of X.B. was supported by the Russian Science Foundation grant 14-42-00047 in association with the Lebedev Physical Institute.

\section{A Generalized Verma modules}

Recall that the usual commutation relations of $s o(2, d)$ read

$$
\left[M_{A B}, M_{C D}\right]=i\left(\eta_{B C} M_{A D}-\eta_{A C} M_{B D}-\eta_{B D} M_{A C}+\eta_{A D} M_{B C}\right),
$$

where $A, B, \ldots,=0,0^{\prime}, 1, \ldots, d$, the generators are antisymmetric and Hermitian, $M_{A B}=$ $M_{A B}^{\dagger}=-M_{B A}$, and $\eta:=\operatorname{diag}(-1,-1,1, \ldots, 1)$. We define

$$
E:=M_{0^{\prime} 0}, \quad L_{a}^{+}:=M_{0 a}-i M_{0^{\prime} a}, \quad L_{a}^{-}:=M_{0 a}+i M_{0^{\prime} a},
$$

where $a, b=1, \ldots, d$. In terms of these generators, the above commutation relations (A.1) can be rewritten:

$$
\left[E, L_{a}^{ \pm}\right]= \pm L_{a}^{ \pm}, \quad\left[L_{a}^{-}, L_{b}^{+}\right]=2\left(i M_{a b}+\delta_{a b} E\right), \quad\left[M_{a b}, L_{c}^{ \pm}\right]=2 i \delta_{c[b} L_{a]}^{ \pm},
$$

together with the $s o(d)$ subalgebra commutation relations

$$
\left[M_{a b}, M_{c d}\right]=i\left(\delta_{b c} M_{a d}-\delta_{a c} M_{b d}-\delta_{b d} M_{a c}+\delta_{a d} M_{b c}\right) .
$$

The $s o(2, d)$ generalized Verma modules $\mathcal{V}(\Delta, \ell)$ considered in this work are the modules induced from finite-dimensional modules $\mathbb{V}_{[\Delta ; \ell]}$ of the parabolic subalgebra spanned by $E, M_{a b}$ and $L_{c}^{-}$as follows: 
- The finite-dimensional module $\mathbb{V}_{[\Delta ; \ell]}$ carries a representation of $s o(d)$ with highest weight $\ell=\left(\ell_{1}, \ldots, \ell_{r}\right)$ where $r=\left[\frac{d}{2}\right]$ is the rank of $s o(d)$ and a (one-dimensional) representation of the $s o(2)$ algebra spanned by $E$ characterized by the weight $\Delta$. In other words, every element of $\mathbb{V}_{[\Delta ; \ell]}$ is an eigenvector of $E$ with eigenvalue $\Delta$. Finally, generators $L_{a}^{-}$are represented trivially on $\mathbb{V}_{[\Delta ; \ell]}$, i.e. the module is annihilated by the action of these lowering operators.

- The generalized Verma modules $\mathcal{V}(\Delta, \ell)$ is freely generated by the action of the raising operators $L_{a}^{+}$, i.e. it is composed of elements of the form:

$$
L_{a_{1}}^{+} \ldots L_{a_{n}}^{+} \mathbb{V}_{[\Delta ; \ell]} \in \mathcal{V}(\Delta, \ell) \quad \text { for } n \in \mathbb{N}
$$

\section{B Weyl character formula}

We make use of the notations introduced in section 3 and we will give a derivation of formula (3.8) from the Weyl character formula. This formula expresses the character $\chi_{\lambda}$ of a finite-dimensional, irreducible representation of a complex semi-simple Lie algebra $\mathfrak{g}$ as

$$
\chi_{\lambda}=\frac{\sum_{w \in \mathcal{W}} \varepsilon(w) e^{w(\lambda+\rho)-\rho}}{\prod_{\alpha \in \Phi_{+}}\left(1-e^{-\alpha}\right)},
$$

where $\lambda$ is the highest-weight labeling the representation, $\mathcal{W}$ is the Weyl group of $\mathfrak{g}, \varepsilon(w)$ is the signature of a Weyl group element and $\rho:=\frac{1}{2} \sum_{\alpha \in \Phi_{+}} \alpha$ is the Weyl vector of $\mathfrak{g}$ defined as the half-sum of all the positive roots (represented by the set $\Phi_{+}$) of $\mathfrak{g}$. We are interested in $\mathfrak{g}=s o(2+d)$, for which the Weyl group is $\mathcal{W} \cong \mathcal{S}_{r+1} \ltimes\left(\mathbb{Z}_{2}\right)^{r+1}$ for $d=2 r+1$ and $\mathcal{W} \cong \mathcal{S}_{r+1} \ltimes\left(\mathbb{Z}_{2}\right)^{r}$ for $d=2 r$. In other words, the Weyl group acts as the semi-direct product of the permutation group of $r+1$ elements with a group of "sign flips" on the $r+1$ components of an $s o(2+d)$ weight. More concretely, an element $w \in \mathcal{W}$ of the Weyl group first flips the sign of a number of components of the $s o(2+d)$ weight (an arbitrary number of components for $d=2 r+1$ and only an even number for $d=2 r$ ) and then permutes these $r+1$ components.

Formally, Lie algebra characters are maps from the weight space of the algebra (which is isomorphic to the dual of the Cartan subalgebra $\mathfrak{h} \subset \mathfrak{g}$ ) to the field of complex numbers:

$$
\chi_{\lambda}: \mathfrak{h}^{*} \rightarrow \mathbb{C}
$$

The evaluation of expression (B.1) on an arbitrary weight $\mu$ is defined through

$$
e^{\lambda}(\mu):=e^{(\lambda, \mu)},
$$

where $($,$) denotes the Killing form of \mathfrak{g}$, which is simply the Euclidean inner product on the weight space, $\mathfrak{h}^{*} \cong \mathbb{R}^{r+1}$ for $s o(2+d)$. As a consequence, the formula (B.1), when evaluated on a weight $\mu$, reads

$$
\chi_{\lambda}(\mu)=\frac{\sum_{w \in \mathcal{W}} \varepsilon(w) e^{(w(\lambda+\rho)-\rho, \mu)}}{\prod_{\alpha \in \Phi_{+}}\left(1-e^{-(\alpha, \mu)}\right)} .
$$


The Weyl character formula tells us that in order to compute the character of a finitedimensional, highest-weight irreducible representation of a complex semi-simple Lie algebra $\mathfrak{g}$, we should $(i)$ compute the product over the positive roots $\prod_{\alpha \in \Phi_{+}} \frac{1}{1-e^{-\alpha}}$, and then $(i i)$ apply the whole Weyl group to the highest-weight $\lambda$ shifted by the Weyl vector $\rho$. In the orthonormal basis $\mathrm{e}_{k}$ (with $k=0, \ldots, r$ ) of $\mathbb{R}^{r+1}$, the set of positive roots of $s o(2+d)$ is given by

- When $d=2 r$,

$$
\Phi_{+}=\left\{\mathrm{e}_{i} \pm \mathrm{e}_{j} \quad \text { with } \quad 0 \leqslant i<j \leqslant r\right\}
$$

- When $d=2 r+1$,

$$
\Phi_{+}=\left\{\mathrm{e}_{i} \pm \mathrm{e}_{j} \quad \text { with } \quad 0 \leqslant i<j \leqslant r\right\} \cup\left\{\mathrm{e}_{k} \quad \text { with } \quad k=0, \ldots, r\right\} .
$$

In the orthonormal basis, the components $\rho_{k}$ of the Weyl vector read:

$$
\rho_{k}=\frac{d}{2}-k, \quad k=0,1, \ldots, r
$$

Notice that we have shifted the components numbering on purpose, so that all the object defined above which do not have a 0th component can be reinterpreted as the same objects for the $s o(d)$ subalgebra. In other words, the components $\rho_{a}$ for $a=1, \ldots, r$ are those of the Weyl vector of $s o(d)$, and the positive roots previously enumerated which do not involve the unit vector $\mathrm{e}_{0}$ make up the positive root system of $s o(d)$ that we will denote $\Phi_{+}^{s o(d)}$.

Using (B.5) and (B.6), we can express the Weyl denominator of (B.1) for $s o(2+d)$ in terms of the Weyl denominator of $s o(d)$ as

$$
\mathrm{D}_{\mathrm{Weyl}}^{s o(2+d)}(\mu):=\prod_{\alpha \in \Phi_{+}} \frac{1}{1-e^{-(\alpha, \mu)}}
$$

In even dimensions, $d=2 r$, it becomes

$$
\begin{aligned}
\mathrm{D}_{\text {Weyl }}^{s o(2+2 r)}(\mu) & =\prod_{k=1}^{r} \frac{1}{\left(1-e^{-\left(\mathrm{e}_{0}, \mu\right)} e^{-\left(\mathrm{e}_{k}, \mu\right)}\right)\left(1-e^{-\left(\mathrm{e}_{0}, \mu\right)} e^{\left(\mathrm{e}_{k}, \mu\right)}\right)} \prod_{\alpha \in \Phi_{+}^{s o(2 r)}} \frac{1}{1-e^{-(\alpha, \mu)}} \\
& =\prod_{k=1}^{r} \frac{1}{\left(1-x_{0}^{-1} x_{k}^{-1}\right)\left(1-x_{0}^{-1} x_{k}\right)} \prod_{\alpha \in \Phi_{+}^{s o(2 r)}} \frac{1}{1-e^{-(\alpha, \mu)}} \\
& =\mathcal{P}_{2 r}\left(x_{0}^{-1}, \boldsymbol{x}\right) \mathrm{D}_{\mathrm{Weyl}}^{s o(2 r)}(\mu)
\end{aligned}
$$

where we defined the formal variables $x_{k}:=e^{\mu_{k}}$ for $k=0,1, \ldots, r$ and $\mathcal{P}_{2 r}\left(x_{0}, \boldsymbol{x}\right)$ is the function defined in (3.4). In odd dimensions, $d=2 r+1$, a similar computation - taking into account the additional root $\mathrm{e}_{0} \in \Phi_{+} \backslash \Phi_{+}^{s o(d)}$ with respect to the previous case - yields 
the same final result:

$$
\begin{aligned}
\mathrm{D}_{\mathrm{Weyl}}^{s o(3+2 r)}(\mu)= & \frac{1}{1-e^{-\left(\mathrm{e}_{0}, \mu\right)}} \prod_{k=1}^{r} \frac{1}{\left(1-e^{-\left(\mathrm{e}_{0}, \mu\right)} e^{-\left(\mathrm{e}_{k}, \mu\right)}\right)\left(1-e^{-\left(\mathrm{e}_{0}, \mu\right)} e^{\left(\mathrm{e}_{k}, \mu\right)}\right)} \\
\times & \prod_{\alpha \in \Phi_{+}^{s o(2 r+1)}} \frac{1}{1-e^{-(\alpha, \mu)}} \\
= & \mathcal{P}_{2 r+1}\left(x_{0}^{-1}, \boldsymbol{x}\right) \mathrm{D}_{\mathrm{Weyl}}^{s o(2 r+1)}(\mu) .
\end{aligned}
$$

Let us define

$$
\mathcal{C}_{\lambda}:=\frac{e^{\lambda}}{\prod_{\alpha \in \Phi_{+}}\left(1-e^{-\alpha}\right)},
$$

as well as the affine action of a Weyl element $w$ on a weight $\lambda$ :

$$
w \cdot \lambda:=w(\lambda+\rho)-\rho,
$$

where $w(\lambda)$ still denote the linear action of the Weyl element $w$ on the weight $\lambda$. Then we can rewrite the Weyl character formula as

$$
\chi_{\lambda}=\sum_{w \in \mathcal{W}} \varepsilon(w) \mathcal{C}_{w \cdot \lambda}
$$

It is furthermore possible to show that the following identity holds.

$$
\varepsilon(w) \mathcal{C}_{w \cdot \lambda}=w\left(\mathcal{C}_{\lambda}\right)
$$

and therefore (B.1) can be recasted as

$$
\chi_{\lambda}=\sum_{w \in \mathcal{W}} w\left(\mathcal{C}_{\lambda}\right)
$$

where the notation $w\left(\mathcal{C}_{\lambda}\right)$ represents the action of the reflection $w$ on the variables which the final character depends on, i.e. $\mu$. More concretely, in the case of $\mathfrak{g}=s o(2+d)$ of interest for us, the action of a generic element $w \in \mathcal{W}$ on a weight $\lambda$ is to first flip the sign of a number of components of $\lambda$ and then to permute those components. To each component of the weight $\mu$, we associated a formal variable, denoted above $x_{k}$ with $k=0, \ldots, r$ for the components of an $s o(2+d)$ weight, which carries this component as an exponent. As consequence, in the character formula the action of $w$ on a weight can be transfered as an operation on the variables $x_{k}$ : a sign flip of the $i$ th component of a weight can be equivalently represented as sending the corresponding variable $x_{i}$ to its inverse $x_{i}^{-1}$, and the permutation of several components, say the $i$ th and the $j$ th, of a weight are represented by the same permutation of the corresponding variables $x_{i}$ and $x_{j}$. With that in mind, we can simplify (B.1) by first summing on all elements of the Weyl group of $s o(d)$ (which we will denote $\left.\mathcal{W}_{s o(d)}\right)$, i.e. those reflections acting only on the last $r$ variables $x_{i}$ with $i=1, \ldots, r$. Using definitions (B.8) and (B.14) as well as formulae (B.11) and (B.13), we can write

$$
\mathcal{C}_{\lambda}^{s o(2+d)}\left(x_{0}, \boldsymbol{x}\right)=x_{0}^{\ell_{0}} \mathcal{C}_{\boldsymbol{\ell}}^{s o(d)}(\boldsymbol{x}) \mathcal{P}_{d}\left(x_{0}^{-1}, \boldsymbol{x}\right)
$$


where $\ell_{0}$ and $\ell \equiv\left(\ell_{1}, \ldots, \ell_{r}\right)$ are respectively the 0 th and last $r$ components of the $s o(2+d)$ highest weight $\lambda=\left(\ell_{0}, \ell_{1}, \ldots, \ell_{r}\right)$. Considering that the function $\mathcal{P}_{d}\left(x_{0}^{-1}, \boldsymbol{x}\right)$ is invariant under any $s o(d)$ Weyl group element (it is unchanged under any permutation or inversion of the variables $x_{i}$ with $\left.i=1, \ldots, r\right)$, acting with all elements of $\mathcal{W}_{s o(d)}$ on $\mathcal{C}_{\lambda}^{s o(2+d)}\left(x_{0}, \boldsymbol{x}\right)$ will produce the character of the irreducible $s o(d)$ representation with highest weight $\boldsymbol{\ell}$ out of the factor $\mathcal{C}_{\boldsymbol{\ell}}^{s o(d)}(\boldsymbol{x})$ :

$$
\sum_{w \in \mathcal{W}_{s o(d)}} w\left(\mathcal{C}_{\lambda}^{s o(2+d)}\left(x_{0}, \boldsymbol{x}\right)\right)=x_{0}^{\ell_{0}} \chi_{\boldsymbol{\ell}}^{s o(d)}(\boldsymbol{x}) \mathcal{P}_{d}\left(x_{0}^{-1}, \boldsymbol{x}\right)
$$

After having accounted for elements of the subgroup $\mathcal{W}_{s o(d)}$ of $\mathcal{W}$, the character formula reads:

$$
\chi_{\lambda}^{s o(2+d)}\left(x_{0}, \boldsymbol{x}\right)=\sum_{w \in \mathcal{W} \backslash \mathcal{W}_{s o(d)}} w\left(x_{0}^{\ell_{0}} \chi_{\ell}^{s o(d)}(\boldsymbol{x}) \mathcal{P}_{d}\left(x_{0}^{-1}, \boldsymbol{x}\right)\right) .
$$

Hence we need to take into account the elements of the Weyl group of $s o(2+d)$ that are not part of the subgroup $\mathcal{W}_{s o(d)}$, i.e. inversions of $x_{0}$ and permutations between $x_{0}$ and one of the other variables $x_{k}$ for $k=1, \ldots, r$. Using (3.6), the character can finally be put into the same form as (3.8):

$$
\chi_{\lambda}^{s o(2+d)}\left(x_{0}, \boldsymbol{x}\right)=\sum_{k=0}^{r}\left(x_{k}^{-\ell_{0}} \chi_{\boldsymbol{\ell}_{-}}^{s o(d)}\left(\boldsymbol{x}_{k}\right)+(-)^{d} x_{k}^{\ell_{0}+d} \chi_{\boldsymbol{\ell}_{+}}^{s o(d)}\left(\boldsymbol{x}_{k}\right)\right) \mathcal{P}_{d}\left(x_{k}, \boldsymbol{x}_{k}\right),
$$

with

$$
\ell_{ \pm} \equiv\left(\ell_{1}, \ldots, \ell_{r-1}, \pm \ell_{r}\right)
$$

for $d=2 r$ and $\boldsymbol{\ell}_{ \pm}=\boldsymbol{\ell}$ for $d=2 r+1$. Indeed, remember that the Weyl group for orthogonal algebras is a semi-direct product of the group of sign flips with the group of permutations, which is why (B.22) is composed a sum of two terms in which a variables $x_{k}$ is singled out: those two terms correspond to the two possibilities for $w \in \mathcal{W} \backslash \mathcal{W}_{s o(d)}$, either to invert $x_{0}$ or not. The relative factor of $\left(-x_{k}\right)^{d}$ between those two terms comes from the fact that the function $\mathcal{P}_{d}\left(x_{k}, \boldsymbol{x}_{k}\right)$ obey (3.6)

$$
\mathcal{P}_{d}\left(x_{k}^{-1}, \boldsymbol{x}_{k}\right)=\left(-x_{k}\right)^{d} \mathcal{P}_{d}\left(x_{k}, \boldsymbol{x}_{k}\right) .
$$

Finally, the change of chirality from $\ell_{+}$to $\ell_{-}$in even dimensions is due to the fact that in this case, any elements of the Weyl group has to be composed of an even number of sign flip of the components of the weights. This means that if the 0th component is sent to minus itself (equivalently, $x_{0}$ is inverted), then another of the $r$ remaining components has to also be affected. As noticed above, the $\mathcal{P}_{d}(q, \boldsymbol{x})$ function is invariant under any inversion of the variables $\boldsymbol{x}$, however one can show that

$$
\chi_{\ell_{+}}^{s o(2 r)}\left(x_{1}, \ldots, x_{k}^{-1}, \ldots, x_{r}\right)=\chi_{\ell_{-}}^{s o(2 r)}\left(x_{1}, \ldots, x_{k}, \ldots, x_{r}\right),
$$

i.e. inverting only one of the variables of the character of an $s o(2 r)$ irreducible representation produces the character of the $s o(2 r)$ irreducible representation with opposite chirality, ${ }^{17}$ which explains formula (B.22).

\footnotetext{
${ }^{17}$ Notice that this does not contradict the fact that the character of any irreducible representation of a compact Lie algebra $\mathfrak{g}$ is invariant under its Weyl group, as in the case of $s o(2 r)$ an element flipping the sign of an odd number of components of the highest weight is not part of the Weyl group $\mathcal{W}_{\text {so }(2 r)}$.
} 
Open Access. This article is distributed under the terms of the Creative Commons Attribution License (CC-BY 4.0), which permits any use, distribution and reproduction in any medium, provided the original author(s) and source are credited.

\section{References}

[1] P.A.M. Dirac, A Remarkable representation of the $3+2$ de Sitter group, J. Math. Phys. 4 (1963) 901 [INSPIRE].

[2] M. Flato and C. Fronsdal, One Massless Particle Equals Two Dirac Singletons: Elementary Particles in a Curved Space. 6., Lett. Math. Phys. 2 (1978) 421 [INSPIRE].

[3] M. Günaydin and N. Marcus, The Spectrum of the $S^{5}$ Compactification of the Chiral $N=2, D=10$ Supergravity and the Unitary Supermultiplets of $\mathrm{U}(2,2 / 4)$, Class. Quant. Grav. 2 (1985) L11 [INSPIRE].

[4] M. Günaydin and N. Marcus, The Unitary Supermultiplet of $N=8$ Conformal Superalgebra Involving Fields of Spin $\leq 2$, Class. Quant. Grav. 2 (1985) L19 [INSPIRE].

[5] M. Günaydin, P. van Nieuwenhuizen and N.P. Warner, General Construction of the Unitary Representations of Anti-de Sitter Superalgebras and the Spectrum of the $S^{4}$ Compactification of Eleven-dimensional Supergravity, Nucl. Phys. B 255 (1985) 63 [InSPIRE].

[6] M. Günaydin and N.P. Warner, Unitary Supermultiplets of Osp $(8 / 4, r)$ and the Spectrum of the $S_{7}$ Compactification of Eleven-dimensional Supergravity, Nucl. Phys. B 272 (1986) 99 [INSPIRE].

[7] E. Angelopoulos and M. Laoues, Singletons on AdS $S_{n}$, in Conference Moshe Flato Dijon, France, September 5-8, 1999, pp. 3-23, Mathematical Physics Studies, vol. 21/22, Springer (2000).

[8] M.A. Vasiliev, Higher spin superalgebras in any dimension and their representations, JHEP 12 (2004) 046 [hep-th/0404124] [INSPIRE].

[9] X. Bekaert and M. Grigoriev, Higher order singletons, partially massless fields and their boundary values in the ambient approach, Nucl. Phys. B 876 (2013) 667 [arXiv: 1305.0162] [INSPIRE].

[10] T. Basile, X. Bekaert and N. Boulanger, Flato-Fronsdal theorem for higher-order singletons, JHEP 11 (2014) 131 [arXiv:1410.7668] [InSPIRE].

[11] F.A. Dolan, Character formulae and partition functions in higher dimensional conformal field theory, J. Math. Phys. 47 (2006) 062303 [hep-th/0508031] [INSPIRE].

[12] E.S. Fradkin and M.A. Vasiliev, Candidate to the Role of Higher Spin Symmetry, Annals Phys. 177 (1987) 63 [INSPIRE].

[13] M.A. Vasiliev, Consistent equation for interacting gauge fields of all spins in (3+1)-dimensions, Phys. Lett. B 243 (1990) 378 [INSPIRE].

[14] X. Bekaert, S. Cnockaert, C. Iazeolla and M.A. Vasiliev, Nonlinear higher spin theories in various dimensions, in Higher spin gauge theories: Proceedings, 1st Solvay Workshop, Brussels, Belgium, 12-14 May 2004, pp. 132-197 [hep-th/0503128] [INSPIRE].

[15] V.E. Didenko and E.D. Skvortsov, Elements of Vasiliev theory, arXiv:1401.2975 [INSPIRE].

[16] M.G. Eastwood, Higher symmetries of the Laplacian, Annals Math. 161 (2005) 1645 [hep-th/0206233] [INSPIRE]. 
[17] M.A. Vasiliev, Nonlinear equations for symmetric massless higher spin fields in $(A) d S(d)$, Phys. Lett. B 567 (2003) 139 [hep-th/0304049] [INSPIRE].

[18] C. Iazeolla and P. Sundell, A Fiber Approach to Harmonic Analysis of Unfolded Higher-Spin Field Equations, JHEP 10 (2008) 022 [arXiv:0806.1942] [INSPIRE].

[19] R.K. Gupta and S. Lal, Partition Functions for Higher-Spin theories in AdS, JHEP 07 (2012) 071 [arXiv: 1205.1130] [INSPIRE].

[20] S. Lal, CFT(4) Partition Functions and the Heat Kernel on AdS(5), Phys. Lett. B 727 (2013) 325 [arXiv:1212.1050] [INSPIRE].

[21] S. Giombi, I.R. Klebanov and A.A. Tseytlin, Partition Functions and Casimir Energies in Higher Spin $A d S_{d+1} / C F T_{d}$, Phys. Rev. D 90 (2014) 024048 [arXiv:1402.5396] [InSPIRE].

[22] S. Giombi, I.R. Klebanov and B.R. Safdi, Higher Spin $A d S_{d+1} / C F T_{d}$ at One Loop, Phys. Rev. D 89 (2014) 084004 [arXiv: 1401.0825] [INSPIRE].

[23] A. Campoleoni, H.A. Gonzalez, B. Oblak and M. Riegler, Rotating Higher Spin Partition Functions and Extended BMS Symmetries, JHEP 04 (2016) 034 [arXiv:1512.03353] [INSPIRE].

[24] J.-B. Bae, E. Joung and S. Lal, On the Holography of Free Yang-Mills, JHEP 10 (2016) 074 [arXiv: 1607.07651] [INSPIRE].

[25] J.-B. Bae, E. Joung and S. Lal, One-loop test of free $\mathrm{SU}(N)$ adjoint model holography, JHEP 04 (2016) 061 [arXiv: 1603.05387] [INSPIRE].

[26] M. Günaydin, E.D. Skvortsov and T. Tran, Exceptional F(4) higher-spin theory in $A d S_{6}$ at one-loop and other tests of duality, JHEP 11 (2016) 168 [arXiv:1608.07582] [INSPIRE].

[27] J.-B. Bae, E. Joung and S. Lal, Exploring Free Matrix CFT Holographies at One-Loop, Universe 3 (2017) 77 [arXiv:1708.04644] [InSPIRE].

[28] M. Beccaria, X. Bekaert and A.A. Tseytlin, Partition function of free conformal higher spin theory, JHEP 08 (2014) 113 [arXiv:1406.3542] [INSPIRE].

[29] T. Basile, X. Bekaert and N. Boulanger, Mixed-symmetry fields in de Sitter space: a group theoretical glance, JHEP 05 (2017) 081 [arXiv:1612.08166] [INSPIRE].

[30] A. Bourget and J. Troost, The Conformal Characters, JHEP 04 (2018) 055 [arXiv: 1712.05415] [INSPIRE].

[31] M.A. Vasiliev, More on equations of motion for interacting massless fields of all spins in (3+1)-dimensions, Phys. Lett. B 285 (1992) 225 [inSPIRE].

[32] M.A. Vasiliev, Higher spin gauge theories: Star product and AdS space, hep-th/9910096 [INSPIRE].

[33] M.A. Vasiliev, Progress in higher spin gauge theories, in Quantization, gauge theory and strings. Proceedings, International Conference dedicated to the memory of Professor Efim Fradkin, Moscow, Russia, June 5-10, 2000. Vol. 1+2, pp. 452-471 (2001)

[DOI:10.1142/9789812777386_0199] [hep-th/0104246] [INSPIRE].

[34] J. Engquist, E. Sezgin and P. Sundell, On $N=1, N=2, N=4$ higher spin gauge theories in four-dimensions, Class. Quant. Grav. 19 (2002) 6175 [hep-th/0207101] [INSPIRE].

[35] E. Sezgin and P. Sundell, Geometry and Observables in Vasiliev's Higher Spin Gravity, JHEP 07 (2012) 121 [arXiv:1103.2360] [inSPIRE]. 
[36] I.R. Klebanov and A.M. Polyakov, AdS dual of the critical $O(N)$ vector model, Phys. Lett. B 550 (2002) 213 [hep-th/0210114] [INSPIRE].

[37] E. Sezgin and P. Sundell, Massless higher spins and holography, Nucl. Phys. B 644 (2002) 303 [Erratum ibid. B 660 (2003) 403] [hep-th/0205131] [INSPIRE].

[38] A. Joseph, The minimal orbit in a simple Lie algebra and its associated maximal ideal, Ann. Sci. École Norm. Sup. 9 (1976) 1.

[39] S. Fernando and M. Günaydin, Massless conformal fields, Ad $S_{d+1} / C F T_{d}$ higher spin algebras and their deformations, Nucl. Phys. B 904 (2016) 494 [arXiv:1511.02167] [INSPIRE].

[40] E. Joung and K. Mkrtchyan, Notes on higher-spin algebras: minimal representations and structure constants, JHEP 05 (2014) 103 [arXiv:1401.7977] [INSPIRE].

[41] M. Günaydin, Oscillator like unitary representations of noncompact groups and supergroups and extended supergravity theories, in Group Theoretical Methods in Physics. Proceedings, 11th International Colloquium, Istanbul, Turkey, August 23-28, 1982, pp. 192-213 (1983) [INSPIRE].

[42] M. Günaydin, Oscillator-Like Unitary Representations Of Non-Compact Groups And Supergroups And Extended Supergravity Theories, Lect. Notes Phys. 180 (1983) 192 [INSPIRE].

[43] S.E. Konshtein and M.A. Vasiliev, Massless Representations and Admissibility Condition for Higher Spin Superalgebras, Nucl. Phys. B 312 (1989) 402 [INSPIRE].

[44] R.G. Leigh and A.C. Petkou, Holography of the $N=1$ higher spin theory on AdS $S_{4}$,JHEP 06 (2003) 011 [hep-th/0304217] [INSPIRE].

[45] E. Sezgin and P. Sundell, Holography in $4 D$ (super) higher spin theories and a test via cubic scalar couplings, JHEP 07 (2005) 044 [hep-th/0305040] [INSPIRE].

[46] S.E. Konstein and M.A. Vasiliev, Extended Higher Spin Superalgebras and Their Massless Representations, Nucl. Phys. B 331 (1990) 475 [InSPIRE].

[47] E. Sezgin and P. Sundell, Doubletons and 5-D higher spin gauge theory, JHEP 09 (2001) 036 [hep-th/0105001] [INSPIRE].

[48] O.V. Shaynkman, I.Yu. Tipunin and M.A. Vasiliev, Unfolded form of conformal equations in $M$ dimensions and $o(M+2)$ modules, Rev. Math. Phys. 18 (2006) 823 [hep-th/0401086] [INSPIRE].

[49] R. Estrada and R. Kanwal, A Distributional Approach to Asymptotics: Theory and Applications, Birkhäuser Advanced Texts Basler Lehrbücher, Birkhäuser Boston (2002).

[50] K. Knopp, Theory and Application of Infinite Series, Dover Books on Mathematics, Dover Publications (1951).

[51] R.C. King, Modification rules and products of irreducible representations of the unitary, orthogonal and symplectic groups, J. Math. Phys. 12 (1971) 1588 [INSPIRE].

[52] X. Bekaert and N. Boulanger, The Unitary representations of the Poincaré group in any spacetime dimension, in 2nd Modave Summer School in Theoretical Physics Modave, Belgium, August 6-12, 2006 [hep-th/0611263] [INSPIRE].

[53] S. Giombi, I.R. Klebanov and Z.M. Tan, The ABC of Higher-Spin AdS/CFT, Universe 4 (2018) 18 [arXiv:1608.07611] [INSPIRE]. 
[54] E. Sezgin and P. Sundell, Supersymmetric Higher Spin Theories, J. Phys. A 46 (2013) 214022 [arXiv: 1208.6019] [INSPIRE].

[55] Y. Pang, E. Sezgin and Y. Zhu, One Loop Tests of Supersymmetric Higher Spin $A d S_{4} / C F T_{3}$, Phys. Rev. D 95 (2017) 026008 [arXiv: 1608.07298] [inSPIRE].

[56] P.S. Howe and U. Lindström, Super-Laplacians and their symmetries, JHEP 05 (2017) 119 [arXiv: 1612.06787] [INSPIRE].

[57] M. Günaydin, Singleton and doubleton supermultiplets of space-time supergroups and infinite spin superalgebras, in Trieste Conference on Supermembranes and Physics in $2+1$ Dimensions, Trieste, Italy, July 17-21, 1989, pp. 0442-456 [CERN-TH-5500-89].

[58] K. Govil and M. Günaydin, Deformed Twistors and Higher Spin Conformal (Super-)Algebras in Four Dimensions, JHEP 03 (2015) 026 [arXiv:1312.2907] [INSPIRE].

[59] K. Govil and M. Günaydin, Deformed Twistors and Higher Spin Conformal (Super-)Algebras in Six Dimensions, JHEP 07 (2014) 004 [arXiv:1401.6930] [INSPIRE].

[60] S. Fernando and M. Günaydin, Minimal unitary representation of $5 d$ superconformal algebra $F(4)$ and $A d S_{6} / C F T_{5}$ higher spin (super)-algebras, Nucl. Phys. B 890 (2014) 570 [arXiv: 1409.2185] [INSPIRE].

[61] K.B. Alkalaev, Global and local properties of AdS 2 higher spin gravity, JHEP 10 (2014) 122 [arXiv:1404.5330] [INSPIRE].

[62] D. Grumiller, M. Leston and D. Vassilevich, Anti-de Sitter holography for gravity and higher spin theories in two dimensions, Phys. Rev. D 89 (2014) 044001 [arXiv:1311.7413] [INSPIRE].

[63] K.B. Alkalaev, On higher spin extension of the Jackiw-Teitelboim gravity model, J. Phys. A 47 (2014) 365401 [arXiv: 1311.5119] [INSPIRE].

[64] A. Barut and C. Fronsdal, On non-compact groups. II. Representations of the $2+1$ Lorentz group, Proc. Roy. Soc. Lond. A 287 (1965) 532.

[65] A.U. Klimyk and N.Y. Vilenkin, Representations of Lie Groups and Special Functions, Springer Berlin Heidelberg (1995).

[66] A. Kitaev, Notes on $\widetilde{\mathrm{SL}}(2, \mathbb{R})$ representations, arXiv:1711.08169 [INSPIRE].

[67] J. Repka, Tensor products of unitary representations of $S L_{2}(R), A m$. J. Math. 100 (1978) 747.

[68] A. Joseph, Kostant's problem, Goldie rank and the Gelfand-Kirillov conjecture, Invent. Math. 56 (1980) 191.

[69] E.S. Fradkin and V.Ya. Linetsky, Infinite dimensional generalizations of finite dimensional symmetries, J. Math. Phys. 32 (1991) 1218 [inSPIRE].

[70] E.S. Fradkin and V.Ya. Linetsky, Infinite dimensional generalizations of simple Lie algebras, Mod. Phys. Lett. A 5 (1990) 1967 [INSPIRE].

[71] E. Hewitt and A. Kirillov, Elements of the Theory of Representations, Grundlehren der mathematischen Wissenschaften, Springer Berlin Heidelberg (2012).

[72] J. Dixmier, Enveloping algebras, vol. 14, Newnes (1977).

[73] B.L. Feigin, The Lie algebras $\mathfrak{g l}(\lambda)$ and cohomologies of Lie algebras of differential operators, Russ. Math. Surv. 43 (1988) 169. 
[74] N. Boulanger, D. Ponomarev, E. Sezgin and P. Sundell, New unfolded higher spin systems in $A d S_{3}$, Class. Quant. Grav. 32 (2015) 155002 [arXiv: 1412.8209] [INSPIRE].

[75] M.R. Gaberdiel, R. Gopakumar and A. Saha, Quantum $W$-symmetry in $A d S_{3}$, JHEP 02 (2011) 004 [arXiv: 1009.6087] [inSPIRE].

[76] M.R. Gaberdiel and R. Gopakumar, An AdS $S_{3}$ Dual for Minimal Model CFTs, Phys. Rev. D 83 (2011) 066007 [arXiv:1011.2986] [INSPIRE].

[77] M.R. Gaberdiel and R. Gopakumar, Minimal Model Holography, J. Phys. A 46 (2013) 214002 [arXiv: 1207.6697] [INSPIRE].

[78] E. Bergshoeff, M.P. Blencowe and K.S. Stelle, Area Preserving Diffeomorphisms and Higher Spin Algebra, Commun. Math. Phys. 128 (1990) 213 [inSPIRE].

[79] M. Bordemann, J. Hoppe and P. Schaller, Infinite dimensional matrix algebras, Phys. Lett. B 232 (1989) 199 [INSPIRE].

[80] M.A. Vasiliev, Higher Spin Algebras and Quantization on the Sphere and Hyperboloid, Int. J. Mod. Phys. A 6 (1991) 1115 [inSPIRE].

[81] S.F. Prokushkin and M.A. Vasiliev, Higher spin gauge interactions for massive matter fields in 3 -D AdS space-time, Nucl. Phys. B 545 (1999) 385 [hep-th/9806236] [InSPIRE].

[82] A. Campoleoni, S. Fredenhagen, S. Pfenninger and S. Theisen, Asymptotic symmetries of three-dimensional gravity coupled to higher-spin fields, JHEP 11 (2010) 007 [arXiv: 1008.4744] [INSPIRE].

[83] A. Campoleoni, S. Fredenhagen and S. Pfenninger, Asymptotic W-symmetries in three-dimensional higher-spin gauge theories, JHEP 09 (2011) 113 [arXiv:1107.0290] [INSPIRE].

[84] M. Henneaux and S.-J. Rey, Nonlinear $W_{\infty}$ as Asymptotic Symmetry of Three-Dimensional Higher Spin Anti-de Sitter Gravity, JHEP 12 (2010) 007 [arXiv:1008.4579] [InSPIRE].

[85] M. Henneaux, G. Lucena Gómez, J. Park and S.-J. Rey, Super-W $W_{\infty}$ Asymptotic Symmetry of Higher-Spin $A d S_{3}$ Supergravity, JHEP 06 (2012) 037 [arXiv:1203.5152] [INSPIRE].

[86] E.D. Skvortsov and M.A. Vasiliev, Geometric formulation for partially massless fields, Nucl. Phys. B 756 (2006) 117 [hep-th/0601095] [INSPIRE].

[87] K.B. Alkalaev, M. Grigoriev and E.D. Skvortsov, Uniformizing higher-spin equations, J. Phys. A 48 (2015) 015401 [arXiv:1409.6507] [inSPIRE].

[88] C. Brust and K. Hinterbichler, Partially Massless Higher-Spin Theory, JHEP 02 (2017) 086 [arXiv:1610.08510] [INSPIRE].

[89] C. Brust and K. Hinterbichler, Partially Massless Higher-Spin Theory II: One-Loop Effective Actions, JHEP 01 (2017) 126 [arXiv:1610.08522] [INSPIRE].

[90] E. Joung and K. Mkrtchyan, Partially-massless higher-spin algebras and their finite-dimensional truncations, JHEP 01 (2016) 003 [arXiv:1508.07332] [INSPIRE].

[91] M. Eastwood and T. Leistner, Higher symmetries of the square of the Laplacian, in Symmetries and overdetermined systems of partial differential equations, pp. 319-338. Springer (2008) [math/0610610].

[92] A.R. Gover and J. Šilhan, Higher symmetries of the conformal powers of the Laplacian on conformally flat manifolds, J. Math. Phys. 53 (2012) 032301 [arXiv:0911.5265]. 
[93] J.-P. Michel, Higher symmetries of the Laplacian via quantization, Annales Inst. Fourier 64 (2014) 1581 [arXiv: 1107.5840].

[94] K. Alkalaev, Mixed-symmetry tensor conserved currents and AdS/CFT correspondence, J. Phys. A 46 (2013) 214007 [arXiv:1207.1079] [InSPIRE].

[95] N. Boulanger, C. Iazeolla and P. Sundell, Unfolding Mixed-Symmetry Fields in AdS and the BMV Conjecture: I. General Formalism, JHEP 07 (2009) 013 [arXiv:0812.3615] [INSPIRE].

[96] N. Boulanger, C. Iazeolla and P. Sundell, Unfolding Mixed-Symmetry Fields in AdS and the BMV Conjecture. II. Oscillator Realization, JHEP 07 (2009) 014 [arXiv:0812.4438] [INSPIRE].

[97] E.D. Skvortsov, Gauge fields in $(A) d S_{d}$ and Connections of its symmetry algebra, J. Phys. A 42 (2009) 385401 [arXiv:0904.2919] [INSPIRE].

[98] E.D. Skvortsov, Gauge fields in $(A) d S_{d}$ within the unfolded approach: algebraic aspects, JHEP 01 (2010) 106 [arXiv:0910.3334] [INSPIRE].

[99] K. Alkalaev, Massless hook field in AdS $S_{d+1}$ from the holographic perspective, JHEP 01 (2013) 018 [arXiv: 1210.0217] [INSPIRE].

[100] W. Siegel, All Free Conformal Representations in All Dimensions, Int. J. Mod. Phys. A 4 (1989) 2015 [INSPIRE].

[101] E. Angelopoulos and M. Laoues, Masslessness in n-dimensions, Rev. Math. Phys. 10 (1998) 271 [hep-th/9806100] [INSPIRE].

[102] J.B. Ehrman, On the unitary irreducible representations of the universal covering group of the $3+2$ deSitter group, Math. Proc. Cambridge Phil. Soc. 53 (1957) 290.

[103] X. Bekaert, Singletons and their maximal symmetry algebras, in Modern Mathematical Physics. Proceedings, 6th Summer School, Belgrade, Serbia, September 14-23, 2010, pp. 71-89 (2011) [arXiv:1111.4554] [INSPIRE].

[104] N. Boulanger and E.D. Skvortsov, Higher-spin algebras and cubic interactions for simple mixed-symmetry fields in AdS spacetime, JHEP 09 (2011) 063 [arXiv:1107.5028] [INSPIRE].

[105] R. Manvelyan, K. Mkrtchyan, R. Mkrtchyan and S. Theisen, On Higher Spin Symmetries in $A d S_{5}, J H E P 10$ (2013) 185 [arXiv:1304.7988] [INSPIRE]. 